\title{
A chemical kinetic modeling study of indene pyrolysis
}

\section{Hanfeng Jin ${ }^{1}$, Lili Xing ${ }^{2 *}$, Junyu Hao ${ }^{1}$, Jiuzhong Yang ${ }^{3}$, Yan Zhang ${ }^{4}$, ChuangChuang Cao ${ }^{4}$, Yang}

$\operatorname{Pan}^{3}$, Aamir Farooq ${ }^{1 *}$

\footnotetext{
${ }^{I}$ Clean Combustion Research Centre, King Abdullah University of Science and Technology, Thuwal 23955-6900, Saudi Arabia

${ }^{2}$ Energy and Power Engineering Institute, Henan University of Science and Technology, Luoyang, Henan 471003, China

${ }^{3}$ National Synchrotron Radiation Laboratory, University of Science and Technology of China, Hefei, Anhui 230029, China

${ }^{4}$ Key Laboratory for Power Machinery and Engineering of MOE, Shanghai Jiao Tong University, Shanghai 200240, China
}

\section{Abstract}

An improved polycyclic aromatic hydrocarbon (PAH) model is developed to predict the decomposition of indene and the formation of large PAHs under pyrolytic conditions. This model is developed based on experimental study of pyrolytic kinetics of indene in a flow reactor at low and atmospheric pressures (30 and 760 Torr) by using synchrotron vacuum ultraviolet photoionization mass spectrometry (SVUV-PIMS). A general map of PAH growth is presented according to the observations in this study and those in literature. Indene dissociates via indanyl forming mono-cyclic aromatics and small intermediates, while its dominant decomposition product is indenyl. As a resonantly stabilized radical, indenyl serves as a platform molecule in PAH growth process which links small unsaturated hydrocarbons and mono-aromatic species to multi-cyclic ones. Reactions of indenyl radical are proposed to form commonly studied and recently observed PAHs. Rate constants of these reactions are evaluated by analyzing literature data of rate constant measurements, quantum chemical calculations and analogy to cyclopentadienyl radical. The main PAH formation pathways are the bimolecular addition reactions of indenyl radical with indene and a series of intermediates, forming $\mathrm{C}_{10^{-}}$ $\mathrm{C}_{18}$ and larger PAHs. Meanwhile, radical chain reactions provide huge passage for PAH growth form

*Corresponding authors. E-mail: aamir.farooq@kaust.edu.sa (AF), xing112018@haust.edu.cn (LX), Tel: +966128082704 (AF), +86-379-64231480 (LX). 
24 one resonantly stabilized radical (RSR) to larger ones. Particular contribution has been found from the

25 reactions of RSRs that have five-member ring in their molecular structures, such as fluorenyl, benz-

26 indenyl, cyclopenta-phenanthrenyl and benzo-fluorenyl.

27 Keywords: Indene mechanism; Flow reactor pyrolysis; SVUV-PIMS; Polycyclic aromatic hydrocarbons; 28 Resonantly stabilized radical.

29 


\section{Introduction}

Polycyclic aromatic hydrocarbons (PAHs) and soot are formed as a result of incomplete combustion, and contribute to particulate matter (PM) in our atmosphere. Human exposure to high density of PM enhances the chances of heart and lung diseases $[1,2]$. The investigation of the formation of PAHs and soot has, therefore, gained increasing attention since 1990s [3-6]. Various PAH models have been developed with a large array of possible reactions [7-15]. Herein, we briefly review the reaction kinetics for bicyclic aromatic species in these models.

blanks in the giant map of PAH species. Wang et al. [16] proposed hydrogen-abstraction-carbonaddition (HACA) mechanism in 1994, which was later supported by a number of experimental and theoretical investigations [13,17-20]. Models developed by Wang et al. [14] and Appel et al. [13] were then reported to describe the formation and growth of PAHs in flames fueled by $\mathrm{C}_{2}$ hydrocarbons, such as ethane, ethylene and acetylene. These models focused on HACA routes, and had relatively less consideration on toluene, indene, methylnaphthalene, etc., which may have high quantities in flames [15,21-23]. Later, Marinov et al. [12] extended the PAH model to cyclopenta[ $c d]$ pyrene, a $\mathrm{C}_{18} \mathrm{PAH}$, and Richter et al. [11] described the PAH growth up to coronene. They even proposed reaction pathways to form $\mathrm{C}_{60}-\mathrm{C}_{70}$ fullerene. These two works represented early investigations that not only included HACA mechanism but also the contributions from radical combination routes [12,24-29]. Propargyl and cyclopentadienyl radicals were then considered to be efficient contributors to the

49 formation of first and second aromatic ring, respectively [4,15,30-38]. Slavinskaya et al. [10,39] reviewed previous investigations and developed a PAH model up to five aromatic rings. Their model was validated against literature experimental data and provided semi-detailed chemical information for the PAH formation pathways. Later, Shulka and Koshi [40] added phenyl and methyl addition reactions in their model to extended the HACA mechanism. Hansen et al. [41] proposed repetitive

54 addition of methyl and acetylene for PAH growth. A series of radical-involved PAH formation pathways have been proposed and calculated [42-54]. Finally, a number of PAH models [7-9,19,30,55- 
58] have thus been developed and validated. We will not detail those studies here but will instead

57 discuss some recent works, particularly those closely related on indene.

A recent study of Johansson et al. [59] has conformed the critical role of resonantly stabilized radicals (RSR) in the growth of PAH and soot. Some recent theoretical calculations also revealed details of the reaction mechanisms of RSRs combination [47,48]. All these kinetic developments provide a good opportunity to model the PAH growth process from bicyclic aromatics to very large ones. Indene $\left(\mathrm{C}_{9} \mathrm{H}_{8}\right)$ is a typical bicyclic aromatic species, which is an important transmitting platform in the aromatic growth sequence. It could produce indenyl $\left(\mathrm{C}_{9} \mathrm{H}_{7}\right)$ radical via $\mathrm{H}$-abstraction or $\mathrm{H}$ elimination reactions. Indenyl radical has a resonantly stabilized molecular structure, and is, therefore, very participative in radical-radical and radical-neutral recombination reactions [59,60]. Small molecules can form indene or indenyl radical though multiple pathways, and then grow to large PAHs. Kinetic modeling study on indene can help reveal not only indenyl-involved PAH growth pathways but also the aggregation of small molecules formed by the decomposition of indene.

There are only a few literature studies related to indene chemical kinetics [61-67]. Badger and Kimber [67] performed gas chromatography (GC) measurements of indene pyrolysis, and observed significant amount of benzofluorenes, benz[a]anthracene, benzo-phenanthrene and chrysene. Laskin and Lifshitz [62] measured thermal decomposition products of indene behind reflected shock waves member ring dissociation of indanyl radical, while the decomposition of indenyl formed 1-ethynylcyclopenta-2,4-dienide $\left(\mathrm{C}_{7} \mathrm{H}_{5}\right)$ and cyclopentadienyl $\left(\mathrm{C}_{5} \mathrm{H}_{5}\right)$ radicals. Flash pyrolysis of 2-ethynyltoluene $\left(\mathrm{C}_{2} \mathrm{HC}_{6} \mathrm{H}_{4} \mathrm{CH}_{3}\right)$ was studied in the work of Ajaz et al. [61], and that of 3-methyl-4-(2'methyl)benzylideneisoxazol-5(4H)-one by Wentrup et al. [63]. The quantum chemical calculations of Wentrup et al. [63] explained the interconversion between indene and 2-ethynyl-toluene by RogerBrown rearrangement. Lifshitz et al. [66] investigated the pyrolysis of 2-methyl-indene in a shock tube, and described the mechanism of indenyl radical according to their experimental observations. 
82 Literature quantum chemical investigations of indene have focused on the addition reactions of indenyl.

83 The addition of acetylene on indenyl radical was calculated in the work of Cavallotti et al. [64]. In a

84 recent study, Mebel et al. [53] proposed the conversion from indene to naphthalene, emphasizing that

85 1-methyl-indene and 1-methyl-indenyl are critical intermediates in this process. The reaction between

86 indene and indenyl radical was investigated by $\mathrm{Lu}$ and Mulholland [65] via quantum chemical

87 calculations and species identification experiment. They proposed the product of this 9-step

88 mechanism to be chrysene, similar to the formation of naphthalene from cyclopentadienyl.

89 Calculations of Wentrup et al. [63] also showed that the combination of indenyl could produce bi-

90 indene and chrysene.

91 In the current work, detailed experimental data of indene pyrolysis in a flow reactor are reported

92 and used to propose a new model for indene kinetics. Experiments were performed with synchrotron

93 vacuum ultraviolet photoionization mass spectrometry (SVUV-PIMS) combined with molecular-beam

94 sampling technique [68,69]. Indene-contributed PAH sub-mechanism is constructed based on the

95 present experimental measurements and quantum chemical calculations. The major improvement in

96 the present model is the inclusion of semi-detailed PAH growth pathways via indenyl-involved

97 reactions. The model is validated against experimental data from this study and literature. Important

98 intermediates and reactions are identified for their critical role in the decomposition and ring expansion

99 of indene.

\section{Experimental details}

Indene pyrolysis was studied in a flow reactor at an undulator-based VUV beamline (BL03U) of

103 Hefei Light Source (HLS II), China. This new pyrolysis facility and the experimental evaluation

104 process have been introduced in detail in previous works [68,69]; only a brief description will be given

105 here. The flow reactor is composed of a home-made furnace and an $\alpha$-type alumina ceramic tube (inner

106 diameter $7.0 \mathrm{~mm}$, wall thickness $1.5 \mathrm{~mm}$ ). Electrically heated wire is uniformly twined in the inner

107 layer of the furnace, and the outermost layer acts as a heat shield. The total length of the heating zone 5 
108 is $\sim 220 \mathrm{~mm}$. A tungsten-rhenium (W-Re) thermocouple is positioned at the middle region to control

109 the reactor temperature. For the experiments reported here, mixtures of argon and indene were fed into

110 the alumina tube in the gas phase, and the pyrolysis species were sampled $10 \mathrm{~mm}$ downstream from

111 the outlet of the ceramic tube by a quartz nozzle. The forming molecular beam was passed through a

112 skimmer, and ionized by an orthogonal tunable VUV light. Ions were detected by a time-of-flight mass

113 spectrometer (TOF-MS) via an ion introducer.

114 Since temperature along the centerline of the flow reactor is a key parameter for the pyrolysis 115 process in the flow reactor, accurate temperature profiles were measured by an S-type thermocouple 116 before/after each measurement. Details about the temperature measurement are provided in SMM2 117 Section 1.1. The measured temperature profiles are given in SMM1, which are used in the simulation 118 of the flow reactor [70].

119 Liquid indene (purity 97\%) is injected in a vaporizer at $485 \mathrm{~K}$ before being fed into the flow 120 reactor. It is regulated by a high-pressure chromatography pump, while argon (purity $99.99 \%$ ) is 121 regulated by an MKS mass flow controller. The molar composition of the mixture is $0.32 \%$ indene / $12299.68 \%$ argon, and the total flow rate of the mixture is 1.5 SLM. Measurements of photoionization 123 efficiency (PIE) and mass spectra were carried out at two pressures, 30 and 760 Torr, and temperature 124 was varied in steps of $25 \mathrm{~K}$ over $975-1450 \mathrm{~K}$. The residence time is estimated to be 20 and $500 \mathrm{~ms}$ 125 at 30 and 760 torr, respectively. The uncertainty of SVUV-PIMS measurements comes primarily from 126 the sampling process and the photoionization cross-sections (PICS) of the measured species [68]. 127 Generally, the measurement uncertainty is $\pm 10 \%$ for species with direct PICS measurement, $\pm 25 \%$ for 128 stable species with PICS available in literature or database, and a factor of 2 for free radicals (usually 129 underestimated due to annihilation in the sampling process) and species with estimated PICS. Further 130 details of the experimental methodology, such as purity of indene, mole fraction evaluation, carbon 131 balance at each temperature, and PICSs of various species, are given in SMM2. 


\section{Theoretical calculations}

Quantum chemical calculations were employed in this work for the molecular structures, bond energies and reaction pathways. Geometry optimizations, electronic energies, and frequencies were computed using M08-HX method [71] with the MG3S basis set [72]. The MG3S basis set is identical to the $6-311+G(2 d f, 2 p)$ basis set for $H, C$, and O atoms [73]. Self-consistent field calculations and geometry optimization were performed with tight convergence criteria. Density functional integrations were carried out by a grid of 99 radial shells per atom with 974 angular points per shell. All calculations were performed using the Gaussian 16 software [74] with a self-developed MN-GFM6.7 module [75]. M08-HX/MG3S method was selected as an affordable electronic structure method because it has been shown to have uncertainty below $1 \mathrm{kcal} / \mathrm{mol}$ [76]. Cartesian coordinates (in $\AA$ ) and absolute energies of M08-HX/MG3S optimized geometries are collected in SMM3.

\section{Improved kinetic model}

We roughly divided indene model into two parts: the decomposition routes and the aromatic

147 growth routes. The first part of the current model is referred to the base models from Yuan et al. $[9,56]$,

148 Pousse et al. [77] and Lifshitz et al. [66]. The second part of the model is the PAH growth from indenyl 149 radical. The model of Yuan et al. is adopted as the base model, and then we built up reaction blocks

150 for large PAHs. We intended to keep the rate constants in the base model, and added necessary PAH 151 formation reactions driven by indenyl. Rate constants of these reactions are taken either from values 152 in the literature $[40,47,50,62,65,66,78]$ or analogized to similar reactions of cyclopentadienyl radical $153[46,48,51,64,79]$.

154 Table 1 Selected reactions in the present model

\begin{tabular}{|c|c|c|c|c|c|c|}
\hline No. & \multicolumn{2}{|l|}{ Reactions } & $\mathrm{A}^{\#}$ & $\mathrm{n}$ & $\mathrm{E}_{\mathrm{a}}^{\#}$ & Ref. \\
\hline 1 & \multicolumn{2}{|l|}{$\mathrm{C}_{9} \mathrm{H}_{7}+\mathrm{H}(+\mathrm{M})=\mathrm{C}_{9} \mathrm{H}_{8}(+\mathrm{M})$} & $1.10 \times 10^{14}$ & 0 & 0 & $\mathrm{a}$ \\
\hline & low & & $4.40 \times 10^{80}$ & -18.28 & 12994 & \\
\hline & troe & 0.068 & 400.7 & 4135.8 & 5501.9 & \\
\hline & \multicolumn{5}{|c|}{$\mathrm{H}_{2} / 2.0 / \mathrm{H}_{2} \mathrm{O} / 6.0 / \mathrm{CH}_{4} / 2.0 / \mathrm{CO} / 1.5 / \mathrm{CO}_{2} / 2.0 /$} & \\
\hline
\end{tabular}




\begin{tabular}{|c|c|c|c|c|c|c|}
\hline 2 & \multicolumn{2}{|l|}{$\mathrm{C}_{9} \mathrm{H}_{8}+\mathrm{H}=\mathrm{C}_{9} \mathrm{H}_{7}+\mathrm{H}_{2}$} & $2.22 \times 10^{15}$ & 1.874 & 4259 & $\mathrm{~b}$ \\
\hline 3 & \multicolumn{2}{|l|}{$\mathrm{C}_{9} \mathrm{H}_{8}+\mathrm{H}=\mathrm{C}_{9} \mathrm{H}_{9}{ }^{1}$} & $6.50 \times 10^{12}$ & 0 & 3100 & {$[79]$} \\
\hline 4 & \multicolumn{2}{|l|}{$\mathrm{C}_{9} \mathrm{H}_{8}+\mathrm{H}=\mathrm{C}_{9} \mathrm{H}_{9}^{2}$} & $5.50 \times 10^{12}$ & 0 & 7000 & {$[79]$} \\
\hline 5 & \multicolumn{2}{|l|}{$\mathrm{C}_{9} \mathrm{H}_{7}+\mathrm{CH}_{3}=\mathrm{C}_{9} \mathrm{H}_{7}^{1} \mathrm{CH}_{3}$} & $8.34 \times 10^{15}$ & -0.7 & -500 & {$[51]$} \\
\hline 6 & \multicolumn{2}{|l|}{$\mathrm{C}_{9} \mathrm{H}_{6}^{3} \mathrm{CH}_{3}=\mathrm{C}_{9} \mathrm{H}_{7}{ }^{1} \mathrm{CH}_{2}$} & $2.42 \times 10^{28}$ & 1 & 52470 & {$[78]$} \\
\hline 7 & \multicolumn{2}{|l|}{$\mathrm{C}_{9} \mathrm{H}_{7}{ }^{1} \mathrm{CH}_{2}=\mathrm{C}_{10} \mathrm{H}_{9}{ }^{1}$} & $2.42 \times 10^{28}$ & 1 & 12282 & {$[78]$} \\
\hline 8 & \multicolumn{2}{|l|}{$\mathrm{C}_{9} \mathrm{H}_{7}^{2} \mathrm{CH}_{2}=\mathrm{C}_{10} \mathrm{H}_{9}^{2}$} & $2.42 \times 10^{28}$ & 1 & 50520 & {$[78]$} \\
\hline 9 & \multicolumn{2}{|l|}{$\mathrm{C}_{10} \mathrm{H}_{9}{ }^{2}=\mathrm{C}_{10} \mathrm{H}_{9}{ }^{1}$} & $2.42 \times 10^{28}$ & 1 & 6970 & {$[78]$} \\
\hline 10 & \multicolumn{2}{|l|}{$\mathrm{C}_{10} \mathrm{H}_{9}{ }^{1}=\mathrm{bC}_{10} \mathrm{H}_{9}$} & $2.42 \times 10^{28}$ & 1 & 15120 & {$[78]$} \\
\hline 11 & \multicolumn{2}{|l|}{$\mathrm{C}_{9} \mathrm{H}_{7}{ }^{2} \mathrm{CH}_{2}=\mathrm{C}_{10} \mathrm{H}_{9}{ }^{3}$} & $2.42 \times 10^{28}$ & 1 & 53950 & {$[78]$} \\
\hline 12 & \multicolumn{2}{|l|}{$\mathrm{C}_{10} \mathrm{H}_{9}{ }^{3}=\mathrm{C}_{10} \mathrm{H}_{9}{ }^{4}$} & $2.42 \times 10^{28}$ & 1 & 21090 & {$[78]$} \\
\hline 13 & \multicolumn{2}{|l|}{$\mathrm{C}_{10} \mathrm{H}_{9}{ }^{4}=\mathrm{aC}_{10} \mathrm{H}_{9}$} & $2.42 \times 10^{28}$ & 1 & 42270 & {$[78]$} \\
\hline 14 & \multicolumn{2}{|l|}{$\mathrm{aC}_{10} \mathrm{H}_{9}=\mathrm{C}_{10} \mathrm{H}_{8}+\mathrm{H}$} & $2.42 \times 10^{28}$ & -5.83 & 23947 & {$[80]$} \\
\hline 15 & \multicolumn{2}{|l|}{$\mathrm{bC}_{10} \mathrm{H}_{9}=\mathrm{C}_{10} \mathrm{H}_{8}+\mathrm{H}$} & $2.42 \times 10^{28}$ & -5.83 & 23947 & {$[80]$} \\
\hline 16 & \multicolumn{2}{|l|}{$\mathrm{C}_{9} \mathrm{H}_{7}+\mathrm{CH}_{3}=\mathrm{C}_{9} \mathrm{H}_{6}{ }^{3} \mathrm{CH}_{3}+\mathrm{H}$} & & & & $\mathrm{c}$ \\
\hline & plog & 0.01 & $8.13 \times 10^{25}$ & -3.51 & 41018 & \\
\hline & plog & 1 & $3.83 \times 10^{26}$ & -3.69 & 41579 & \\
\hline & plog & 10 & $1.16 \times 10^{33}$ & -5.47 & 47110 & \\
\hline & plog & 100 & $1.03 \times 10^{48}$ & -9.54 & 61181 & \\
\hline 17 & \multicolumn{2}{|l|}{$\mathrm{C}_{9} \mathrm{H}_{7}{ }^{1} \mathrm{CH}_{3}+\mathrm{H}=\mathrm{C}_{9} \mathrm{H}_{8}+\mathrm{CH}_{3}$} & $8.00 \times 10^{13}$ & 0 & 10000 & {$[66]$} \\
\hline 18 & \multicolumn{2}{|l|}{$\mathrm{C}_{9} \mathrm{H}_{7}^{2} \mathrm{CH}_{3}+\mathrm{H}=\mathrm{C}_{9} \mathrm{H}_{8}+\mathrm{CH}_{3}$} & $8.00 \times 10^{13}$ & 0 & 10000 & {$[66]$} \\
\hline 19 & \multicolumn{2}{|l|}{$\mathrm{C}_{9} \mathrm{H}_{7}{ }^{3} \mathrm{CH}_{3}+\mathrm{H}=\mathrm{C}_{9} \mathrm{H}_{8}+\mathrm{CH}_{3}$} & $8.00 \times 10^{13}$ & 0 & 10000 & {$[66]$} \\
\hline 20 & \multicolumn{2}{|l|}{$\mathrm{C}_{9} \mathrm{H}_{7}+\mathrm{C}_{2} \mathrm{H}_{2}=\mathrm{C}_{9} \mathrm{H}_{7} \mathrm{C}_{2} \mathrm{H}+\mathrm{H}$} & & & & $\mathrm{d}$ \\
\hline & plog & 0.0001 & $5.58 \times 10^{11}$ & -0.1 & 34720 & \\
\hline & plog & 0.001 & $4.55 \times 10^{7}$ & 1.52 & 24370 & \\
\hline & plog & 0.01 & $5.75 \times 10^{6}$ & 1.79 & 24100 & \\
\hline & plog & 0.1 & $4.52 \times 10^{7}$ & 1.54 & 24710 & \\
\hline & plog & 1 & $8.17 \times 10^{11}$ & 0.33 & 27430 & \\
\hline & plog & 10 & $4.40 \times 10^{15}$ & -0.68 & 30890 & \\
\hline & plog & 100 & $7.24 \times 10^{15}$ & -0.61 & 34040 & \\
\hline & plog & 1000 & $1.88 \times 10^{7}$ & 2.07 & 34340 & \\
\hline \multirow[t]{7}{*}{21} & \multicolumn{2}{|l|}{$\mathrm{A}_{2}{ }^{1} \mathrm{CH}_{2}=>\mathrm{C}_{9} \mathrm{H}_{7} \mathrm{C}_{2} \mathrm{H}+\mathrm{H}$} & & & & $\mathrm{e}$ \\
\hline & plog & 0.013 & $4.47 \times 10^{129}$ & -32.57 & 162410 & \\
\hline & plog & 0.130 & $1.29 \times 10^{123}$ & -30.34 & 163830 & \\
\hline & plog & 1.000 & $2.95 \times 10^{97}$ & -22.95 & 148280 & \\
\hline & plog & 1.500 & $8.20 \times 10^{14}$ & 0 & 80700 & \\
\hline & plog & 10.00 & $1.26 \times 10^{39}$ & -6.76 & 99710 & \\
\hline & plog & 50.00 & $1.44 \times 10^{13}$ & 0.453 & 76300 & \\
\hline 22 & $\mathrm{C}_{9} \mathrm{H}_{7} \mathrm{C}_{2} \mathrm{H}+\mathrm{H}=>\mathrm{A}_{2}{ }^{1} \mathrm{CH}_{2}$ & & $8.80 \times 10^{8}$ & 1.2 & -2020 & $\mathrm{f}$ \\
\hline 23 & $\mathrm{~A}_{2}^{1} \mathrm{CH}_{2}=\mathrm{cC}_{11} \mathrm{H}_{9}$ & & & & & $\mathrm{~g}$ \\
\hline & plog & 0.013 & $1.12 \times 10^{128}$ & -32.57 & 162410 & \\
\hline & plog & 0.130 & $3.23 \times 10^{121}$ & -30.34 & 163830 & \\
\hline & plog & 1.000 & $7.38 \times 10^{95}$ & -22.95 & 148280 & \\
\hline & plog & 1.500 & $2.05 \times 10^{13}$ & 0 & 80700 & \\
\hline & plog & 10.00 & $3.15 \times 10^{37}$ & -6.76 & 99710 & \\
\hline & plog & 50.00 & $3.60 \times 10^{11}$ & 0.453 & 76300 & \\
\hline 24 & $\mathrm{cC}_{11} \mathrm{H}_{9}+\mathrm{H}=\mathrm{A}_{2}{ }^{1} \mathrm{CH}_{2}+\mathrm{H}$ & & $2.20 \times 10^{63}$ & -13 & 58628 & $\mathrm{~h}$ \\
\hline 25 & $\mathrm{cC}_{11} \mathrm{H}_{9}+\mathrm{H}=\mathrm{A}_{2^{-}}+\mathrm{CH}_{3}$ & & $1.20 \times 10^{61}$ & -12.4 & 57003 & $\mathrm{i}$ \\
\hline 26 & $\mathrm{C}_{9} \mathrm{H}_{7}+\mathrm{C}_{2} \mathrm{H}_{2}=\mathrm{cC}_{11} \mathrm{H}_{9}$ & & & & & $\mathrm{j}$ \\
\hline
\end{tabular}




\begin{tabular}{|c|c|c|c|c|c|c|}
\hline & plog & 0.0001 & $8.28 \times 10^{80}$ & -22.15 & 31790 & \\
\hline & plog & 0.0010 & $3.61 \times 10^{73}$ & -19.65 & 30230 & \\
\hline & plog & 0.0100 & $1.31 \times 10^{64}$ & -16.55 & 27790 & \\
\hline & plog & 0.1000 & $3.69 \times 10^{53}$ & -13.14 & 24810 & \\
\hline & plog & 1.0000 & $1.27 \times 10^{43}$ & -9.8 & 21720 & \\
\hline & plog & 10.000 & $1.07 \times 10^{34}$ & -6.9 & 19000 & \\
\hline & plog & 100.00 & $1.20 \times 10^{27}$ & -4.67 & 16940 & \\
\hline & plog & 1000.0 & $1.09 \times 10^{23}$ & -3.37 & 15770 & \\
\hline 27 & \multicolumn{2}{|l|}{$\mathrm{C}_{9} \mathrm{H}_{7}+\mathrm{C}_{3} \mathrm{H}_{3}=\mathrm{R}_{5} \mathrm{C}_{12} \mathrm{H}_{10}$} & $5.78 \times 10^{17}$ & -1.568 & 455 & $\mathrm{k}$ \\
\hline & dup & & $3.14 \times 10^{19}$ & -2.163 & 1195 & \\
\hline 28 & \multicolumn{2}{|l|}{$\mathrm{C}_{9} \mathrm{H}_{7}+\mathrm{C}_{3} \mathrm{H}_{3}=\mathrm{R}_{5} \mathrm{C}_{12} \mathrm{H}_{9}+\mathrm{H}$} & & & & 1 \\
\hline & plog & 0.04 & $1.05 \times 10^{53}$ & -11.88 & 28757 & \\
\hline & plog & 1.00 & $1.70 \times 10^{48}$ & -9.977 & 26755 & \\
\hline & plog & 10.0 & $3.67 \times 10^{26}$ & -3.879 & 28963 & \\
\hline \multirow[t]{4}{*}{29} & \multicolumn{2}{|c|}{$\mathrm{R}_{5} \mathrm{C}_{12} \mathrm{H}_{9}+\mathrm{H}(+\mathrm{M})=\mathrm{C}_{9} \mathrm{H}_{7} \mathrm{C}_{3} \mathrm{H}_{3}(+\mathrm{M})$} & $1.00 \times 10^{14}$ & 0 & 0 & $\mathrm{a}$ \\
\hline & low & & $4.40 \times 10^{80}$ & -18.28 & 12994 & \\
\hline & troe & 0.068 & 400.7 & 4135.8 & 5501.9 & \\
\hline & \multicolumn{5}{|c|}{$\mathrm{H}_{2} / 2.0 / \mathrm{H}_{2} \mathrm{O} / 6.0 / \mathrm{CH}_{4} / 2.0 / \mathrm{CO} / 1.5 / \mathrm{CO}_{2} / 2.0 /$} & \\
\hline 30 & \multicolumn{2}{|l|}{$\mathrm{R}_{5} \mathrm{C}_{12} \mathrm{H}_{10}+\mathrm{H}=\mathrm{R}_{5} \mathrm{C}_{12} \mathrm{H}_{9}+\mathrm{H}_{2}$} & $5.60 \times 10^{13}$ & 0 & 2259 & $\mathrm{~m}$ \\
\hline \multirow[t]{7}{*}{31} & \multicolumn{2}{|l|}{$\mathrm{R}_{5} \mathrm{C}_{12} \mathrm{H}_{9}=\mathrm{R}_{5} \mathrm{C}_{12} \mathrm{H}_{8}+\mathrm{H}$} & & & & $\mathrm{n}$ \\
\hline & plog & 0.00132 & $6.28 \times 10^{71}$ & -18.204 & 65711 & \\
\hline & plog & 0.0132 & $2.82 \times 10^{61}$ & -14.813 & 62790 & \\
\hline & plog & 0.132 & $3.77 \times 10^{47}$ & -10.443 & 58072 & \\
\hline & plog & 1 & $7.54 \times 10^{33}$ & -6.226 & 52769 & \\
\hline & plog & 10 & $4.44 \times 10^{21}$ & -2.491 & 47796 & \\
\hline & plog & 100 & $2.86 \times 10^{13}$ & 0.015 & 44395 & \\
\hline \multirow[t]{7}{*}{32} & \multicolumn{2}{|l|}{$\mathrm{R}_{5} \mathrm{C}_{12} \mathrm{H}_{9}=\mathrm{A}_{2} \mathrm{R}_{5}+\mathrm{H}$} & & & & o \\
\hline & plog & 0.00132 & $3.68 \times 10^{63}$ & -15.806 & 57410 & \\
\hline & plog & 0.0132 & $6.88 \times 10^{51}$ & -12.098 & 53279 & \\
\hline & plog & 0.132 & $2.37 \times 10^{38}$ & -7.917 & 48317 & \\
\hline & plog & 1 & $9.04 \times 10^{25}$ & -4.123 & 43308 & \\
\hline & plog & 10 & $4.13 \times 10^{16}$ & -1.278 & 39451 & \\
\hline & plog & 100 & $5.55 \times 10^{12}$ & -0.065 & 38028 & \\
\hline \multirow[t]{7}{*}{33} & \multicolumn{2}{|l|}{$\mathrm{R}_{5} \mathrm{C}_{12} \mathrm{H}_{8}+\mathrm{H}=\mathrm{A}_{2} \mathrm{R}_{5}+\mathrm{H}$} & & & & $\mathrm{p}$ \\
\hline & plog & 0.00132 & $4.94 \times 10^{18}$ & -1.28 & 5411 & \\
\hline & plog & 0.0132 & $2.15 \times 10^{22}$ & -2.28 & 8429 & \\
\hline & plog & 0.132 & $5.60 \times 10^{26}$ & -3.47 & 12818 & \\
\hline & plog & 1 & $1.66 \times 10^{25}$ & -2.99 & 13691 & \\
\hline & plog & 1.312 & $5.06 \times 10^{25}$ & -3.12 & 14226 & \\
\hline & plog & 13.12 & $2.20 \times 10^{27}$ & -3.48 & 19199 & \\
\hline \multirow[t]{4}{*}{34} & \multicolumn{2}{|l|}{$\mathrm{R}_{5} \mathrm{C}_{12} \mathrm{H}_{8}=\mathrm{A}_{2} \mathrm{R}_{5}$} & & & & $\mathrm{p}$ \\
\hline & plog & 0.04 & $5.62 \times 10^{81}$ & -19.36 & 121500 & \\
\hline & plog & 1.00 & $1.45 \times 10^{45}$ & -8.90 & 95999 & \\
\hline & plog & 10.0 & $2.95 \times 10^{31}$ & -4.97 & 88465 & \\
\hline \multirow[t]{4}{*}{35} & \multicolumn{2}{|l|}{$\mathrm{C}_{9} \mathrm{H}_{7}+\mathrm{C}_{3} \mathrm{H}_{3}=\mathrm{A}_{2} \mathrm{C}_{2} \mathrm{H}_{3}$} & & & & $\mathrm{q}$ \\
\hline & plog & 0.04 & $1.82 \times 10^{73}$ & -18.14 & 31896 & \\
\hline & plog & 1.00 & $3.16 \times 10^{54}$ & -12.55 & 22264 & \\
\hline & plog & 10.0 & $3.88 \times 10^{49}$ & -11.01 & 20320 & \\
\hline 36 & $\mathrm{C}_{9} \mathrm{H}_{7}+\mathrm{C}_{5} \mathrm{H}_{6}=\mathrm{C}_{9} \mathrm{H}_{7} \mathrm{C}_{5} \mathrm{H}_{6}$ & & $1.00 \times 10^{-4}$ & 4.2 & 3400 & $\mathrm{r}$ \\
\hline
\end{tabular}




\begin{tabular}{|c|c|c|c|c|c|c|}
\hline 37 & \multicolumn{2}{|l|}{$\mathrm{C}_{9} \mathrm{H}_{8}+\mathrm{C}_{5} \mathrm{H}_{5}=\mathrm{C}_{9} \mathrm{H}_{8} \mathrm{C}_{5} \mathrm{H}_{5}$} & $1.00 \times 10^{-4}$ & 4.2 & 3400 & $\mathrm{r}$ \\
\hline 38 & \multicolumn{2}{|l|}{$\mathrm{C}_{9} \mathrm{H}_{7} \mathrm{C}_{5} \mathrm{H}_{6}=\mathrm{fC}_{13} \mathrm{H}_{10} \mathrm{CH}_{3}$} & $7.00 \times 10^{9}$ & 1.17 & 48500 & $\mathrm{~s}$ \\
\hline 39 & \multicolumn{2}{|l|}{$\mathrm{C}_{9} \mathrm{H}_{7} \mathrm{C}_{5} \mathrm{H}_{6}=\mathrm{bC}_{13} \mathrm{H}_{10} \mathrm{CH}_{3}$} & $7.00 \times 10^{9}$ & 1.17 & 48500 & $\mathrm{~s}$ \\
\hline 40 & \multicolumn{2}{|l|}{$\mathrm{C}_{9} \mathrm{H}_{8} \mathrm{C}_{5} \mathrm{H}_{5}=\mathrm{fC}_{13} \mathrm{H}_{10} \mathrm{CH}_{3}$} & $7.00 \times 10^{9}$ & 1.17 & 48500 & $\mathrm{~s}$ \\
\hline 41 & \multicolumn{2}{|l|}{$\mathrm{C}_{9} \mathrm{H}_{8} \mathrm{C}_{5} \mathrm{H}_{5}=\mathrm{bC}_{13} \mathrm{H}_{10} \mathrm{CH}_{3}$} & $7.00 \times 10^{9}$ & 1.17 & 48500 & $\mathrm{~s}$ \\
\hline 42 & \multicolumn{2}{|l|}{$\mathrm{fC}_{13} \mathrm{H}_{10} \mathrm{CH}_{3}=\mathrm{C}_{13} \mathrm{H}_{10}+\mathrm{CH}_{3}$} & $1.52 \times 10^{13}$ & 0.396 & 32820 & $\mathrm{t}$ \\
\hline 43 & \multicolumn{2}{|l|}{$\mathrm{bC}_{13} \mathrm{H}_{10} \mathrm{CH}_{3}=\mathrm{bC}_{13} \mathrm{H}_{10}+\mathrm{CH}_{3}$} & $1.52 \times 10^{13}$ & 0.396 & 32820 & $\mathrm{t}$ \\
\hline 44 & \multicolumn{2}{|l|}{$\mathrm{A}_{2}{ }^{1} \mathrm{CH}_{2}+\mathrm{C}_{2} \mathrm{H}_{2}=\mathrm{bC}_{13} \mathrm{H}_{10}+\mathrm{H}$} & $3.12 \times 10^{-6}$ & 4.712 & 1417.5 & $\mathrm{u}$ \\
\hline 45 & \multicolumn{2}{|l|}{$\mathrm{A}_{2}+\mathrm{C}_{3} \mathrm{H}_{3}=\mathrm{bC}_{13} \mathrm{H}_{10}+\mathrm{H}$} & $6.26 \times 10^{9}$ & 2.61 & 56500 & $\mathrm{v}$ \\
\hline 46 & \multicolumn{2}{|l|}{$\mathrm{C}_{9} \mathrm{H}_{7}+\mathrm{C}_{5} \mathrm{H}_{5}=\mathrm{C}_{9} \mathrm{H}_{7} \mathrm{C}_{5} \mathrm{H}_{5}$} & & & & $\mathrm{w}$ \\
\hline & plog & 0.04 & $3.14 \times 10^{174}$ & -49.43 & 62602 & \\
\hline & plog & 1.00 & $1.80 \times 10^{188}$ & -52.62 & 76380 & \\
\hline & plog & 10.0 & $4.28 \times 10^{174}$ & -47.91 & 75125 & \\
\hline 47 & \multicolumn{2}{|l|}{$\mathrm{C}_{9} \mathrm{H}_{7}+\mathrm{C}_{5} \mathrm{H}_{5}=\mathrm{C}_{9} \mathrm{H}_{6} \mathrm{C}_{5} \mathrm{H}_{5}+\mathrm{H}$} & $5.60 \times 10^{13}$ & 0 & 2259 & $\mathrm{x}$ \\
\hline 48 & \multicolumn{2}{|c|}{$\mathrm{C}_{9} \mathrm{H}_{6} \mathrm{C}_{5} \mathrm{H}_{5}+\mathrm{H}(+\mathrm{M})=\mathrm{C}_{9} \mathrm{H}_{7} \mathrm{C}_{5} \mathrm{H}_{5}(+\mathrm{M})$} & $1.00 \times 10^{14}$ & 0 & 0 & $\mathrm{a}$ \\
\hline & low & & $4.40 \times 10^{80}$ & -18.28 & 12994 & \\
\hline & troe & 0.068 & 400.7 & 4135.8 & 5501.9 & \\
\hline & \multicolumn{5}{|c|}{$\mathrm{H}_{2} / 2.0 / \mathrm{H}_{2} \mathrm{O} / 6.0 / \mathrm{CH}_{4} / 2.0 / \mathrm{CO} / 1.5 / \mathrm{CO}_{2} / 2.0 /$} & \\
\hline 49 & \multicolumn{2}{|c|}{$\mathrm{C}_{9} \mathrm{H}_{7} \mathrm{C}_{5} \mathrm{H}_{5}+\mathrm{H}=\mathrm{C}_{9} \mathrm{H}_{6} \mathrm{C}_{5} \mathrm{H}_{5}+\mathrm{H}_{2}$} & $1.12 \times 10^{14}$ & 0 & 2259 & $\mathrm{~m}$ \\
\hline 50 & \multicolumn{2}{|l|}{$\mathrm{C}_{9} \mathrm{H}_{6} \mathrm{C}_{5} \mathrm{H}_{5}=\mathrm{A}_{3} \mathrm{H}$} & $5.60 \times 10^{8}$ & 1.31 & 19100 & $\mathrm{y}$ \\
\hline 51 & \multicolumn{2}{|l|}{$\mathrm{A}_{3} \mathrm{H}=\mathrm{A}_{3}+\mathrm{H}$} & $6.80 \times 10^{11}$ & 0.508 & 18659 & $\mathrm{z}$ \\
\hline 52 & \multicolumn{2}{|l|}{$\mathrm{C}_{9} \mathrm{H}_{7} \mathrm{C}_{5} \mathrm{H}_{6}=\mathrm{C}_{9} \mathrm{H}_{7} \mathrm{C}_{5} \mathrm{H}_{5}+\mathrm{H}$} & $1.55 \times 10^{10}$ & 1.16 & 45500 & aa \\
\hline 53 & \multicolumn{2}{|l|}{$\mathrm{C}_{9} \mathrm{H}_{8} \mathrm{C}_{5} \mathrm{H}_{5}=\mathrm{C}_{9} \mathrm{H}_{7} \mathrm{C}_{5} \mathrm{H}_{5}^{\mathrm{b}}+\mathrm{H}$} & $1.55 \times 10^{10}$ & 1.16 & 45500 & aa \\
\hline 54 & \multicolumn{2}{|l|}{$\mathrm{fC}_{13} \mathrm{H}_{9}+\mathrm{CH}_{3}=\mathrm{fC}_{13} \mathrm{H}_{9} \mathrm{CH}_{3}$} & $6.62 \times 10^{13}$ & -0.7 & -500 & $\mathrm{bb}$ \\
\hline 55 & \multicolumn{2}{|l|}{$\mathrm{bC}_{13} \mathrm{H}_{9}+\mathrm{CH}_{3}=\mathrm{bC}_{13} \mathrm{H}_{9} \mathrm{CH}_{3}$} & $6.62 \times 10^{13}$ & -0.7 & -500 & $\mathrm{bb}$ \\
\hline 56 & \multicolumn{2}{|l|}{$\mathrm{A}_{3} \mathrm{CH}_{2}=\mathrm{bC}_{13} \mathrm{H}_{9}+\mathrm{C}_{2} \mathrm{H}_{2}$} & & & & $\mathrm{cc}$ \\
\hline & plog & 0.013 & $4.08 \times 10^{134}$ & -34.08 & 169130 & \\
\hline & plog & 0.130 & $1.29 \times 10^{112}$ & -27.5 & 155360 & \\
\hline & plog & 1.000 & $1.10 \times 10^{89}$ & -20.82 & 141680 & \\
\hline & plog & 10.00 & $1.03 \times 10^{55}$ & -11.23 & 116580 & \\
\hline & plog & 50.00 & $1.44 \times 10^{13}$ & 0.453 & 76300 & \\
\hline 57 & $\mathrm{~A}_{3} \mathrm{CH}_{2}=\mathrm{cC}_{15} \mathrm{H}_{11}$ & & & & & $\mathrm{~g}$ \\
\hline & plog & 0.013 & $1.12 \times 10^{128}$ & -32.57 & 162410 & \\
\hline & plog & 0.13 & $3.23 \times 10^{121}$ & -30.34 & 163830 & \\
\hline & plog & 1 & $7.38 \times 10^{95}$ & -22.95 & 148280 & \\
\hline & plog & 1.5 & $2.05 \times 10^{13}$ & 0 & 80700 & \\
\hline & plog & 10 & $3.15 \times 10^{37}$ & -6.76 & 99710 & \\
\hline & plog & 50 & $3.60 \times 10^{12}$ & 0.453 & 76300 & \\
\hline 58 & $\mathrm{C}_{9} \mathrm{H}_{7}+\mathrm{C}_{6} \mathrm{H}_{5}=\mathrm{C}_{9} \mathrm{H}_{7} \mathrm{C}_{6} \mathrm{H}_{5}$ & & & & & $\mathrm{w}$ \\
\hline & plog & 0.013 & $3.14 \times 10^{174}$ & -49.43 & 62602 & \\
\hline & plog & 1.000 & $1.90 \times 10^{188}$ & -52.62 & 76380 & \\
\hline & plog & 100.0 & $4.30 \times 10^{174}$ & -47.91 & 75125 & \\
\hline 59 & $\mathrm{C}_{9} \mathrm{H}_{6} \mathrm{C}_{6} \mathrm{H}_{5}+\mathrm{H}(+\mathrm{M})=\mathrm{C}_{9} \mathrm{H}_{7}$ & & $1.00 \times 10^{14}$ & 0 & 0 & $\mathrm{a}$ \\
\hline & low & & $4.40 \times 10^{80}$ & -18.28 & 12994 & \\
\hline & troe & 0.068 & 400.7 & 4135.8 & 5501.9 & \\
\hline & $\mathrm{H}_{2} / 2$ & $.0 / \mathrm{CH}_{4}$ & $2.0 / \mathrm{CO} / 1.5 /$ & & & \\
\hline 60 & $\mathrm{C}_{9} \mathrm{H}_{7} \mathrm{C}_{6} \mathrm{H}_{5}+\mathrm{H}=\mathrm{C}_{9} \mathrm{H}_{6} \mathrm{C}_{6} \mathrm{H}_{5}$ & & $1.12 \times 10^{14}$ & 0 & 2259 & $\mathrm{~m}$ \\
\hline 61 & $\mathrm{C}_{9} \mathrm{H}_{6} \mathrm{C}_{6} \mathrm{H}_{5}=\mathrm{cC}_{15} \mathrm{H}_{11}$ & & $8.40 \times 10^{11}$ & 0.456 & 42368 & [47] \\
\hline 62 & $\mathrm{cC}_{15} \mathrm{H}_{11}=\mathrm{cpC}_{15} \mathrm{H}_{10}+\mathrm{H}$ & & $6.40 \times 10^{12}$ & 0 & 34800 & $\mathrm{dd}$ \\
\hline
\end{tabular}




\begin{tabular}{|c|c|c|c|c|c|c|}
\hline 63 & \multicolumn{2}{|l|}{$\mathrm{C}_{9} \mathrm{H}_{7}+\mathrm{A}_{1} \mathrm{CH}_{2}=\mathrm{C}_{9} \mathrm{H}_{7} \mathrm{C}_{7} \mathrm{H}_{7}$} & $8.10 \times 10^{11}$ & -0.369 & 1172 & [47] \\
\hline 64 & \multicolumn{2}{|l|}{$\mathrm{C}_{9} \mathrm{H}_{7} \mathrm{C}_{7} \mathrm{H}_{7}=\mathrm{C}_{9} \mathrm{H}_{7} \mathrm{CHC}_{6} \mathrm{H}_{5}+\mathrm{H}$} & $1.30 \times 10^{11}$ & 0.77 & 4354 & [47] \\
\hline 65 & \multicolumn{2}{|c|}{$\mathrm{C}_{9} \mathrm{H}_{7} \mathrm{C}_{7} \mathrm{H}_{7}+\mathrm{H}=\mathrm{C}_{9} \mathrm{H}_{7} \mathrm{CHC}_{6} \mathrm{H}_{5}+\mathrm{H}_{2}$} & 4.32 & 3.98 & 1380 & [47] \\
\hline 66 & \multicolumn{2}{|l|}{$\mathrm{C}_{9} \mathrm{H}_{7} \mathrm{CHC}_{6} \mathrm{H}_{5}=\mathrm{C}_{10} \mathrm{H}_{8} \mathrm{C}_{6} \mathrm{H}_{5}$} & $8.10 \times 10^{11}$ & 0.038 & 11810 & [47] \\
\hline 67 & \multicolumn{2}{|l|}{$\mathrm{C}_{10} \mathrm{H}_{7} \mathrm{C}_{6} \mathrm{H}_{5}+\mathrm{H}=\mathrm{C}_{10} \mathrm{H}_{8} \mathrm{C}_{6} \mathrm{H}_{5}$} & & & & ee \\
\hline & plog & 0.0263 & $1.16 \times 10^{41}$ & -9.51 & 10830 & \\
\hline & plog & 0.1184 & $2.40 \times 10^{40}$ & -9.06 & 11570 & \\
\hline & plog & 1.0000 & $6.82 \times 10^{35}$ & -7.37 & 11230 & \\
\hline \multirow[t]{4}{*}{68} & \multicolumn{2}{|c|}{$\mathrm{C}_{10} \mathrm{H}_{6} \mathrm{C}_{6} \mathrm{H}_{5}+\mathrm{H}(+\mathrm{M})=\mathrm{C}_{10} \mathrm{H}_{7} \mathrm{C}_{6} \mathrm{H}_{5}(+\mathrm{M})$} & $1.00 \times 10^{14}$ & 0 & 0 & $\mathrm{ff}$ \\
\hline & low & & $6.6 . \times 10^{75}$ & -16.3 & 7000 & \\
\hline & troe & 1 & 0.1 & 584.9 & 6113 & \\
\hline & \multicolumn{5}{|c|}{$\mathrm{H}_{2} / 2.0 / \mathrm{H}_{2} \mathrm{O} / 6.0 / \mathrm{CH}_{4} / 2.0 / \mathrm{CO} / 1.5 / \mathrm{CO}_{2} / 2.0 / \mathrm{C}_{2} \mathrm{H}_{6} / 3.0 /$} & \\
\hline 69 & \multicolumn{2}{|c|}{$\mathrm{C}_{10} \mathrm{H}_{7} \mathrm{C}_{6} \mathrm{H}_{5}+\mathrm{H}=\mathrm{C}_{10} \mathrm{H}_{6} \mathrm{C}_{6} \mathrm{H}_{5}+\mathrm{H}_{2}$} & $5.90 \times 10^{11}$ & 0.705 & 12626 & [47] \\
\hline 70 & \multicolumn{2}{|l|}{$\mathrm{C}_{10} \mathrm{H}_{6} \mathrm{C}_{6} \mathrm{H}_{5}=\mathrm{C}_{16} \mathrm{H}_{11}{ }^{\mathrm{f}}$} & $8.40 \times 10^{11}$ & 0.456 & 42368 & [47] \\
\hline 71 & \multicolumn{2}{|l|}{$\mathrm{C}_{16} \mathrm{H}_{11}^{\mathrm{f}}=\mathrm{C}_{16} \mathrm{H}_{11}^{\mathrm{p}}$} & $6.60 \times 10^{11}$ & 0.566 & 39778 & [47] \\
\hline 72 & \multicolumn{2}{|l|}{$\mathrm{C}_{16} \mathrm{H}_{11}{ }^{\mathrm{f}}=\mathrm{fC}_{16} \mathrm{H}_{10}+\mathrm{H}$} & $6.90 \times 10^{11}$ & 0.548 & 25832 & [47] \\
\hline 73 & \multicolumn{2}{|l|}{$\mathrm{C}_{16} \mathrm{H}_{11}^{\mathrm{p}}=\mathrm{pA}_{4}+\mathrm{H}$} & $6.80 \times 10^{11}$ & 0.508 & 18659 & [47] \\
\hline 74 & \multicolumn{2}{|l|}{$\mathrm{cpC}_{15} \mathrm{H}_{9}+\mathrm{CH}_{3}=\mathrm{cpC}_{15} \mathrm{H}_{9} \mathrm{CH}_{3}$} & $6.62 \times 10^{13}$ & -0.7 & -500 & $\mathrm{bb}$ \\
\hline 75 & \multicolumn{2}{|c|}{$\mathrm{cpC}_{15} \mathrm{H}_{9}+\mathrm{CH}_{3}=\mathrm{cpC}_{15} \mathrm{H}_{8} \mathrm{CH}_{3}+\mathrm{H}$} & & & & $\mathrm{c}$ \\
\hline & plog & 0.01 & $3.03 \times 10^{15}$ & -0.58 & 10871 & \\
\hline & plog & 0.10 & $1.23 \times 10^{31}$ & -1.4 & 24769 & \\
\hline & plog & 1.00 & $3.23 \times 10^{46}$ & -5.9 & 39791 & \\
\hline & plog & 10.0 & $1.49 \times 10^{56}$ & -9.05 & 52592 & \\
\hline 76 & \multicolumn{2}{|l|}{$\mathrm{cpC}_{15} \mathrm{H}_{9} \mathrm{CH}_{3}=\mathrm{cpC}_{15} \mathrm{H}_{8} \mathrm{CH}_{3}+\mathrm{H}$} & & & & gg \\
\hline & plog & 0.10 & $1.74 \times 10^{97}$ & -24.38 & 116128 & \\
\hline & plog & 1.00 & $5.77 \times 10^{97}$ & -24.0 & 122296 & \\
\hline & plog & 10.0 & $1.75 \times 10^{87}$ & -20.6 & 120401 & \\
\hline 77 & \multicolumn{2}{|c|}{$\mathrm{cpC}_{15} \mathrm{H}_{9} \mathrm{CH}_{3}+\mathrm{H}=\mathrm{cpC}_{15} \mathrm{H}_{8} \mathrm{CH}_{3}+\mathrm{H}_{2}$} & $1.30 \times 10^{6}$ & 2.38 & 2800 & [81] \\
\hline 78 & \multicolumn{2}{|l|}{$\mathrm{cpC}_{15} \mathrm{H}_{8} \mathrm{CH}_{3}=\mathrm{pA}_{4}+\mathrm{H}$} & $9.25 \times 10^{43}$ & -9.191 & 55757 & $\mathrm{hh}$ \\
\hline & dup & & $1.98 \times 10^{31}$ & -6.066 & 20630 & \\
\hline 79 & \multicolumn{2}{|l|}{$\mathrm{C}_{9} \mathrm{H}_{7}+\mathrm{C}_{9} \mathrm{H}_{8}=\mathrm{C}_{9} \mathrm{H}_{8} \mathrm{C}_{9} \mathrm{H}_{7}$} & $1.00 \times 10^{-4}$ & 4.2 & 3400 & $\mathrm{r}$ \\
\hline 80 & \multicolumn{2}{|l|}{$\mathrm{C}_{9} \mathrm{H}_{8} \mathrm{C}_{9} \mathrm{H}_{7}=\mathrm{C}_{17} \mathrm{H}_{12} \mathrm{CH}_{3}{ }^{\mathrm{b}}$} & $7.00 \times 10^{9}$ & 1.17 & 48500 & $\mathrm{~s}$ \\
\hline 81 & \multicolumn{2}{|l|}{$\mathrm{C}_{17} \mathrm{H}_{12} \mathrm{CH}_{3}{ }^{\mathrm{b}}=\mathrm{BCF}+\mathrm{CH}_{3}$} & $1.52 \times 10^{13}$ & 0.396 & 32820 & $\mathrm{t}$ \\
\hline 82 & $\mathrm{bC}_{13} \mathrm{H}_{9}+\mathrm{C}_{5} \mathrm{H}_{6}=\mathrm{bC}_{13} \mathrm{H}_{9} \mathrm{C}_{5} \mathrm{H}_{6}$ & & $1.00 \times 10^{-4}$ & 4.2 & 3400 & $\mathrm{r}$ \\
\hline 83 & $\mathrm{bC}_{13} \mathrm{H}_{9} \mathrm{C}_{5} \mathrm{H}_{6}=\mathrm{C}_{17} \mathrm{H}_{12} \mathrm{CH}_{3}{ }^{\mathrm{b}}$ & & $7.00 \times 10^{9}$ & 1.17 & 48500 & $\mathrm{~s}$ \\
\hline 84 & $\mathrm{bC}_{13} \mathrm{H}_{10}+\mathrm{C}_{5} \mathrm{H}_{5}=\mathrm{bC}_{13} \mathrm{H}_{10} \mathrm{C}_{5} \mathrm{H}$ & & $1.00 \times 10^{-4}$ & 4.2 & 3400 & $\mathrm{r}$ \\
\hline 85 & $\mathrm{bC}_{13} \mathrm{H}_{10} \mathrm{C}_{5} \mathrm{H}_{5}=\mathrm{C}_{17} \mathrm{H}_{12} \mathrm{CH}_{3}{ }^{\mathrm{b}}$ & & $7.00 \times 10^{9}$ & 1.17 & 48500 & $\mathrm{~s}$ \\
\hline 86 & $\mathrm{C}_{17} \mathrm{H}_{12} \mathrm{CH}_{3}{ }^{\mathrm{b}}=\mathrm{BCF}^{5} \mathrm{CH}_{3}+\mathrm{H}$ & & $6.80 \times 10^{11}$ & 0.508 & 18659 & $\mathrm{z}$ \\
\hline 87 & $\mathrm{BCF}-+\mathrm{CH}_{3}=\mathrm{BCF}^{11} \mathrm{CH}_{3}$ & & $6.62 \times 10^{13}$ & -0.7 & -500 & $\mathrm{bb}$ \\
\hline 88 & $\mathrm{C}_{9} \mathrm{H}_{7}+\mathrm{C}_{9} \mathrm{H}_{7}=>\mathrm{C}_{9} \mathrm{H}_{7} \mathrm{C}_{9} \mathrm{H}_{7}$ & & & & & $\mathrm{w}$ \\
\hline & plog & 0.0132 & $3.14 \times 10^{174}$ & -49.43 & 62602 & \\
\hline & plog & 1.0000 & $1.90 \times 10^{188}$ & -52.62 & 76380 & \\
\hline & plog & 100.00 & $4.30 \times 10^{174}$ & -47.91 & 75125 & \\
\hline 89 & $\mathrm{C}_{9} \mathrm{H}_{7} \mathrm{C}_{9} \mathrm{H}_{7} \Rightarrow \mathrm{C}_{9} \mathrm{H}_{7}+\mathrm{C}_{9} \mathrm{H}_{7}$ & & & & & $\mathrm{w}$ \\
\hline & plog & 0.0132 & $2.08 \times 10^{70}$ & -17.55 & 62254 & \\
\hline & plog & 1.0000 & $1.47 \times 10^{77}$ & -18.71 & 73651 & \\
\hline & plog & 100.00 & $1.24 \times 10^{64}$ & -14.25 & 72842 & \\
\hline 90 & $\mathrm{bC}_{13} \mathrm{H}_{9}+\mathrm{C}_{5} \mathrm{H}_{5}=\mathrm{bC}_{13} \mathrm{H}_{9} \mathrm{C}_{5} \mathrm{H}_{5}$ & & & & & $\mathrm{w}$ \\
\hline & plog & 0.0132 & $3.14 \times 10^{174}$ & -49.43 & 62602 & \\
\hline
\end{tabular}




\begin{tabular}{|c|c|c|c|c|c|c|}
\hline & plog & 1.0000 & $1.90 \times 10^{188}$ & -52.62 & 76380 & \\
\hline & plog & 100.00 & $4.30 \times 10^{174}$ & -47.91 & 75125 & \\
\hline 91 & \multicolumn{2}{|l|}{$\mathrm{fC}_{13} \mathrm{H}_{9}+\mathrm{C}_{5} \mathrm{H}_{5}=\mathrm{fC}_{13} \mathrm{H}_{9} \mathrm{C}_{5} \mathrm{H}_{5}$} & & & & $\mathrm{w}$ \\
\hline & plog & 0.0132 & $3.14 \times 10^{174}$ & -49.43 & 62602 & \\
\hline & plog & 1.0000 & $1.90 \times 10^{188}$ & -52.62 & 76380 & \\
\hline & plog & 100.00 & $4.30 \times 10^{174}$ & -47.91 & 75125 & \\
\hline 92 & \multicolumn{2}{|c|}{$\mathrm{C}_{9} \mathrm{H}_{6} \mathrm{C}_{9} \mathrm{H}_{7}+\mathrm{H}(+\mathrm{M})=\mathrm{C}_{9} \mathrm{H}_{7} \mathrm{C}_{9} \mathrm{H}_{7}(+\mathrm{M})$} & $1.00 \times 10^{14}$ & 0 & 0 & $\mathrm{a}$ \\
\hline & low & & $4.40 \times 10^{80}$ & -18.28 & 12994 & \\
\hline & troe & 0.068 & 400.7 & 4135.8 & 5501.9 & \\
\hline & \multicolumn{5}{|c|}{$\mathrm{H}_{2} / 2.0 / \mathrm{H}_{2} \mathrm{O} / 6.0 / \mathrm{CH}_{4} / 2.0 / \mathrm{CO} / 1.5 / \mathrm{CO}_{2} / 2.0 /$} & \\
\hline 93 & \multicolumn{2}{|c|}{$\mathrm{C}_{9} \mathrm{H}_{7} \mathrm{C}_{9} \mathrm{H}_{7}+\mathrm{H}=\mathrm{C}_{9} \mathrm{H}_{6} \mathrm{C}_{9} \mathrm{H}_{7}+\mathrm{H}_{2}$} & $1.12 \times 10^{14}$ & 0 & 2259 & [57] \\
\hline 94 & \multicolumn{2}{|c|}{$\mathrm{C}_{9} \mathrm{H}_{7} \mathrm{C}_{9} \mathrm{H}_{7}+\mathrm{C}_{9} \mathrm{H}_{7}=\mathrm{C}_{9} \mathrm{H}_{6} \mathrm{C}_{9} \mathrm{H}_{7}+\mathrm{C}_{9} \mathrm{H}_{8}$} & $3.20 \times 10^{11}$ & 0 & 15100 & ii \\
\hline 95 & \multicolumn{2}{|l|}{$\mathrm{C}_{9} \mathrm{H}_{6} \mathrm{C}_{9} \mathrm{H}_{7}=\mathrm{C}_{18} \mathrm{H}_{13}{ }^{\mathrm{c}}$} & $5.60 \times 10^{8}$ & 1.31 & 19100 & $\mathrm{y}$ \\
\hline 96 & \multicolumn{2}{|l|}{$\mathrm{C}_{9} \mathrm{H}_{6} \mathrm{C}_{9} \mathrm{H}_{7}=\mathrm{C}_{18} \mathrm{H}_{13}{ }^{\mathrm{p}}$} & $5.60 \times 10^{8}$ & 1.31 & 19100 & $\mathrm{y}$ \\
\hline 97 & \multicolumn{2}{|l|}{$\mathrm{C}_{18} \mathrm{H}_{13}{ }^{\mathrm{c}}=\mathrm{C}_{18} \mathrm{H}_{12}{ }^{\mathrm{c}}+\mathrm{H}$} & & & & $\mathrm{z}$ \\
\hline & plog & 0.04 & $3.40 \times 10^{10}$ & 0.78 & 30230 & \\
\hline & plog & 1.00 & $6.80 \times 10^{11}$ & 0.508 & 18659 & \\
\hline 98 & \multicolumn{2}{|l|}{$\mathrm{C}_{18} \mathrm{H}_{13}^{\mathrm{p}}=\mathrm{C}_{18} \mathrm{H}_{12}{ }^{\mathrm{p}}+\mathrm{H}$} & & & & $\mathrm{z}$ \\
\hline & plog & 0.04 & $3.40 \times 10^{10}$ & 0.78 & 30230 & \\
\hline & plog & 1.00 & $6.80 \times 10^{11}$ & 0.508 & 18659 & \\
\hline 99 & \multicolumn{2}{|l|}{$\mathrm{C}_{9} \mathrm{H}_{7}+\mathrm{C}_{9} \mathrm{H}_{7}=\mathrm{C}_{9} \mathrm{H}_{6} \mathrm{C}_{9} \mathrm{H}_{7}+\mathrm{H}$} & $5.25 \times 10^{14}$ & -0.853 & 7254 & $\mathrm{x}$ \\
\hline 100 & \multicolumn{2}{|c|}{$\mathrm{bC}_{13} \mathrm{H}_{8} \mathrm{C}_{5} \mathrm{H}_{5}+\mathrm{H}(+\mathrm{M})=\mathrm{bC}_{13} \mathrm{H}_{9} \mathrm{C}_{5} \mathrm{H}_{5}(+\mathrm{M})$} & $1.00 \times 10^{14}$ & 0 & 0 & $\mathrm{a}$ \\
\hline & low & & $4.40 \times 10^{80}$ & -18.28 & 12994 & \\
\hline & troe & 0.068 & 400.7 & 4135.8 & 5501.9 & \\
\hline & \multicolumn{5}{|c|}{$\mathrm{H}_{2} / 2.0 / \mathrm{H}_{2} \mathrm{O} / 6.0 / \mathrm{CH}_{4} / 2.0 / \mathrm{CO} / 1.5 / \mathrm{CO}_{2} / 2.0 /$} & \\
\hline 101 & \multicolumn{2}{|c|}{$\mathrm{bC}_{13} \mathrm{H}_{9} \mathrm{C}_{5} \mathrm{H}_{5}+\mathrm{H}=\mathrm{bC}_{13} \mathrm{H}_{8} \mathrm{C}_{5} \mathrm{H}_{5}+\mathrm{H}_{2}$} & $5.60 \times 10^{13}$ & 0 & 2259 & $\mathrm{~m}$ \\
\hline 102 & \multicolumn{2}{|c|}{$\mathrm{bC}_{13} \mathrm{H}_{8} \mathrm{C}_{5} \mathrm{H}_{5}=\mathrm{C}_{18} \mathrm{H}_{13}{ }^{\mathrm{c}}$} & $5.60 \times 10^{8}$ & 1.31 & 19100 & $\mathrm{y}$ \\
\hline 103 & \multicolumn{2}{|l|}{$\mathrm{bC}_{13} \mathrm{H}_{8} \mathrm{C}_{5} \mathrm{H}_{5}=\mathrm{C}_{18} \mathrm{H}_{13}{ }^{\mathrm{p}}$} & $5.60 \times 10^{8}$ & 1.31 & 19100 & $\mathrm{y}$ \\
\hline 104 & \multicolumn{2}{|c|}{$\mathrm{bC}_{13} \mathrm{H}_{9}+\mathrm{C}_{5} \mathrm{H}_{5}=\mathrm{bC}_{13} \mathrm{H}_{8} \mathrm{C}_{5} \mathrm{H}_{5}+\mathrm{H}$} & $5.25 \times 10^{14}$ & -0.853 & 7254 & $\mathrm{x}$ \\
\hline & dup & & $2.00 \times 10^{10}$ & 0.951 & 15793 & \\
\hline 105 & \multicolumn{2}{|c|}{$\mathrm{fC}_{13} \mathrm{H}_{8} \mathrm{C}_{5} \mathrm{H}_{5}+\mathrm{H}(+\mathrm{M})=\mathrm{fC}_{13} \mathrm{H}_{9} \mathrm{C}_{5} \mathrm{H}_{5}(+\mathrm{M})$} & $1.00 \times 10^{14}$ & 0 & 0 & $\mathrm{a}$ \\
\hline & low & & $4.40 \times 10^{80}$ & -18.28 & 12994 & \\
\hline & troe & 0.068 & 400.7 & 4135.8 & 5501.9 & \\
\hline & \multicolumn{5}{|c|}{$\mathrm{H}_{2} / 2.0 / \mathrm{H}_{2} \mathrm{O} / 6.0 / \mathrm{CH}_{4} / 2.0 / \mathrm{CO} / 1.5 / \mathrm{CO}_{2} / 2.0 /$} & \\
\hline 106 & \multicolumn{2}{|c|}{$\mathrm{fC}_{13} \mathrm{H}_{9} \mathrm{C}_{5} \mathrm{H}_{5}+\mathrm{H}=\mathrm{fC}_{13} \mathrm{H}_{8} \mathrm{C}_{5} \mathrm{H}_{5}+\mathrm{H}_{2}$} & $5.60 \times 10^{13}$ & 0 & 2259 & $\mathrm{~m}$ \\
\hline 107 & \multicolumn{2}{|l|}{$\mathrm{fC}_{13} \mathrm{H}_{8} \mathrm{C}_{5} \mathrm{H}_{5}=\mathrm{C}_{18} \mathrm{H}_{13}{ }^{\mathrm{t}}$} & $5.60 \times 10^{8}$ & 1.31 & 19100 & $\mathrm{y}$ \\
\hline 108 & \multicolumn{2}{|l|}{$\mathrm{C}_{18} \mathrm{H}_{13}{ }^{\mathrm{t}}=\mathrm{C}_{18} \mathrm{H}_{12}{ }^{\mathrm{t}}+\mathrm{H}$} & $6.80 \times 10^{11}$ & 0.508 & 18659 & $\mathrm{z}$ \\
\hline 109 & \multicolumn{2}{|c|}{$\mathrm{fC}_{13} \mathrm{H}_{9}+\mathrm{C}_{5} \mathrm{H}_{5}=\mathrm{fC}_{13} \mathrm{H}_{8} \mathrm{C}_{5} \mathrm{H}_{5}+\mathrm{H}$} & $5.25 \times 10^{14}$ & -0.853 & 7254 & $\mathrm{x}$ \\
\hline & dup & & $2.00 \times 10^{10}$ & 0.951 & 15793 & \\
\hline
\end{tabular}

\# The rate constants are given in the form of $k=A \cdot T^{n} \cdot e^{-E_{a} / R T}$, where A has the units of $\mathrm{cm}$, mol and $\mathrm{s}$; $\mathrm{T}$ has the unit of $\mathrm{K} ; \mathrm{E}_{\mathrm{a}}$ has the unit of $\mathrm{cal} / \mathrm{mol}$.

157 a Rate constant analogized to $\mathrm{C}_{5} \mathrm{H}_{5}+\mathrm{H}=\mathrm{C}_{5} \mathrm{H}_{6}$ in JetSurf 2.0 model [82].

158 b Rate constant analogized to $\mathrm{C}_{5} \mathrm{H}_{6}+\mathrm{H}=\mathrm{C}_{5} \mathrm{H}_{5}+\mathrm{H}_{2}$, calculated by Robinson et al. [34].

c Rate constant analogized to $\mathrm{C}_{5} \mathrm{H}_{5}+\mathrm{CH}_{3}=\mathrm{C}_{5} \mathrm{H}_{5} \mathrm{CH}_{2}+\mathrm{H}$, calculated by Sharma and Green [51].

$160 \mathrm{~d}$ Rate constant analogized to $\mathrm{C}_{5} \mathrm{H}_{5}+\mathrm{C}_{2} \mathrm{H}_{2}=\mathrm{C}_{7} \mathrm{H}_{6}+\mathrm{H}$ calculated by Da Silva et al. [83].

161 e Rate constant analogized to $\mathrm{A}_{1} \mathrm{CH}_{2}=\mathrm{C}_{7} \mathrm{H}_{6}+\mathrm{H}$ calculated by Polino et al. [84].

$162 \mathrm{f}$ Rate constant analogized to $\mathrm{C}_{7} \mathrm{H}_{6}+\mathrm{H}=\mathrm{A}_{1} \mathrm{CH}_{2}$ calculated by Cavallotti et al. [85].

$163 \mathrm{~g}$ Rate constant analogized to $\mathrm{A}_{1} \mathrm{CH}_{2}=\mathrm{cC}_{7} \mathrm{H}_{7}$ estimated in the model of Yuan et al. [56].

$164 \mathrm{~h}$ Rate constant analogized to $\mathrm{cC}_{7} \mathrm{H}_{7}+\mathrm{H}=\mathrm{A}_{1} \mathrm{CH}_{2}+\mathrm{H}$ calculated by Fascella et al. [79].

165 i Rate constant analogized to $\mathrm{cC}_{7} \mathrm{H}_{7}+\mathrm{H}=\mathrm{A}_{1^{-}}+\mathrm{CH}_{3}$ calculated by Fascella et al. [79].

$166 \mathrm{j}$ Rate constant analogized to $\mathrm{C}_{5} \mathrm{H}_{5}+\mathrm{C}_{2} \mathrm{H}_{2}=\mathrm{cC}_{7} \mathrm{H}_{7}$ calculated by Da Silva et al. [83].

167 k Rate constant taken equal to $\mathrm{A}_{1} \mathrm{CH}_{2}+\mathrm{C}_{3} \mathrm{H}_{3}=\mathrm{A}_{1} \mathrm{CH}_{2} \mathrm{C}_{3} \mathrm{H}_{3}$ calculated by Matsugi and Miyoshi [45]. 
1 Rate constant taken equal to $\mathrm{A}_{1} \mathrm{CH}_{2}+\mathrm{C}_{3} \mathrm{H}_{3}=\mathrm{C}_{10} \mathrm{H}_{9}+\mathrm{H}$ in the model of Matsugi and Miyoshi [57]. m Rate constant analogized to $\mathrm{C}_{5} \mathrm{H}_{5} \mathrm{C}_{5} \mathrm{H}_{5}+\mathrm{H}=\mathrm{C}_{5} \mathrm{H}_{5} \mathrm{C}_{5} \mathrm{H}_{4}+\mathrm{H}_{2}$ calculated Matsugi and Miyoshi [57].

$n$ Rate constant taken equal to $\mathrm{C}_{9} \mathrm{H}_{6} \mathrm{CH}_{3}=\mathrm{C}_{9} \mathrm{H}_{6} \mathrm{CH}_{2}+\mathrm{H}$ calculated by Matsugi and Miyoshi [45].

o Rate constant taken equal to $\mathrm{C}_{9} \mathrm{H}_{6} \mathrm{CH}_{3}=\mathrm{A}_{2}+\mathrm{H}$ calculated by Matsugi and Miyoshi [45].

$\mathrm{p}$ Rate constant taken equal to the isomerization of $\mathrm{C}_{9} \mathrm{H}_{6} \mathrm{CH}_{2}$ and $\mathrm{A}_{2}$ calculated by Matsugi and Miyoshi [45].

q Rate constant analogized to $\mathrm{C}_{5} \mathrm{H}_{5}+\mathrm{C}_{3} \mathrm{H}_{3}=\mathrm{A}_{1} \mathrm{C}_{2} \mathrm{H}_{3}$ in the model of Yuan et al. [42,55].

$r$ Rate constant analogized to $\mathrm{C}_{5} \mathrm{H}_{5}+\mathrm{C}_{5} \mathrm{H}_{6}=\mathrm{C}_{5} \mathrm{H}_{6} \mathrm{C}_{5} \mathrm{H}_{5}$ calculated by Kislov et al. [86].

s Rate constant taken equal to the controlling step of the isomerization from $\mathrm{C}_{5} \mathrm{H}_{6} \mathrm{C}_{5} \mathrm{H}_{5}$ to $\mathrm{C}_{9} \mathrm{H}_{6} \mathrm{CH}_{3}$, calculated by Kislov et al. [86].

t Rate constant taken equal to $\mathrm{C}_{6} \mathrm{H}_{10} \mathrm{CH}_{3}=\mathrm{C}_{6} \mathrm{H}_{10}+\mathrm{CH}_{3}$ in the model of Narayanaswamy et al. [87].

$\mathrm{u}$ Rate constant analogized to $\mathrm{A}_{1} \mathrm{CH}_{2}+\mathrm{C}_{2} \mathrm{H}_{2}=\mathrm{C}_{9} \mathrm{H}_{8}+\mathrm{H}$ by the calculation of Vereecken and Peeters [88].

$\mathrm{v}$ Product of this reaction changes to benzo-indene, it is proposed to form fluorene in the model of Yuan et al. [56].

w Rate constant analogized to $\mathrm{C}_{5} \mathrm{H}_{5}+\mathrm{C}_{5} \mathrm{H}_{5}=\mathrm{C}_{5} \mathrm{H}_{5} \mathrm{C}_{5} \mathrm{H}_{5}$ calculated by Matsugi and Miyoshi [57].

$x$ Rate constant analogized to $\mathrm{C}_{5} \mathrm{H}_{5}+\mathrm{C}_{5} \mathrm{H}_{5}=\mathrm{C}_{5} \mathrm{H}_{5} \mathrm{C}_{5} \mathrm{H}_{4}+\mathrm{H}$ calculated by Cavallotti et al. [46].

y Rate constant analogized to $\mathrm{C}_{5} \mathrm{H}_{5} \mathrm{C}_{5} \mathrm{H}_{4}=\mathrm{C}_{10} \mathrm{H}_{9}$ calculated by Kislov et al. [43].

z Rate constant analogized to $\mathrm{C}_{16} \mathrm{H}_{11}=\mathrm{pA}_{4}+\mathrm{H}$ calculated by Sinha et al. [47].

aa Rate constant analogized to $\mathrm{C}_{5} \mathrm{H}_{6} \mathrm{C}_{5} \mathrm{H}_{5}=\mathrm{C}_{5} \mathrm{H}_{5} \mathrm{C}_{5} \mathrm{H}_{5}+\mathrm{H}$ calculated by Wang et al. [49].

bb Rate constant analogized to $\mathrm{C}_{5} \mathrm{H}_{5}+\mathrm{CH}_{3}=\mathrm{C}_{5} \mathrm{H}_{5} \mathrm{CH}_{3}$ calculated by Sharma and Green [51].

cc Rate constant analogized to $\mathrm{A}_{1} \mathrm{CH}_{2}=\mathrm{C}_{5} \mathrm{H}_{5}+\mathrm{C}_{2} \mathrm{H}_{2}$ estimated in the model of Yuan et al. [56].

dd Rate constant analogized to $\mathrm{C}_{5} \mathrm{H}_{7}=\mathrm{C}_{5} \mathrm{H}_{6}+\mathrm{H}$ estimated in the model of Gueniche et al. [89].

ee Rate constant analogized to $\mathrm{C}_{6} \mathrm{H}_{5} \mathrm{C}_{6} \mathrm{H}_{5}+\mathrm{H}=\mathrm{C}_{6} \mathrm{H}_{6} \mathrm{C}_{6} \mathrm{H}_{5}$ estimated in the model of Wang and Frenklach [14].

ff Rate constant analogized to $\mathrm{A}_{1}-+\mathrm{H}=\mathrm{A}_{1}$ estimated in the model of Wang and Frenklach [14].

gg Rate constant analogized to $\mathrm{C}_{5} \mathrm{H}_{5} \mathrm{CH}_{3}=\mathrm{C}_{5} \mathrm{H}_{5} \mathrm{CH}_{2}+\mathrm{H}$ calculated by Sharma and Green [51].

hh Rate constant analogized to $\mathrm{C}_{5} \mathrm{H}_{5} \mathrm{CH}_{2}=\mathrm{A}_{1}+\mathrm{H}$ in the model of Harper et al. [90].

ii Rate constant analogized to $\mathrm{C}_{5} \mathrm{H}_{5} \mathrm{C}_{5} \mathrm{H}_{5}+\mathrm{C}_{5} \mathrm{H}_{5}=\mathrm{C}_{5} \mathrm{H}_{5} \mathrm{C}_{5} \mathrm{H}_{4}+\mathrm{C}_{5} \mathrm{H}_{6}$ calculated by Matsugi and Miyoshi [57].

\subsection{Indene $\left(\mathrm{C}_{9} \mathrm{H}_{8}\right)$ decomposition reactions}

\subsubsection{Indenyl $\left(\mathrm{C}_{9} \mathrm{H}_{7}\right)$ formation}

Indenyl radical is the most critical intermediate in the growth of PAH from indene. Indenyl may

be formed via the combination of smaller aromatic species [10,79]; however, it is largely derived from indene. H-elimination (R1) and H-abstraction (R2) are the dominant reactions in the consumption of indene, both resulting in the formation of indenyl. As shown in Scheme 1, C-H bond energy of the $1^{\circ}$ site is $1.2 \mathrm{kcal} / \mathrm{mol}$ lower than its weakest $\mathrm{C}-\mathrm{C}$ bonds. Unimolecular $\mathrm{C}-\mathrm{H}$ decomposition should be favored compared to the unimolecular C-C bond fission. Laskin and Lifshitz [62] estimated the rate constant for $\mathrm{C}-\mathrm{H}$ bond dissociation of indene $(\mathrm{R} 1)$ to be $1.1 \times 10^{15} \mathrm{e}^{-77000 / R T} \mathrm{~cm}^{3} \cdot \mathrm{mol}^{-1} \cdot \mathrm{s}^{-1}$, which is pressure-independent. However, different values were adopted in later models [7,8,66,77]. For example, Lifshitz et al. [66] and Pousse et al. [77] also considered R1 to be pressure-independent and temperature-independent; they estimated the rate constant to be $6.0 \times 10^{13}$ and $1.0 \times 10^{14} \mathrm{~cm}^{3} \cdot \mathrm{mol}^{-1} \cdot \mathrm{s}^{-1}$, respectively. Blanquart et al. [7] proposed R1 in its reverse direction and recommended its value to be same as that of cyclopentadiene, $1.73 \times 10^{68} \mathrm{~T}^{-15.16} \mathrm{e}^{-116372 / \mathrm{RT}} \mathrm{s}^{-1}$. Laskin and Lifshitz [62] evaluated the rate constant of $\mathrm{R} 2$ as $1.0 \times 10^{14} \mathrm{e}^{-5500 / \mathrm{RT}} \mathrm{cm}^{3} \cdot \mathrm{mol}^{-1} \cdot \mathrm{s}^{-1}$ according to the measurements of 
209 cyclopentadiene decomposition by Roy et al. [91]. In some literature models, this rate constant was

210 also proposed in analogy to $\mathrm{H}$-abstraction reactions of other alkenes [3,92,93]. In this study, we adopt

211 the rate constants of R1 and R2 analogously to those of cyclopentadiene. Here, R1 is taken from the

212 H-elimination of cyclopentadiene in Jet-surf 2.0 model [82], which was also adopted in some other

213 PAH models $[8,56,77]$. The rate constant of R2 is referred to the calculations of Robinson and

214 Lindstedt [34] on cyclopentadiene.

(a)

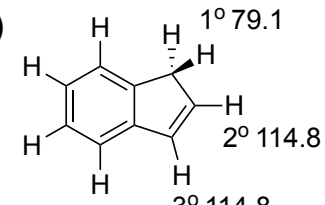

(b)

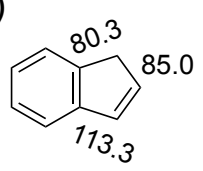

Scheme 1 (a) C-H and (b) C-C bond energies of indene, calculated at $298 \mathrm{~K}$. Unit: $\mathrm{kcal} / \mathrm{mol}$.

\subsubsection{Indenyl $\left(\mathrm{C}_{9} \mathrm{H}_{7}\right)$ and indanyl $\left(\mathrm{C}_{9} \mathrm{H}_{9}\right)$ decomposition reactions}

Indenyl radical is very difficult to decompose at intermediate temperatures $(\sim 1200 \mathrm{~K})$ compared

219 to other fuel radicals $[62,94,95]$. According to the models of Yuan et al. [56], Slavinskaya et al. [39],

220 and Matsugi and Miyoshi [57], three decomposition pathways of indenyl are included in present model,

221 forming cyclopentadienyl + butadiyne, benzyne + propargyl, and fulvenallenyl + acetylene.

H-addition reactions of indene (R3-R4) form two indanyl isomers $\left(\mathrm{C}_{9} \mathrm{H}_{9}{ }^{1}\right.$ : radical on $1^{\circ}$ site, $\mathrm{C}_{9} \mathrm{H}_{9}{ }^{2}$ :

223 radical on $2^{\circ}$ site). The sub-mechanism of indanyl radical was studied by Pousse et al. [77]. Here, we

224 include that part of their model in the present model. Further reactions of two indanyl isomers will be 225 analyzed in the discussion section (Section 5.1.2).

\section{2 РАH formation via indenyl reactions}

PAH formation mechanism is too complicated to be fully reviewed in this section. We will limit

229 to the specific molecule of this study. $\mathrm{C}_{9} \mathrm{H}_{7}$ has three isomers, labelled $\mathrm{C}_{9} \mathrm{H}_{7}{ }^{1}$ (indenyl), $\mathrm{C}_{9} \mathrm{H}_{7}{ }^{2}$ and

$230 \mathrm{C}_{9} \mathrm{H}_{7}{ }^{3}$. However, considering the high energy barrier (> $100 \mathrm{kcal} / \mathrm{mol}$, see $S M M 3$ ) of H-shift from

231 indenyl to other isomers $\left(\mathrm{C}_{9} \mathrm{H}_{7}{ }^{2}\right.$ and $\left.\mathrm{C}_{9} \mathrm{H}_{7}{ }^{3}\right)$, indenyl is the most favorable precursor of further PAH 
232 growth pathways. Hence, the formation pathways of $\mathrm{C}_{10}-\mathrm{C}_{18}$ PAHs in this work are considered based

233 on the indenyl radical.

\subsection{1. $C_{10}$ aromatic species}

The mechanism of $\mathrm{C}_{10}$ species has been studied in detail in literature $[66,78,96]$. It mainly includes the reactions of methyl-indene isomers, $\mathrm{C}_{10} \mathrm{H}_{9}$ radicals, naphthalene $\left(\mathrm{C}_{10} \mathrm{H}_{8}\right)$, describing the conversion from indenyl to naphthalene via the addition of a methyl radical. Reactions of methyl-indene were considered to be rather important pathway in naphthalene formation [53]; therefore, we included all three methyl-indene $\left(\mathrm{C}_{10} \mathrm{H}_{10}\right)$ isomers in the present model. As calculated by Dubnikova and Lifshitz [78], the isomerization reactions of $\mathrm{C}_{10} \mathrm{H}_{10}$ and $\mathrm{C}_{10} \mathrm{H}_{9}$ species are very quick. 1,5 H-shift could also easily occur in cyclopentadienyl structure [46,51], thus 3-methyl-indene could be a more favorable intermediate due to its lower energy among the three isomers [78]. As shown in Table 1, combination of indenyl and methyl radicals forms 1-mehtyl-indene (R5). It later isomerizes to 2- and 3-methylindene. Further decomposition and $\mathrm{H}$-abstraction reactions of methyl-indene isomers produce various $\mathrm{C}_{10} \mathrm{H}_{9}$ radicals. Well-skipping reaction between indenyl and methyl may directly form $\mathrm{C}_{9} \mathrm{H}_{6}{ }^{3} \mathrm{CH}_{3}$ radical (R16). According to the calculations of Dubnikova and Lifshitz [78], only three $\mathrm{C}_{10} \mathrm{H}_{9}$ radicals, 3-methyl-1-indenyl $\left(\mathrm{C}_{9} \mathrm{H}_{6}{ }^{3} \mathrm{CH}_{3}\right)$, 1-indenyl-methyl $\left(\mathrm{C}_{9} \mathrm{H}_{7}{ }^{1} \mathrm{CH}_{2}\right)$ and 2-indenyl-methyl $\left(\mathrm{C}_{9} \mathrm{H}_{7}{ }^{2} \mathrm{CH}_{2}\right)$, are considered to be favorable in the subsequent isomerization reactions. Further $\mathrm{C}_{10} \mathrm{H}_{9}$ isomerization reactions (R6-R13) yield $\alpha$ - and $\beta$-naphthyl radicals $\left(\mathrm{aC}_{10} \mathrm{H}_{9}\right.$ and $\left.\mathrm{bC}_{10} \mathrm{H}_{9}\right)$, which are naphthalene $\left(\mathrm{C}_{10} \mathrm{H}_{8}\right)$ precursors (R14 and $\left.\mathrm{R} 15\right)$. In addition, ipso-reactions of methyl-indene isomers yield methyl and indene (R17-R19).

\subsection{2. $C_{11}$ aromatic species}

We considered two groups of species for $\mathrm{C}_{11}$ aromatics: ethynyl-indene and methyl-naphthalene. The combination between indenyl and acetylene is proposed in this model to form naphthyl-methyl $\left(\mathrm{A}_{2}{ }^{1} \mathrm{CH}_{2}\right)$ and benzo-cycloheptatrien-5-yl $\left(\mathrm{cC}_{11} \mathrm{H}_{9}\right)$ radicals. Ethynyl-indene and methyl-naphthalene are then formed in a few elementary steps, as shown in Table 1. R20-R26 are analogized to those of 
257 cyclopentadienyl [64] in the present model; their rate constants are taken from the calculations in

258 literature $[64,79,83,84]$.

\subsection{3. $C_{12}$ aromatic species}

Two $\mathrm{C}_{12} \mathrm{H}_{8}$ isomers are considered in the present model, acenaphthylene $\left(\mathrm{A}_{2} \mathrm{R}_{5}\right)$ and ethynylnaphthalene $\left(\mathrm{A}_{2} \mathrm{C}_{2} \mathrm{H}\right)$. Acenaphthylene, having fused six- and five-member rings, is an important intermediate in PAH growth. According to the literature models, it is either proposed to be formed

263 through the combination of indenyl and propargyl [7,10,97] or the cyclization of naphthyl-2-vinyl 264 radical $\left(\mathrm{A}_{2} \mathrm{C}_{2} \mathrm{H}_{2}\right)[14,16]$. A global rate constant used in literature models $[8,56]$ is referred to the 265 estimation of Blanquart et al. [7], which was based on the assumption that indenyl radical gets a benzyl266 like structure by its reaction with propargyl (see the part of molecule highlighted in red in Scheme 2). 267 The benzyl-like part in indenyl is added by propargyl, and then cyclizes to form another benzene ring 268 inbetween the six- and five-member rings of indene. As a result, this reaction consumes two relatively 269 less reactive radicals $\left(\mathrm{C}_{9} \mathrm{H}_{7}\right.$ and $\left.\mathrm{C}_{3} \mathrm{H}_{3}\right)$, but produces two $\mathrm{H}$-atoms that are much more active. Recent 270 calculations of Long et al. [48] for the combination of two cyclopentadienyl radicals and Sinha et al. 271 [47] for the combination of indenyl and benzyl showed that a necessary H-loss step (via H-abstraction 272 or H-elimination reactions) from $\left|\mathrm{R}^{\prime}-\mathrm{R}\right|$ to $\left|\mathrm{R}^{\prime}-\mathrm{R}\right|^{*}$ radical ( $\mathrm{R}$ and $\mathrm{R}^{\prime}$ are functional groups, * is a radical 273 label) should be considered in the model, particularly at relatively low temperatures. This approach 274 will effectively adjust the over-estimation of the system reactivity by the global reaction, since it 275 consumes an extra radical in $\mathrm{H}$-abstraction reaction of $\left|\mathrm{R}^{\prime}-\mathrm{R}\right|$ (three radicals in and one out). Therefore, 276 in this work, we proposed a multi-step scheme for the addition of propargyl to indenyl (R27-34) and 277 analogized to that of benzyl $[9,45,56,57]$ but also included a necessary H-abstraction reaction step 278 (R30). Detailed reaction process is shown in Scheme 2. 


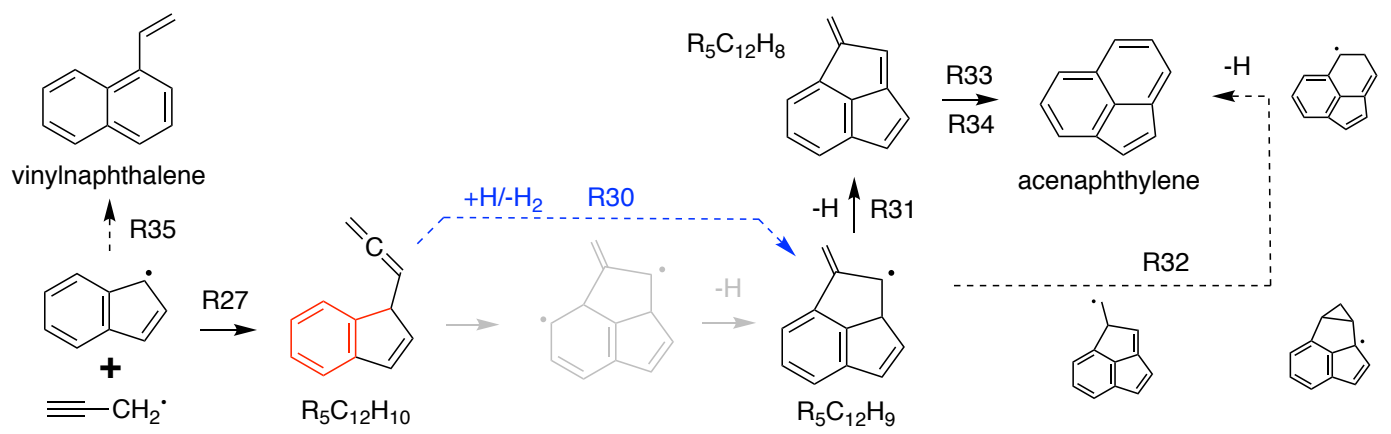

280 Scheme 2 Reaction scheme proposed for $\mathrm{C}_{9} \mathrm{H}_{7}+\mathrm{C}_{3} \mathrm{H}_{3}$. Solid arrows represent elementary reaction steps, dashed arrows represent multiple reaction steps. Blue dashed arrow highlights the key $\mathrm{H}$-abstraction reaction step.

The addition of acetylene on naphthyl radical can produce naphthyl-2-vinyl radical. It either cyclizes to acenaphthylene or forms ethynyl-naphthalene via $\mathrm{C}-\mathrm{H}$ bond cleavage. Herein, we propose 286 (R35), competing with the route proposed for acenaphthylene. This is analogized to the styrene 287 formation reaction by cyclopentadienyl and propargyl [10,40,98].

\subsection{4. $C_{13}$ aromatic species}

Benz[g]indene, benz[f]indene and fluorene are typical $\mathrm{C}_{13} \mathrm{H}_{10}$ PAHs that can be formed via the 290 combination of indenyl and cyclopentadienyl radicals [50]. H-elimination reactions of these molecules 291 yield benz[g]indenyl, benz[f]indenyl and fluorenyl radicals, which are also cyclopentadienyl-like 292 radicals but have tricyclic rings. We proposed two molecular structure groups for $\mathrm{C}_{13} \mathrm{H}_{10}$ and $\mathrm{C}_{13} \mathrm{H}_{9}$ 293 species in the present model: one group represents the benz-indene isomers $\left(\mathrm{bC}_{13} \mathrm{H}_{10}\right)$ and their radicals $294\left(\mathrm{bC}_{13} \mathrm{H}_{9}\right)$ while the other group represents fluorene $\left(\mathrm{fC}_{13} \mathrm{H}_{10}\right)$ and fluorenyl radical $\left(\mathrm{fC}_{13} \mathrm{H}_{9}\right)$. Benz295 indenyl is treated separately from fluorenyl because the chemical reactivity of the five-member ring in 296 this molecule is impacted by one benzene ring on its single side whilst that of fluorenyl is impacted on 297 its both sides. Fluorenyl would be more difficult to undergo internal ring rearrangement than benz298 indenyl radical after its addition reactions since the Pi-bond of fluorenyl is stronger than the double 299 bond of benz-indenyl. Therefore, we consider benz-indenyl to be more active in PAH growth. In 300 addition, fluorenyl and benz-indenyl radicals produce very different large PAHs through subsequent 
reactions with same intermediates. However, benz[g]indenyl and benz[f]indenyl form similar products in further reactions, are thus lumped.

The combination of indenyl and cyclopentadienyl [50] could be similar to that of two 304 cyclopentadienyls [43,48]. However, the reactions of $\mathrm{C}_{9} \mathrm{H}_{7}+\mathrm{C}_{5} \mathrm{H}_{6}$ and $\mathrm{C}_{9} \mathrm{H}_{8}+\mathrm{C}_{5} \mathrm{H}_{5}$ are slightly 305 different from $\mathrm{C}_{5} \mathrm{H}_{5}+\mathrm{C}_{5} \mathrm{H}_{6}$ because of the non-symmetrical structures of $\mathrm{C}_{9} \mathrm{H}_{7} \mathrm{C}_{5} \mathrm{H}_{6}$ and $\mathrm{C}_{9} \mathrm{H}_{8} \mathrm{C}_{5} \mathrm{H}_{5}$ 306 (see their structures in Scheme 3). These will either eliminate an H-atom forming $\mathrm{C}_{9} \mathrm{H}_{7} \mathrm{C}_{5} \mathrm{H}_{5}$ or 307 undergo isomerization steps forming $\mathrm{fC}_{13} \mathrm{H}_{10} \mathrm{CH}_{3}$ and $\mathrm{bC}_{13} \mathrm{H}_{10} \mathrm{CH}_{3}$ radicals. The products of these two 308 channels are similar, mainly fluorene and benzo-indene. We propose the following scheme (R36-43) 309 based on the calculations of Mulholland et al. [50]; rate constants are analogized to the similar reactions 310 between cyclopentadienyl and cyclopentadiene [86].

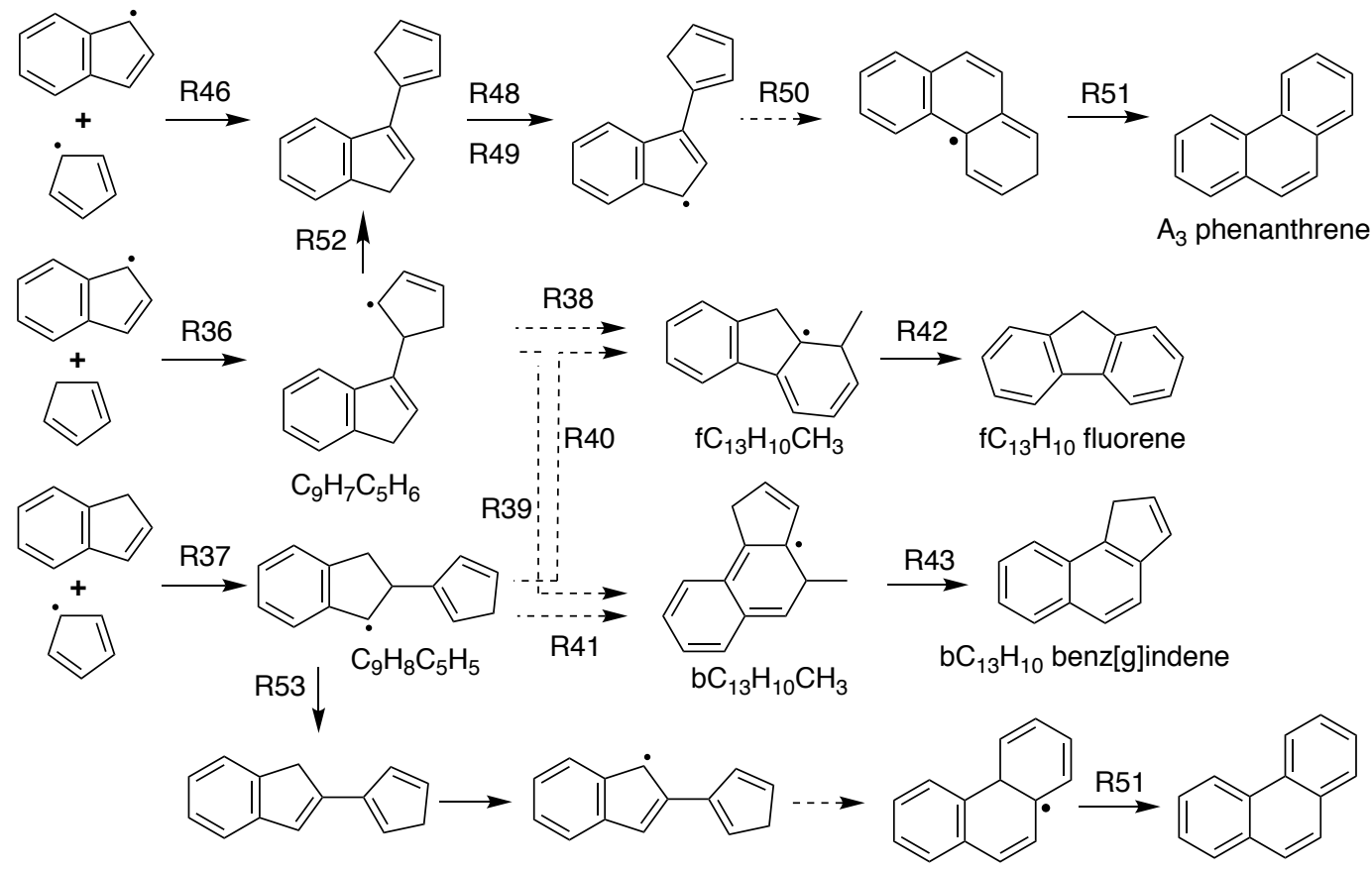

312 Scheme 3 Reaction scheme proposed for $\mathrm{C}_{9} \mathrm{H}_{8}+\mathrm{C}_{5} \mathrm{H}_{5}$ and $\mathrm{C}_{9} \mathrm{H}_{7}+\mathrm{C}_{5} \mathrm{H}_{6}$. Solid arrows represent elementary reaction steps; dashed arrows represent reactions with multiple steps.

In addition to the reactions suggested by Mulholland et al. [50], we also propose a formation 315 pathway of benz-indene from naphthylmethyl (R44), similar to the formation of indene from the combination of benzyl and acetylene [88]. For the combination of propargyl and naphthalene (R45)

$317[19,56]$, we recommend its product to be benz-indene instead of fluorene because the addition of 
318 propargyl is more likely to create a fused five-member ring on one side than in the middle of

319 naphthalene.

\subsection{5. $C_{14}$ aromatic species}

$\mathrm{C}_{14} \mathrm{H}_{10}$ and $\mathrm{C}_{14} \mathrm{H}_{12}$ are the main groups of $\mathrm{C}_{14} \mathrm{PAHs}$ considered in the kinetic models. Anthracene and phenanthrene are the $\mathrm{C}_{14} \mathrm{H}_{10}$ isomers identified in previous studies, and are typical tricyclic aromatic species. In our model, we lumped these two isomers, since they have very similar growth pathways and yield products with similar structures. Beside HACA formation pathways for $\mathrm{C}_{14} \mathrm{H}_{10}$ PAHs [3,14], we propose the combination of indenyl and cyclopentadienyl (R46-51) to be an efficient route [50] (see Scheme 3). The rate constants of R46-51 are again analogized to that of 327 cyclopentadienyl (see Table 1).

For $\mathrm{C}_{14} \mathrm{H}_{12}$ species, Yuan et al. [56] and Matsugi and Miyoushi [57] proposed stilbene to be a 329 possible structure in their modeling studies of toluene combustion. However, benzyl radical 330 concentration is much less than indenyl in the present study; stilbene is thus not that important 331 compared to the products derived from indenyl reaction sequences. Therefore, we assumed $\mathrm{C}_{14} \mathrm{H}_{12}$ to 332 be methyl-benzindene or methyl-fluorene formed through the combination of methyl and benz-indenyl 333 or fluorenyl (R54-55). Further reactions of both species can form phenanthrene, similar to the 334 transformation from methyl-cyclopentadiene to benzene [51].

\subsection{6. $C_{15}$ aromatic species}

Methyl-phenanthrene $\left(\mathrm{A}_{3} \mathrm{CH}_{3}\right)$ can be formed via many pathways; simplest one is the methyl 337 addition to phenanthrene. When methyl-phenanthrene loses an H-atom, phenanthrenyl-methyl radical $338\left(\mathrm{~A}_{3} \mathrm{CH}_{2}\right)$ is formed. This radical can also be produced by the combination of benz-indenyl and 339 acetylene (R52), which is similar to the conversion from cyclopentadienyl to benzyl. Cyclization of 340 phenanthrenyl-methyl radical yields another radical (R57), see $\mathrm{cC}_{15} \mathrm{H}_{11}$ in Scheme 4. Thereafter, $4 \mathbf{H}$ 341 cyclopenta[def]phenanthrene $\left(\mathrm{cpC}_{15} \mathrm{H}_{10}\right)$ can be formed via $\beta$-scission of its $\mathrm{C}-\mathrm{H}$ bond (R62). We also 342 proposed $\mathrm{cpC}_{15} \mathrm{H}_{10}$ to be formed through the combination of indenyl and phenyl. As shown in Scheme 
$344 \mathrm{cC}_{15} \mathrm{H}_{11}$ radical via seven-member-ring structure intermediates. A brief reaction scheme (R58-61) is 345 shown in Table 1 with estimated rate constants. Opening of double bonds in $\mathrm{C}_{9} \mathrm{H}_{7} \mathrm{C}_{6} \mathrm{H}_{5}$ and further 346 cyclization is considered negligible, as shown by the red cross in Scheme 4.

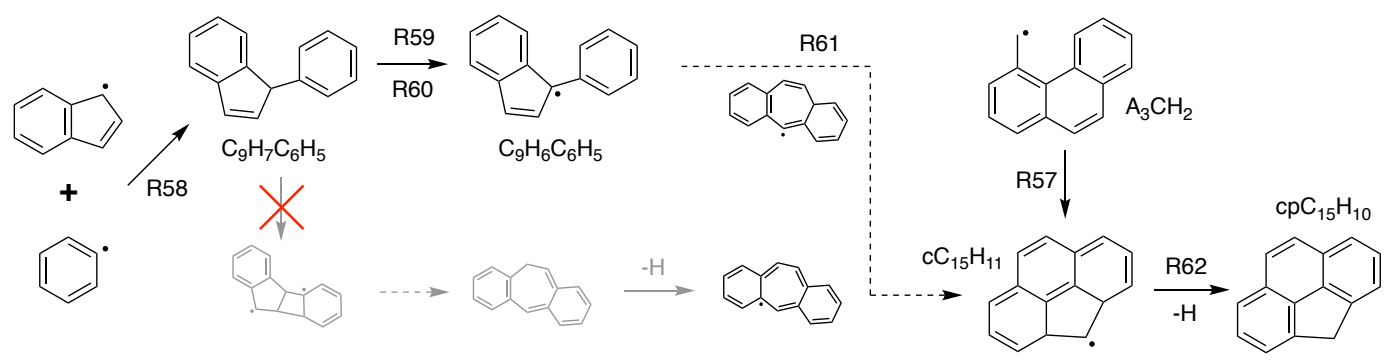

348 Scheme 4 Reaction scheme proposed for $\mathrm{C}_{9} \mathrm{H}_{7}+\mathrm{C}_{6} \mathrm{H}_{5}$. Solid arrows represent elementary reaction steps; dashed arrows represent reactions with multiple steps.

\subsection{7. $C_{16}$ aromatic species}

$\mathrm{C}_{16} \mathrm{H}_{10}$ has at least four tetracyclic isomers that can be detected and should be considered in the

352 PAH model; these are pyrene, fluoranthene, acephenanthrylene and aceanthrylene. According to the 353 previous studies, pyrene is proposed to be the dominant isomer among these $[3-5,7,9,10,57]$. However, 354 recent atomic force microscopy measurements on PAHs has shown a significance of the five-member355 ring in their molecular structures [99]. Therefore, acephenanthrylene and aceanthrylene could still be 356 important in $\mathrm{PAH}$ formation, although they cannot be easily distinguished from other $\mathrm{C}_{16} \mathrm{H}_{10}$ isomers. 357 The sub-mechanisms of acephenanthrylene and aceanthrylene are not included in the present model 358 due to the lack of their kinetic data. These sub-mechanisms are not closely related to indenyl reactions 359 and could be completed in a future study. We added the reaction of indenyl and benzyl in the present 360 model (R63-R73 in Table 1) for pyrene $\left(\mathrm{pA}_{4}\right)$ and fluoranthene $\left(\mathrm{fC}_{16} \mathrm{H}_{10}\right)$, which are adopted from the 361 quantum chemical calculations of Sinha et al. [47]. After the combination of indenyl and benzyl (R63), 362 the product $\mathrm{C}_{9} \mathrm{H}_{7} \mathrm{C}_{7} \mathrm{H}_{7}$ undergoes several reaction steps (R64-R67) to form phenyl-naphthalene $363\left(\mathrm{C}_{10} \mathrm{H}_{7} \mathrm{C}_{6} \mathrm{H}_{5}\right)$. The following reaction sequence of $\mathrm{C}_{10} \mathrm{H}_{7} \mathrm{C}_{6} \mathrm{H}_{5}(\mathrm{R} 68-\mathrm{R} 73)$ is the same as the reaction 364 pathway of naphthyl and phenyl combination. The addition reaction of methyl to $\mathrm{cpC}_{15} \mathrm{H}_{9}$ is also 365 considered a potential pyrene formation pathway (R74-R78). Besides these routes, we propose the 
366 addition of propargyl on fluorenyl to yield fluoranthene, analogized to the addition of propargyl on 367 benz-indenyl [97].

\subsection{8. $C_{17}$ aromatic species}

$\mathrm{C}_{17} \mathrm{PAH}$ species have not been detailed in previous studies [11,100,101]; particularly their specific formation pathways and carbon growth reactions are not described adequately. Previous quantum chemical calculations pointed out that the combination of indenyl and indene can form $\mathrm{C}_{17}$ and $\mathrm{C}_{18}$ species [65]. Three benzofluorene isomers may be formed in this reaction scheme, benzo[b]fluorene (BCF) is emphasized as a major isomer [65]. As shown in Scheme 5a, Kislov et al.

374 [86] calculated a series of $\mathrm{C}_{10} \mathrm{H}_{11}$ isomerization reactions from 3a,4,7,7a-tetrahydro- $1 H$-4,7-methano375 indenyl (INT1) to 7-methyl-7,7a-dihydro-1H-7a-indenyl (INT3) after the combination of cyclopentadienyl and cyclopentadiene. $\beta$-Scission of INT3 preferentially produces indene rather than methyl-indene because of the weaker dissociation energy of $\mathrm{C}-\mathrm{C}$ bond than $\mathrm{C}-\mathrm{H}$ bond. A modified 378 scheme for $\mathrm{C}_{9} \mathrm{H}_{8}+\mathrm{C}_{9} \mathrm{H}_{7}\left(\mathrm{R} 79-\mathrm{R} 81\right.$ in Table 1) is adopted in the present model analogously to $\mathrm{C}_{5} \mathrm{H}_{6}+$ $\mathrm{C}_{5} \mathrm{H}_{5}$ reactions, as shown in Scheme $\mathbf{5 b}$.

(a)

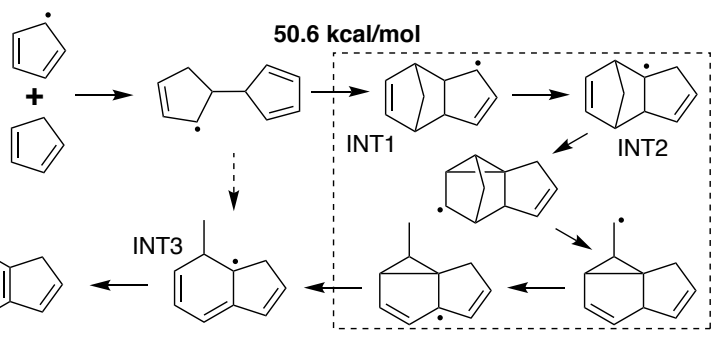

(b)<smiles>c1ccc2ccccc2c1</smiles><smiles>C1=Cc2ccccc2C1</smiles><smiles>c1ccc2c(c1)Cc1cc3ccccc3cc1-2</smiles>

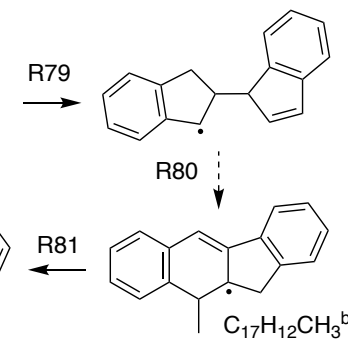

Scheme 5 Reaction schemes (a) for $\mathrm{C}_{5} \mathrm{H}_{6}+\mathrm{C}_{5} \mathrm{H}_{5}$ calculated by Kislov et al. [86], (b) proposed here for $\mathrm{C}_{9} \mathrm{H}_{8}+\mathrm{C}_{9} \mathrm{H}_{7}$. Solid arrows represent elementary reaction steps; broad arrows represent reactions with multiple steps. 


\subsection{9. $C_{18}$ aromatic species}

390 We consider three kinds of $\mathrm{C}_{18}$ PAH species in the present model, with formulae of $\mathrm{C}_{18} \mathrm{H}_{14}, \mathrm{C}_{18} \mathrm{H}_{12}$ 391 and $\mathrm{C}_{18} \mathrm{H}_{10} . \mathrm{C}_{18} \mathrm{H}_{14}$ could be 5-methyl-benzofluorene $\left(\mathrm{BCF}^{5} \mathrm{CH}_{3}\right)$, formed by the $\beta$-scission of $392 \mathrm{C}_{17} \mathrm{H}_{12} \mathrm{CH}_{3}{ }^{\mathrm{b}}$ (R86) [65]. Another methyl-benzofluorene isomer, labeled as 11-methyl-benzofluorene $393\left(\mathrm{BCF}^{11} \mathrm{CH}_{3}\right)$, is is the product of the addition of methyl and benzo-fluorenyl radicals (R87). Bi-indene 394 isomers are dominant $\mathrm{C}_{18} \mathrm{H}_{14}$ species $[63,65]$. In the previous studies of cyclopentadienyl combination $395[46,48]$, the intermediate $1,1-\mathrm{C}_{5} \mathrm{H}_{5} \mathrm{C}_{5} \mathrm{H}_{5}$ ([1,1'-bi(cyclopentane) $]-2,2$ ',4,4'-tetraene) can easily 396 isomerize to other structures, such as 2,2- $\mathrm{C}_{5} \mathrm{H}_{5} \mathrm{C}_{5} \mathrm{H}_{5}, 2,3-\mathrm{C}_{5} \mathrm{H}_{5} \mathrm{C}_{5} \mathrm{H}_{5}$ and 3,3- $\mathrm{C}_{5} \mathrm{H}_{5} \mathrm{C}_{5} \mathrm{H}_{5}$. Bi-indene has 397 similar isomers and reaction network. We lumped all bi-indene isomers as 3,3-bi-indene in order to 398 simplify the present model because all isomers have very similar further reactions [48]. This is the 399 same strategy adopted in the model of Yuan et al. for cyclopentadienyl combination reactions [56]. 400 The related reactions of indenyl combination (R88 and R89 in Table 1) are analogized to those of 401 cyclopentadienyl, as shown in Scheme 6. Furthermore, the combinations of $\mathrm{C}_{13} \mathrm{H}_{9}$ isomers and 402 cyclopentadienyl produce other $\mathrm{C}_{18} \mathrm{H}_{14}$ isomers ( $\mathrm{R} 90$ and R91), fluorenyl-cyclopentadiene $403\left(\mathrm{fC}_{13} \mathrm{H}_{9} \mathrm{C}_{5} \mathrm{H}_{5}\right)$ and benzindenyl-cyclopentadiene $\left(\mathrm{bC}_{13} \mathrm{H}_{9} \mathrm{C}_{5} \mathrm{H}_{5}\right)$.

404 Three $\mathrm{C}_{18} \mathrm{H}_{12}$ isomers, chrysene $\left(\mathrm{C}_{18} \mathrm{H}_{12}{ }^{\mathrm{c}}\right)$, benzo[c]phenanthrene $\left(\mathrm{C}_{18} \mathrm{H}_{12}^{\mathrm{p}}\right)$, and triphenylene $405\left(\mathrm{C}_{18} \mathrm{H}_{12}{ }^{\mathrm{t}}\right)$, are considered in the present model. Among these, chrysene is a typical $\mathrm{C}_{18} \mathrm{H}_{12} \mathrm{PAH}$ that 406 has been frequently studied in previous models $[3,7,14,15,18,19,39,55,56] . \mathrm{C}_{9} \mathrm{H}_{7} \mathrm{C}_{9} \mathrm{H}_{7}$ eliminates an 407 H-atom yielding $\mathrm{C}_{9} \mathrm{H}_{6} \mathrm{C}_{9} \mathrm{H}_{7}$ radical, and it isomerizes to hydrochrysenyl $\left(\mathrm{C}_{18} \mathrm{H}_{13}{ }^{\mathrm{c}}\right.$, $\left.\mathrm{R} 95\right)$ or 408 hydrobenzo[c]phenanthrenyl $\left(\mathrm{C}_{18} \mathrm{H}_{13}{ }^{\mathrm{p}}\right.$, R96). The $\beta$-scission reactions of $\mathrm{C}_{18} \mathrm{H}_{13}{ }^{\mathrm{c}}$ and $\mathrm{C}_{18} \mathrm{H}_{13}{ }^{\mathrm{p}}$ form 409 chrysene and benzo[c]phenanthrene (R97 and R98), respectively. Another formation channel of $410 \mathrm{C}_{9} \mathrm{H}_{6} \mathrm{C}_{9} \mathrm{H}_{7}$ radical is the well-skipping reaction of two indenyl radicals (R99). The proposed formation 411 pathway for chrysene is shown in $\boldsymbol{S c h e m e} \mathbf{6} \boldsymbol{b}$, while similar reactions for benzo[c]phenanthrene are 412 omitted. 
(a)

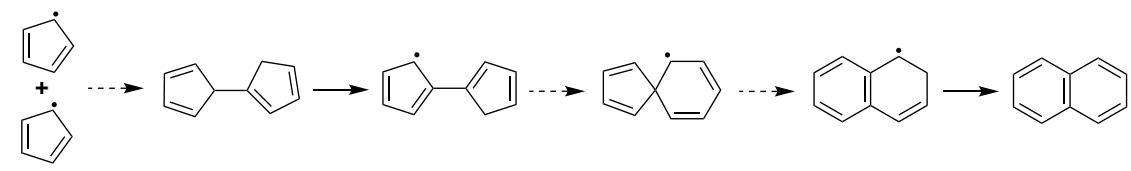

(b)
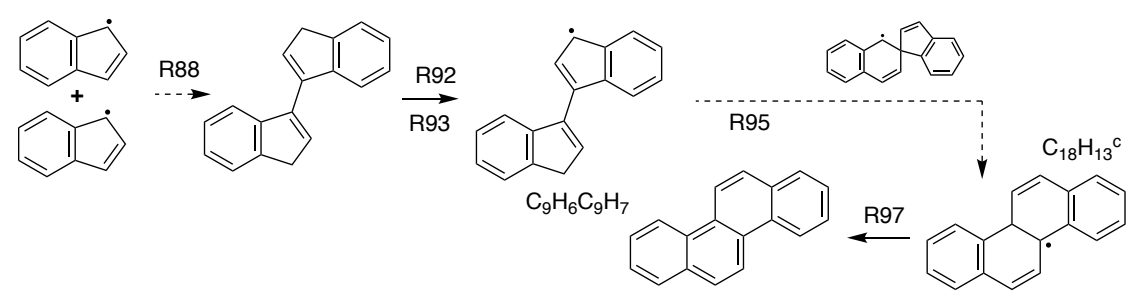

414 Scheme 6 Reaction schemes (a) for $\mathrm{C}_{5} \mathrm{H}_{5}+\mathrm{C}_{5} \mathrm{H}_{5}$, (b) proposed for $\mathrm{C}_{9} \mathrm{H}_{7}+\mathrm{C}_{9} \mathrm{H}_{7}$. Solid arrows represent elementary reaction steps; dash arrows represent reactions with multiple steps. Subsequent reactions of benzindenyl-cyclopentadiene $\left(\mathrm{bC}_{13} \mathrm{H}_{9} \mathrm{C}_{5} \mathrm{H}_{5}\right)$ may also form chrysene and

417 benzo[c]phenanthrene (R100-R104). Slightly different reactions of fluorenyl and cyclopentadienyl 418 yields triphenylene (R105-R109).

\subsubsection{PAHs beyond 18 C-atoms}

$420 \quad$ Since these species are not directly observed in the current experiments, we have provided detailed 421 information of this part in Section 3 of SMM2. Simple reaction schemes are included for some $\mathrm{C}_{19}$ and $422 \mathrm{C}_{20}$ species, as collected in Table S2. Self-combination of large resonantly stabilized radicals were 423 considered for larger PAHs in previous studies [59,61]. In the present model, global reactions are 424 applied for these pathways.

\subsection{Thermodynamic and transport data}

Thermodynamic and transport data of $\mathrm{C}_{0}-\mathrm{C}_{6}$ species used in this work are taken from literature 428 models [56,70], and those of aromatic species are taken from references $[7,14,57,97,102]$. Thermo 429 data of new species added in the present model are calculated by group-additivity method [103-105].

\section{Model validation}

In this section, the present model is validated against the current flow reactor experimental data and the shock tube data of Laskin and Lifshitz [62]. Numerical simulations of flow reactor and shock 
434 tube was performed with OpenSMOKE++ [106]. Energy equation was not solved in the flow reactor;

435 instead temperature profiles measured in the experiment were used as input parameters (see $S M M 1$ ).

436 Shock tube was modelled as an adiabatic batch reactor, and the residence time was set to $1.7 \mathrm{~ms}$

437 according to the experimental method reported in [107]. The main dissociation channels of indene and 438 ring expansion routes from indenyl radical are illustrated based on the kinetic analysis of the present 439 model.

\subsection{Indene pyrolysis in flow reactor}

Dozens of pyrolysis products were detected in the flow reactor pyrolysis experiments of indene,

442 and their mole fraction profiles are plotted as a function of reaction temperature. The experimental and 443 simulated mole fraction profiles of pyrolysis species are shown in Figs. 1-4. In general, the current 444 model can adequately capture the decomposition or formation temperature windows of the observed 445 pyrolysis species, and the quantitative agreement between the experiments and model is quite 446 reasonable. Rate of production (ROP) analysis and sensitivity analysis are used to provide insights into 447 the chemistry of indene decomposition and aromatics growth.
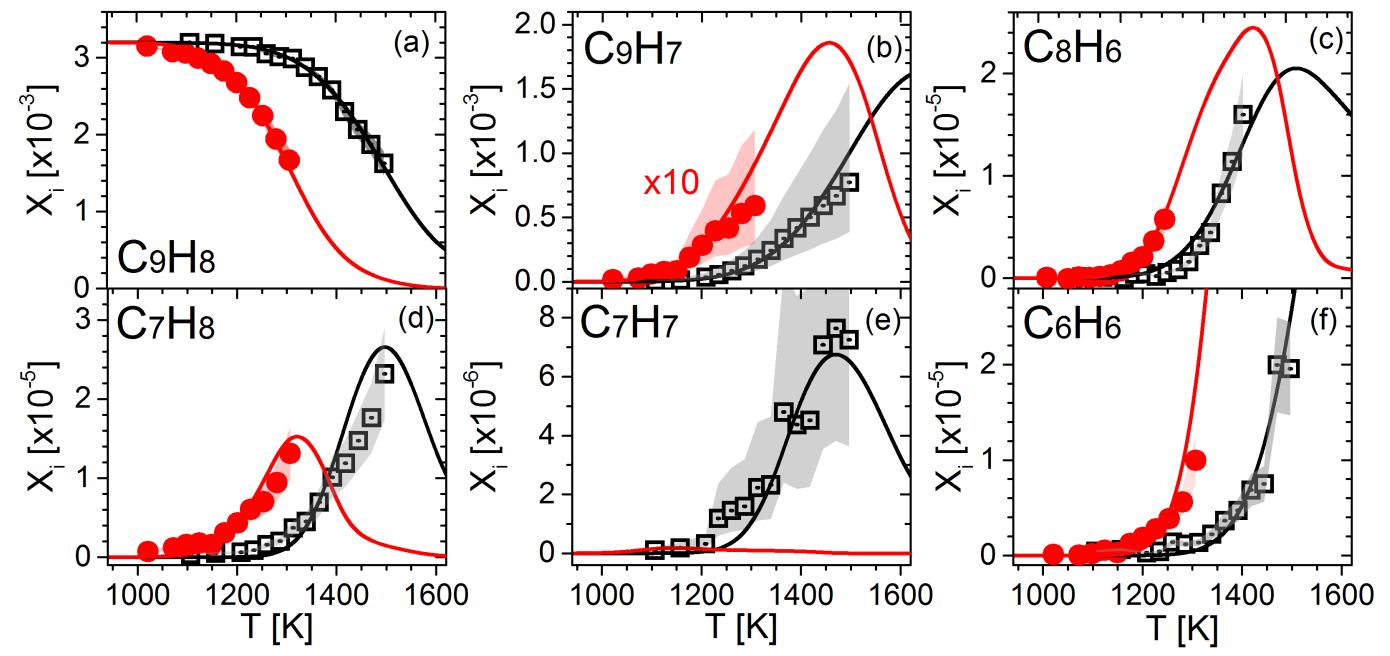

Figure 1 Measured mole fraction profiles of indene $\left(\mathrm{C}_{9} \mathrm{H}_{8}\right)$ and its main decomposition products in a flow reactor at 30 (black squares) and 760 (red circles) Torr. Shadows with the data plots represent the uncertainty of the measurements. Lines are predictions of the present model. 

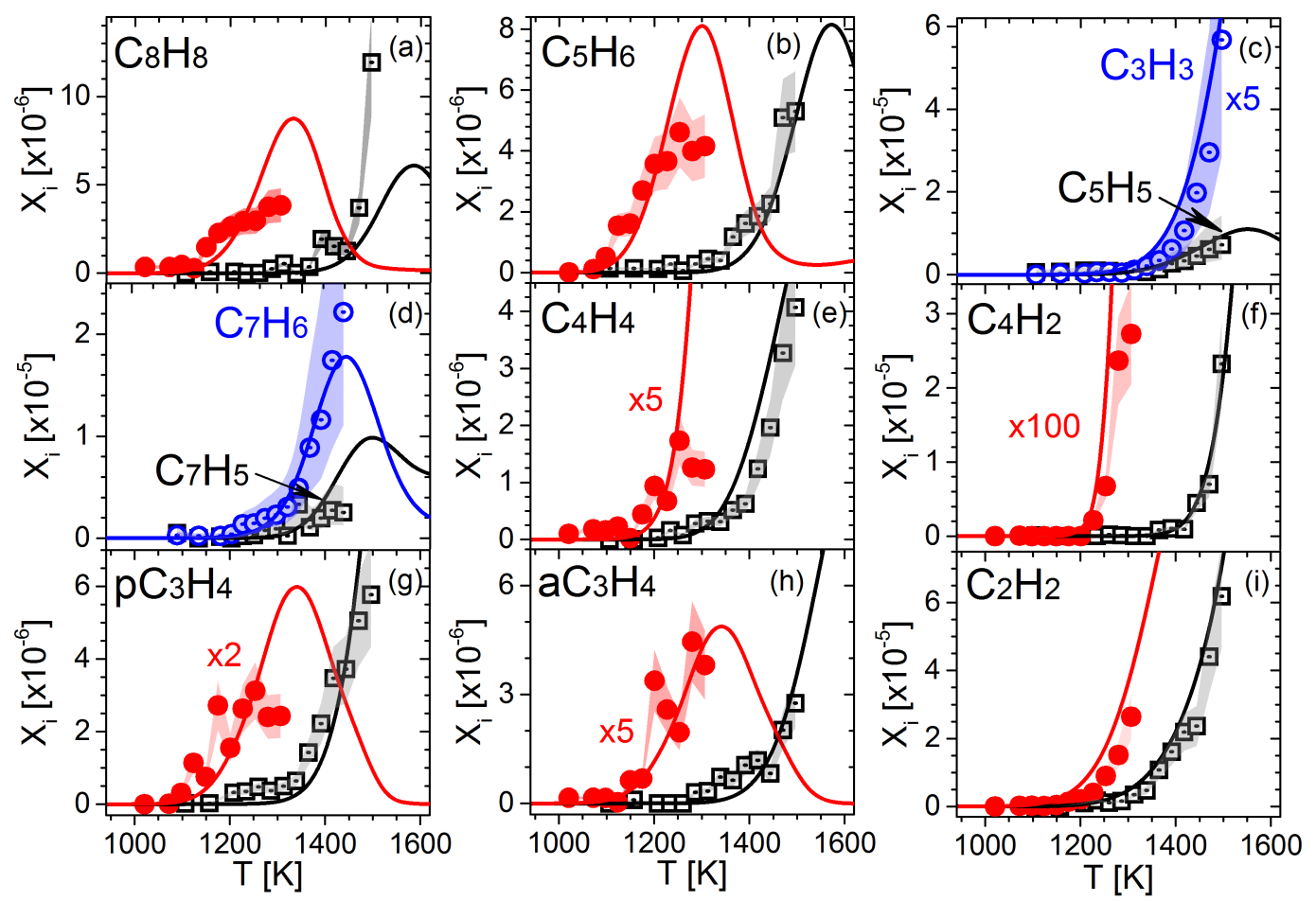

Figure 2 Mole fraction profiles of small intermediates measured in indene pyrolysis in a flow reactor at 30 (black squares) and 760 (red circles) Torr. The blue circles in (c) and (d) are the mole fractions of propargyl and 5-vinylidene-1,3-cyclopentadiene at 30 Torr. Shadows with the data plots represent the uncertainty of the measurements. Lines are the predictions of the present model. Experimental data and simulations of species with small concentrations are scaled with a common factor.
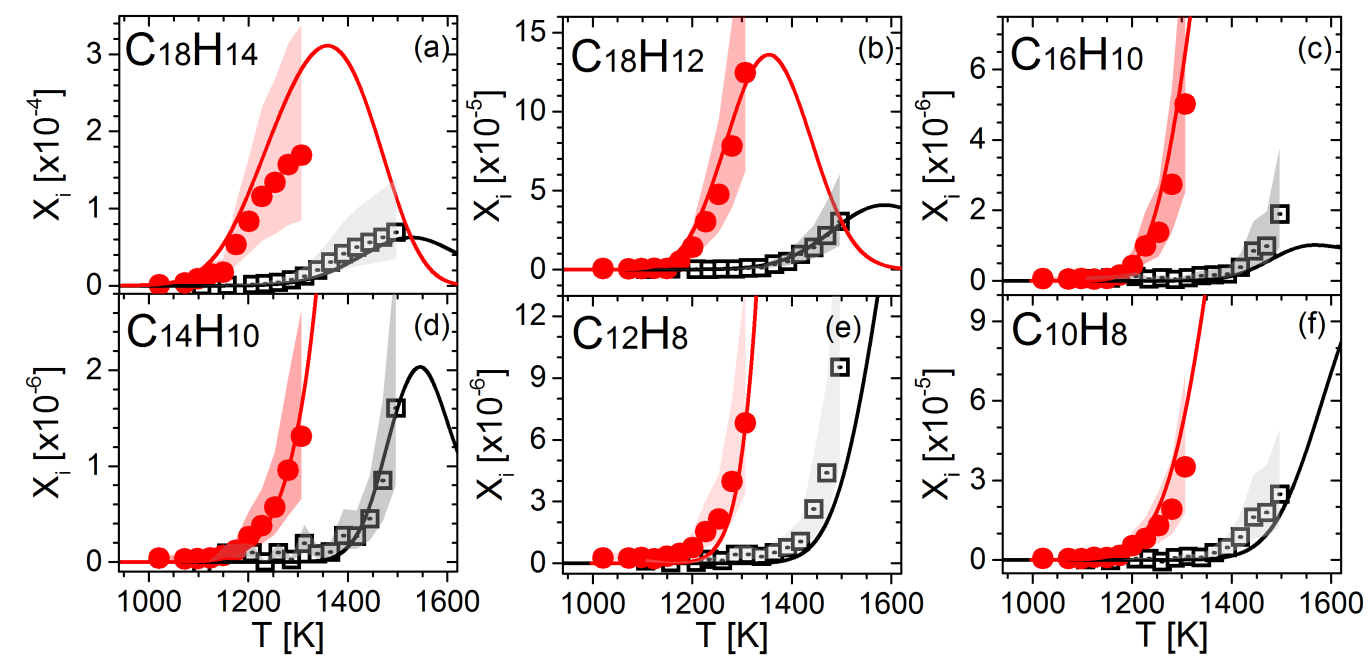

Figure 3 Mole fraction profiles of dominant (common) PAHs measured in indene pyrolysis in a flow reactor at 30 (black squares) and 760 (red circles) Torr. Shadows with the data plots represent the uncertainty of the measurements. Lines indicate the model predictions. 

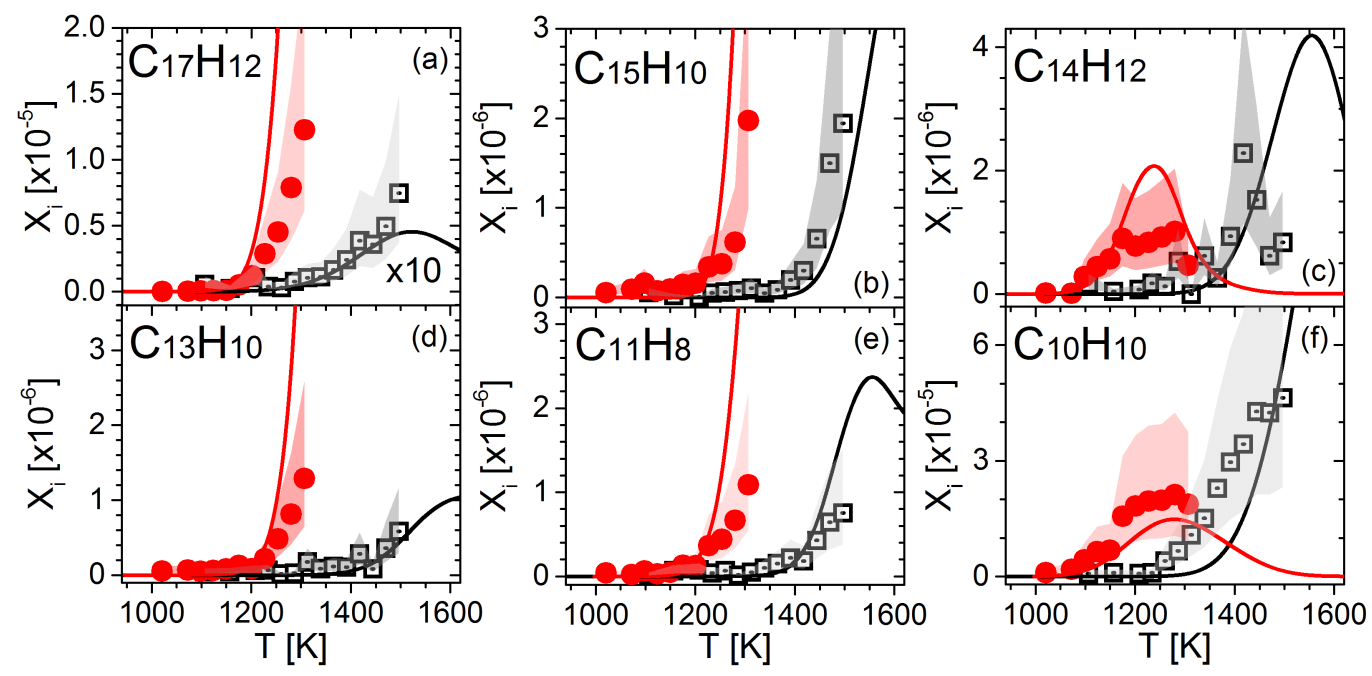

464 Figure 4 Mole fraction profiles of uncommon PAHs measured in indene pyrolysis in a flow reactor at 30 (black squares) and 760 (red circles) Torr. Shadows with the data plots represent the uncertainty of the measurements. Lines indicate the model predictions.

\subsubsection{Primary decomposition of indene}

Indene consumption profiles are presented in Fig. 1(a) and compared with simulation results. Only

471 Torr respectively. Higher conversions of indene at higher temperatures were not measured due to the

472 increasing influence of soot on the sampling process. The present model captured the temperature

473 dependencies of indene consumption at both pressures. ROP analyses were performed under two

474 different conditions at an indene conversion of $17.5 \%$. The reaction networks in the decomposition of

475 indene at both pressures are plotted in Fig. 5. The dominant product of indene decomposition is indenyl

476 radical which accounts for more than $70 \%$ of indene consumption. The low $\mathrm{C}-\mathrm{H}$ bond energy favors a

477 competitive selectivity of $\mathrm{H}$-elimination reaction compared to $\mathrm{C}-\mathrm{C}$ bond dissociation reaction in

478 unimolecular consumption channels. At higher pyrolysis pressure, molecules collide more frequently,

479 so bimolecular H-abstraction reaction takes dominance in indene consumption. 


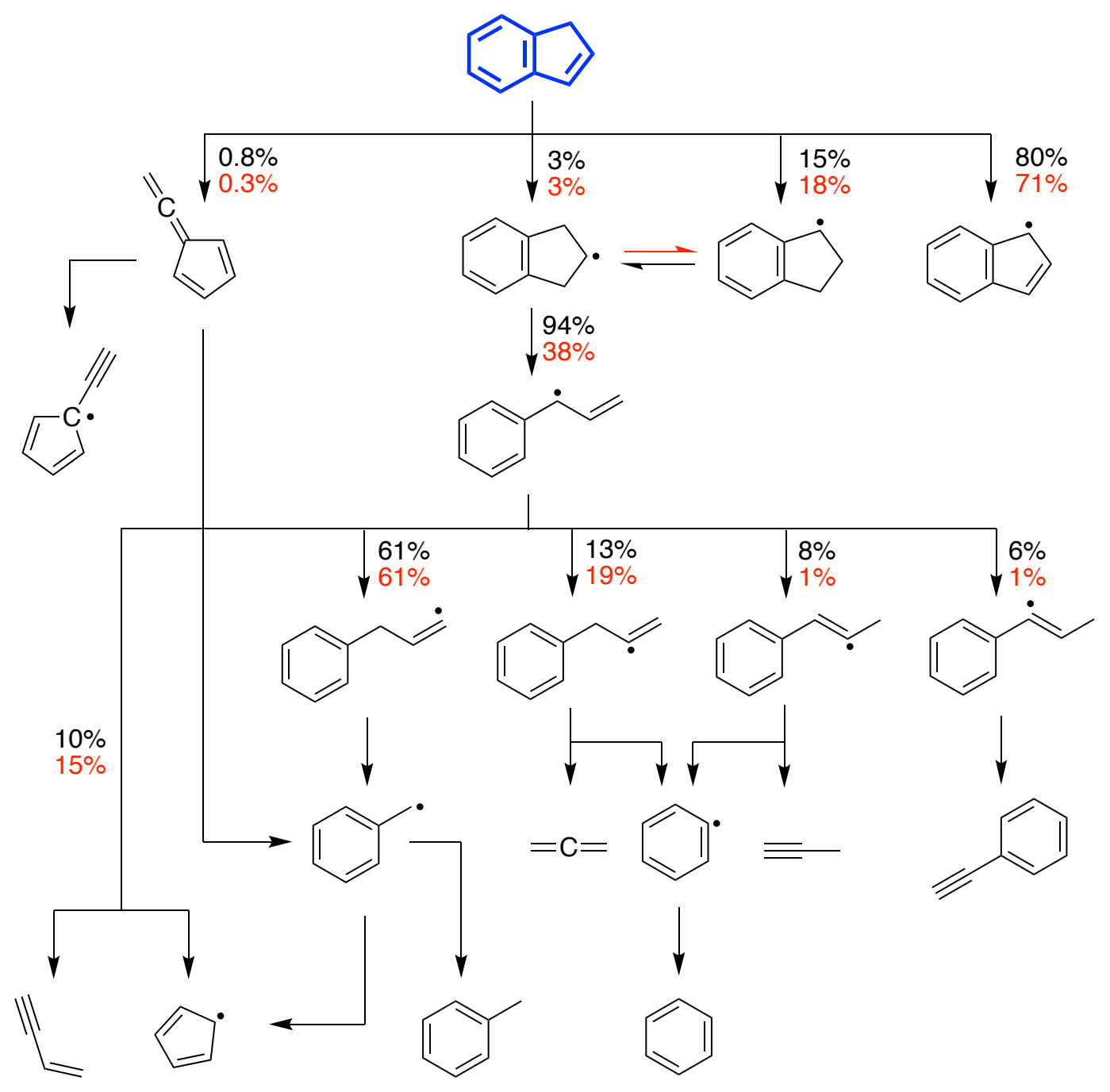

481 Figure 5 Reaction flux analysis of indene decomposition at 17.5\% conversion. Arrows indicate reaction steps; the numbers indicate the conversion ratios of each step at 30 (black) and 760 Torr (red).

Indanyl and 5-vinylidene-1,3-cyclopentadiene $\left(\mathrm{C}_{7} \mathrm{H}_{6}\right)$ are minor indene consumption products.

Rate of production (ROP) analysis in Fig. 5 shows that two indanyl radicals account for $\sim 20 \%$ of the

total carbon flux from indene, where the contribution of each channel does not change much as

487 pressure increases. Unimolecular decomposition yielding $\mathrm{C}_{7} \mathrm{H}_{6}$ contributes less than $1 \%$ to the total 488 indene consumption.

489 Sensitivity analysis for indene consumption is shown in Fig. 6. The mole fraction of indene is 490 sensitive to the high-pressure limit of R1 at both pressures. The low-pressure limit of R1 is more 491 sensitive at 30 Torr, while R2 is more sensitive at 760 Torr. H-addition reaction (R3) plays a negative 
492 effect on indene decomposition at higher pressure since it has a competition with R2 for H-atom 493 consumption.

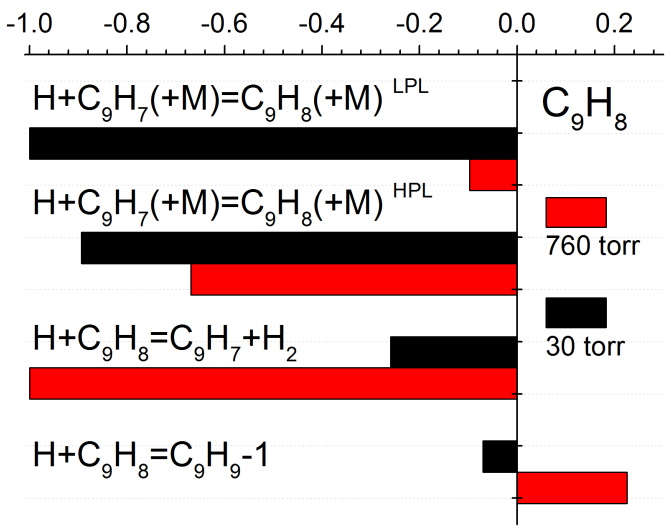

495 Figure 6 Normalized sensitivity coefficient of indene consumption, performed at an indene conversion of $17.5 \%$ at 30 and 760 Torr. Only the most sensitive four reactions are listed. HPL and LPL are high- and low-pressure limits of R1.

\subsubsection{Decomposition of $C_{9}$ intermediates}

ROP analysis (performed at $17.5 \%$ indene conversion) illustrates that indenyl decomposition is 500 negligible, though three unimolecular dissociation pathways are included in the present model. Indenyl 501 combination channels consumes $71 \%$ and $98 \%$ at 30 and 760 Torr, respectively. It should also be noted 502 that the rate constants of indenyl dissociation reactions are estimated in literature models $[39,56,57]$. 503 However, these reactions may impact PAH formation through the carbon flux competition of indenyl. 504 Therefore, accurate quantum calculations of indenyl decomposition are required to adequately 505 illustrate the kinetics of indenyl radical.

As shown in Fig. 5, 2-indanyl $\left(\mathrm{C}_{9} \mathrm{H}_{9}{ }^{2}\right)$, isomerized from 1-indanyl $\left(\mathrm{C}_{9} \mathrm{H}_{9}{ }^{1}\right)$, later opens its five507 member ring to form phenyl-allyl $\left(\mathrm{C}_{6} \mathrm{H}_{5} \mathrm{C}_{3} \mathrm{H}_{4}\right)$. At 30 Torr, indanyl mainly isomerizes to phenyl-allyl. 508 When reaction pressure raises, more indanyl reacts with indenyl and goes back to indene. Further 509 isomerization and decomposition of phenyl-allyl contributes to benzyl $(\sim 60 \%)$, phenyl $(\sim 20 \%)$, 510 cyclopentadienyl $(<15 \%)$ and phenyl-acetylene $(<6 \%)$. They are precursors of toluene, 511 cyclopentadiene and benzene. Model predictions of these species are presented in Fig. 1 and 2. 


\subsubsection{Formation of small intermediates}

514 Small intermediates, particularly $\mathrm{C}_{2}-\mathrm{C}_{5}$ hydrocarbons, are formed through various pathways via 515 multi-elementary reaction steps. 5-Vinylidene-1,3-cyclopentadiene formed via the unimolecular 516 decomposition of indene may lead to $\mathrm{C}_{7} \mathrm{H}_{7}$ or $\mathrm{C}_{7} \mathrm{H}_{5}$ radicals. $\mathrm{C}_{7} \mathrm{H}_{5}$ radical is mostly formed by the 517 decomposition of $\mathrm{C}_{7} \mathrm{H}_{6}$; it further decomposes to $\mathrm{C}_{4} \mathrm{H}_{2}, \mathrm{C}_{3} \mathrm{H}_{3}$ and $\mathrm{C}_{2} \mathrm{H}_{2}$ [108] or contributes in PAH 518 formation via combination reactions. Isomerization and dissociation of phenyl-allyl impact the 519 formation of small intermediates, such as propargyl, allene, propyne, acetylene and vinyl-acetylene. 520 Propargyl combines with cyclopentadienyl to form styrene which is a major formation pathway of 521 styrene at 760 Torr. At 30 Torr, styrene is mainly formed via a complicated H-addition pathway from 522 indanyl where phenyl-allyl and phenyl-propyl radicals are key intermediates in this process.

Model predictions of these small species are shown in Fig. 2. Only the data at 30 Torr are presented

524 for active species, such as $\mathrm{C}_{7} \mathrm{H}_{6}, \mathrm{C}_{7} \mathrm{H}_{5}, \mathrm{C}_{5} \mathrm{H}_{5}$ and $\mathrm{C}_{3} \mathrm{H}_{3}$, since the concentration of these species is too 525 low to be detected at 760 Torr. The mole fractions of small intermediates, such as allene, propyne, 526 vinyl-acetylene and butadiyne, decrease as the pressure increases. It is because the formation of these 527 species is more or less controlled by the isomerization of indanyl to phenyl-allyl, as shown in Fig. 5

\section{5.1.4. Formation of common PAHs}

Although the dominant contributor to PAH growth would be indenyl radical (Sections 5.1.4 and 5.1.5), we still want to know if other cyclopentadienyl-like or benzyl-like radicals have any 531 contribution in this process (See Section 5.1.6). One point that needs to be clarified for the experiments 532 of this work is that the mole fractions of PAHs are not calculated for every isomer due to their close 533 ionization energies. As introduced in the experimental method Section $2[68,80]$, additional 534 measurements need to be performed at photon energies between the ionization energies of every two 535 neighboring isomers. Due to the close ionization energies between isomers, the photon energy applied 536 in the measurement would be very close to the ionization energy of the target isomer. In this case, the 537 cross-section of this target isomer at the applied photon energy is very small, so that its signal would 538 be easily influenced by the uncertainties of its isomers with larger cross sections. In general, this 
treatment may introduce large random uncertainty in the mole fraction values through measurement

540 and mathematical process. Therefore, we instead decided to use one summed mole fraction of all PAH

541 isomers with the same chemical formula when comparing with modelling results.

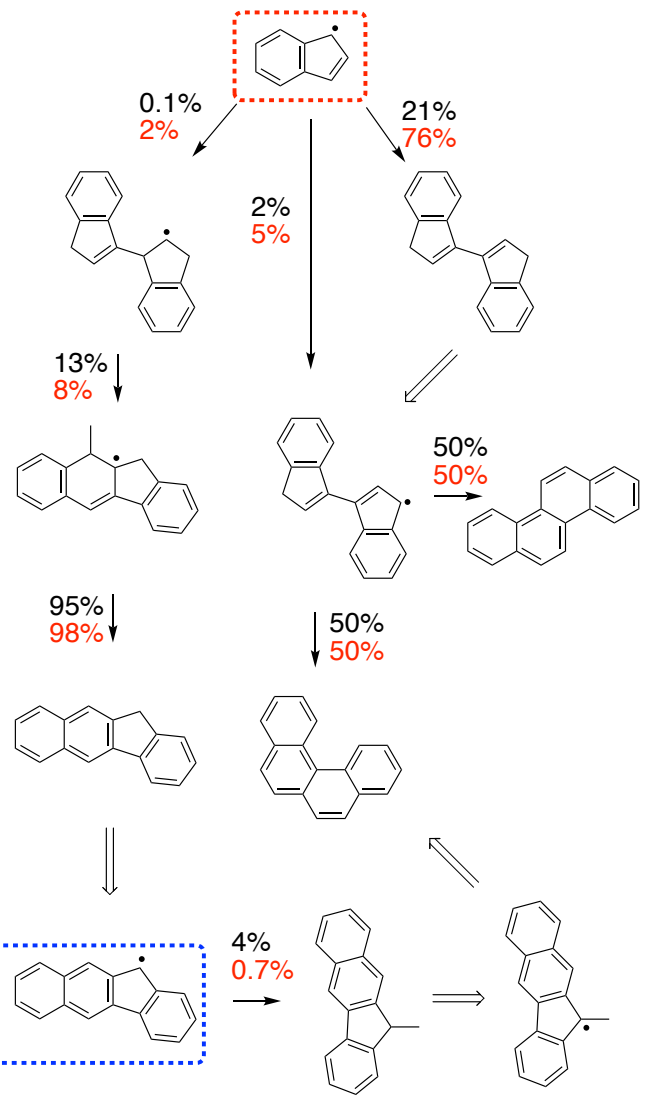

543 Figure 7 ROP analysis performed for $\mathrm{C}_{17}$ and $\mathrm{C}_{18}$ species at $70 \%$ indene conversion. The blue frame highlights larger RSR generated by indenyl radical. Numbers beside the arrows are the contributions of this reaction step in the consumption of the precursors. Black and red colors separate 30 and 760 Torr conditions, respectively. Bold arrows indicate those reaction steps that consume $>99.5 \%$ of the precursors.

$549 \mathrm{C}_{18} \mathrm{H}_{14}, \mathrm{C}_{18} \mathrm{H}_{12}, \mathrm{C}_{16} \mathrm{H}_{10}, \mathrm{C}_{14} \mathrm{H}_{10}, \mathrm{C}_{12} \mathrm{H}_{8}$ and $\mathrm{C}_{10} \mathrm{H}_{8}$. Figure $3 \mathrm{a}$ shows the model predictions of $\mathrm{C}_{18} \mathrm{H}_{14}$ 550 where the measured mole fractions are calculated with the PICS of 3,3-bi-indene. The combination of 551 two indenyl radicals is one of the most favorable reactions for indenyl. It consumes $21 \%$ and $76 \%$ of 552 indenyl at 30 and 760 Torr, respectively, as shown in Fig. 7 ROP analysis at 70\% indene conversion. 553 It forms bi-indene isomers [63] which are represented by 3,3-bi-indene in the present model. 5- and 554 11-methyl-benzofluorene are minor $\mathrm{C}_{18} \mathrm{H}_{14}$ isomers. Further reactions of $\mathrm{C}_{18} \mathrm{H}_{14}$ yield $\mathrm{C}_{18} \mathrm{H}_{12}$, which 
can be chrysene, benzo-phenanthrene and triphenylene $[10,61,63,65,109]$. Model prediction shows

556 that the quantities of chrysene and benzo-phenanthrene are comparable while that of triphenylene is

557 smaller (chrysene 62\%, benzo-phenanthrene 33\%, and triphenylene $5 \%$ at $\sim 1300 \mathrm{~K}, 760$ Torr).

558 Sensitivity analysis is performed at the temperature of $70 \%$ indene conversion at both pressures, as

559 shown in Fig. 8. Besides the formation reactions (R88 for 3,3-bi-indene and R95 for chrysene), both

560 species are sensitive to R99. It may be because R88 and R99 are two main branching pathways

561 competing for the consumption of indenyl.

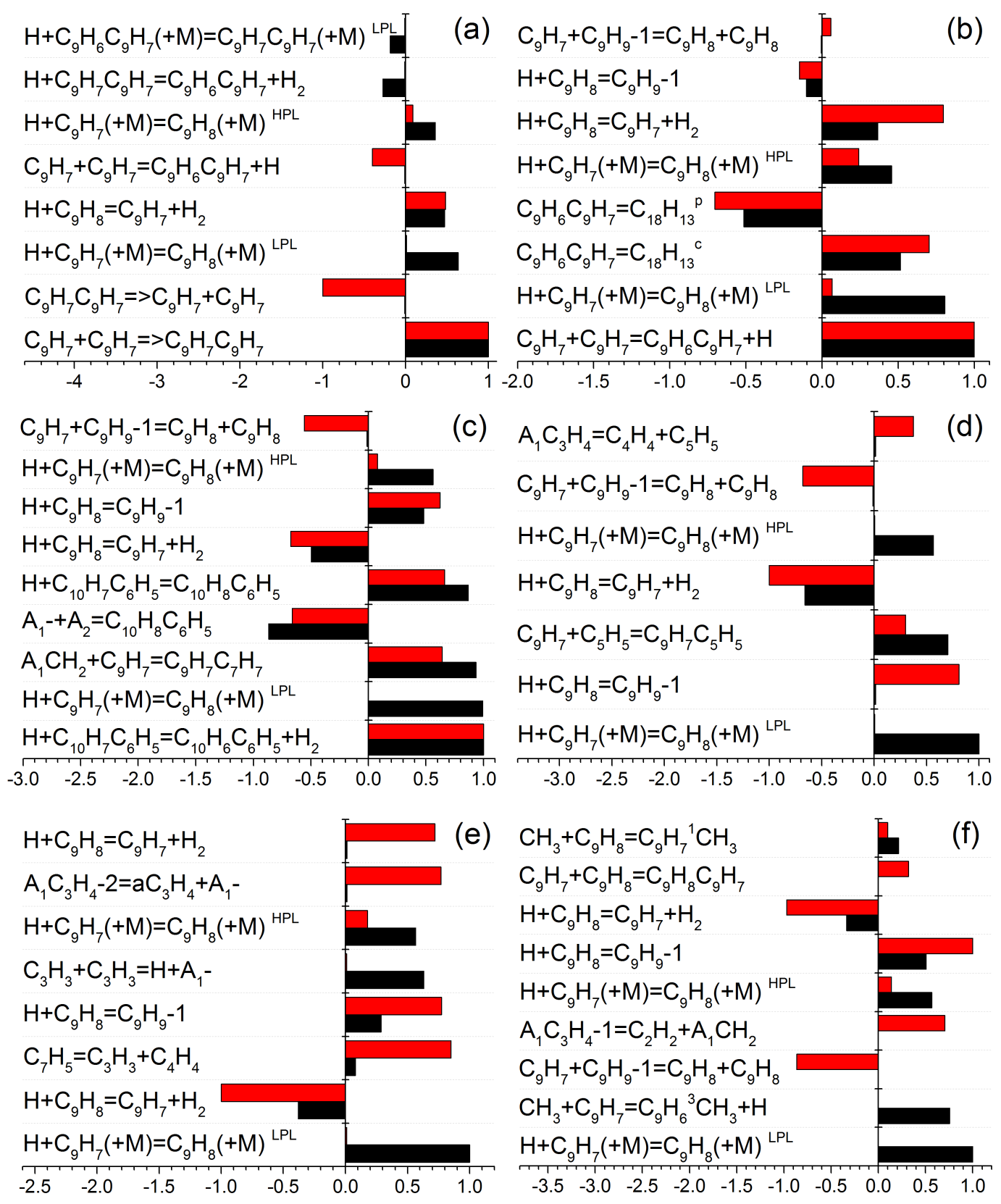


Figure 8 Sensitivity analysis performed at 70\% indene conversion on the selected PAH species: (a) 3,3-bi-indene, (b) chrysene, (c) fluoranthene, (d) phenanthrene, (e) acenaphthylene and (f) naphthalene. Black and red bars indicate 30 and 760 Torr conditions, respectively.

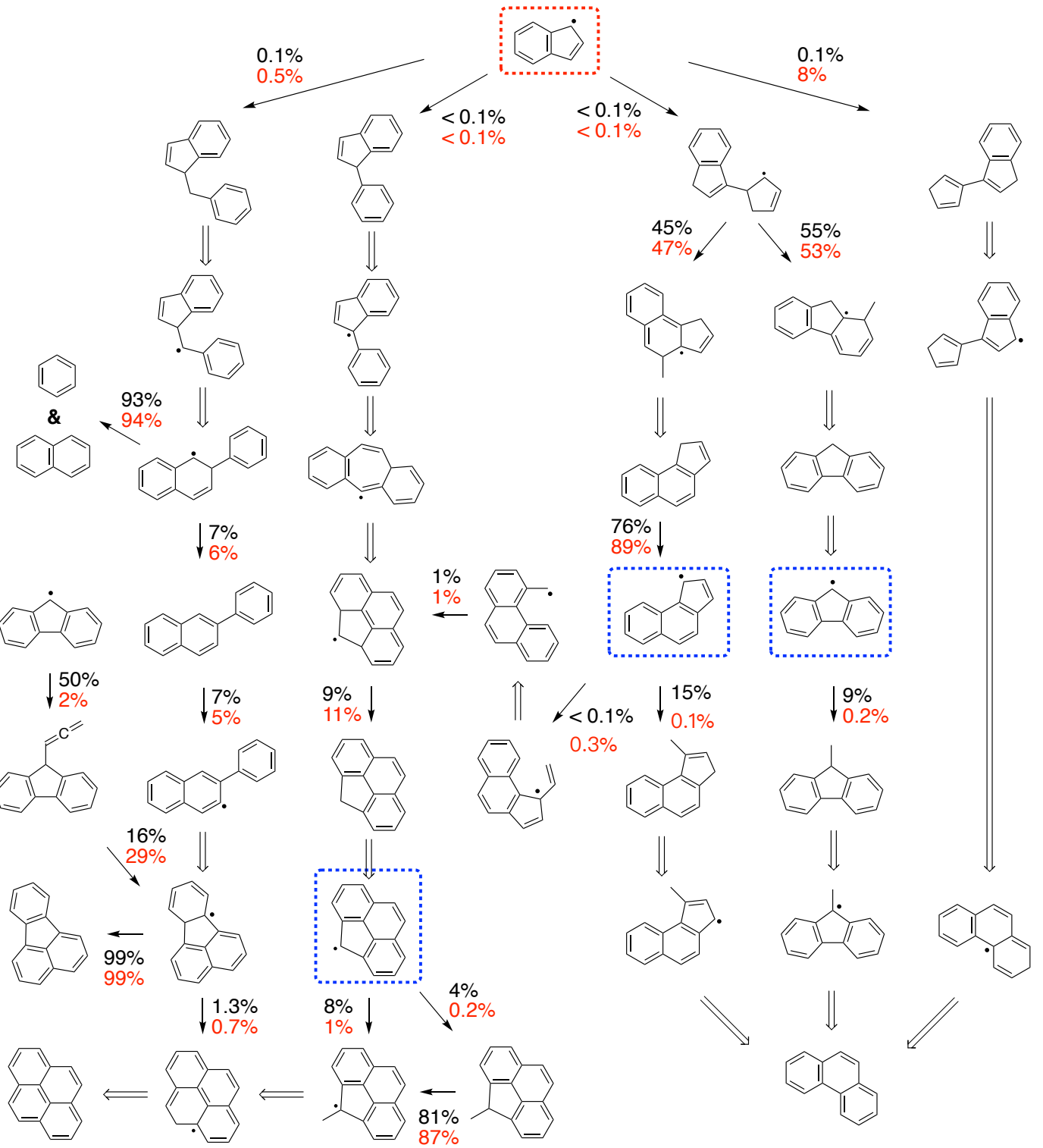

Figure 9 ROP analysis performed for $\mathrm{C}_{13}-\mathrm{C}_{16}$ species at $70 \%$ indene conversion. The blue frames highlight the larger RSRs generated by indenyl radical. Numbers beside the arrows are the contributions of this reaction step in the consumption of the precursors. Black and red colors separate 30 and 760 Torr conditions, respectively. Bold arrows indicate those reaction steps that consume $>99.5 \%$ of the precursors.

For $\mathrm{C}_{16} \mathrm{H}_{10}$ species, ROP analysis in Fig. 9 shows that indenyl reactions are the main formation source for fluoranthene and pyrene in all cases. The contributions from the reaction of benz-indenyl + 574 propargyl to pyrene and fluorenyl + propargyl to fluoranthene are much smaller, and the HACA route 
575 (phenanthrenyl + acetylene) is negligible. Sensitivity analysis of fluoranthene in Fig. 8c shows that not 576 only the combination of indenyl and benzyl (R63), but also the H-abstraction of phenyl-naphthalene 577 (R69) and $\beta$-scission of $\mathrm{C}-\mathrm{H}$ bond of $\mathrm{C}_{10} \mathrm{H}_{8} \mathrm{C}_{6} \mathrm{H}_{5}$ (R67) are rate controling reactions of $\mathrm{C}_{16} \mathrm{H}_{10}$ species. 578 R67 is a branching channel of this pathway. It competes with the $\mathrm{C}-\mathrm{C}$ bond fission of $\mathrm{C}_{10} \mathrm{H}_{8} \mathrm{C}_{6} \mathrm{H}_{5}$ for 579 naphthalene and phenyl. ROP analysis shows that R67 has a conversion ratio only around 7\%. $\mathrm{C}_{14} \mathrm{H}_{10}$ is calibrated as phenanthrene in the experiments and it has similar mole fraction to $\mathrm{C}_{16} \mathrm{H}_{10}$, 581 shown in Fig. 3. Its dominant formation pathway is the bimolecular reaction of indenyl and 582 cyclopentadienyl (R46), which consumes $8 \%$ of indenyl, as shown in Fig. 9. Sensitivity analysis in 583 Fig. 8d also shows that R46 is the rate controlling step of phenanthrene formation pathways. The 584 combination of phenyl + phenylacetylene and the decomposition of $\mathrm{C}_{13} \mathrm{H}_{9} \mathrm{CH}_{2}$ radicals (benzindenyl585 methyl or fluorenyl-methyl) have minor contributions. Another sensitive reaction is the decomposition 586 of phenyl-allyl radical $\left(\mathrm{A}_{1} \mathrm{C}_{3} \mathrm{H}_{4}\right)$, which is a source of cyclopentadienyl radical. $[40,110,111]$. Both of these may be formed by the addition of acetylene on naphthyl radical, which are competing HACA channels. When comparing their contributions to indenyl routes, HACA routes are minor. The reaction of indenyl and propargyl (R27) forms more $\mathrm{C}_{12} \mathrm{H}_{8}$ species $(>80 \%)$. Branching 591 channels of R27 and R35 lead to the formation of acenaphthylene and vinyl-naphthalene, whose ratios 592 are shown in Fig. 10. Sensitivity analysis of acenaphthylene in Fig. 8e illustrates that the formation 593 efficiency of propargyl significantly influences its concentration.

$\mathrm{C}_{10} \mathrm{H}_{8}$ is the summation of naphthalene, azulene and methylene-indene, which is calibrated as naphthalene in this experiments. $\mathrm{C}_{10} \mathrm{H}_{8}$ has higher mole fraction than $\mathrm{C}_{16} \mathrm{H}_{10}, \mathrm{C}_{14} \mathrm{H}_{10}$ and $\mathrm{C}_{12} \mathrm{H}_{8}$ but 596 less than $\mathrm{C}_{18} \mathrm{H}_{12}$, as shown in Fig. 3. ROP analyses in Figs. 9 and 10 indicate that the main naphthalene 597 formation pathway changes from methyl addition on indenyl to the decomposition of $\mathrm{C}_{10} \mathrm{H}_{8} \mathrm{C}_{6} \mathrm{H}_{5}$ 598 radical. This radical is the product of indenyl and benzyl combination via R63-R66. The former 599 pathway consumes $11 \%$ of indenyl at 30 Torr, and drops to $1 \%$ at 760 Torr. In contrast, the contribution 600 of R63 increases 5 times with pressure. Sensitivity analysis of naphthalene also reflects this 

Torr.

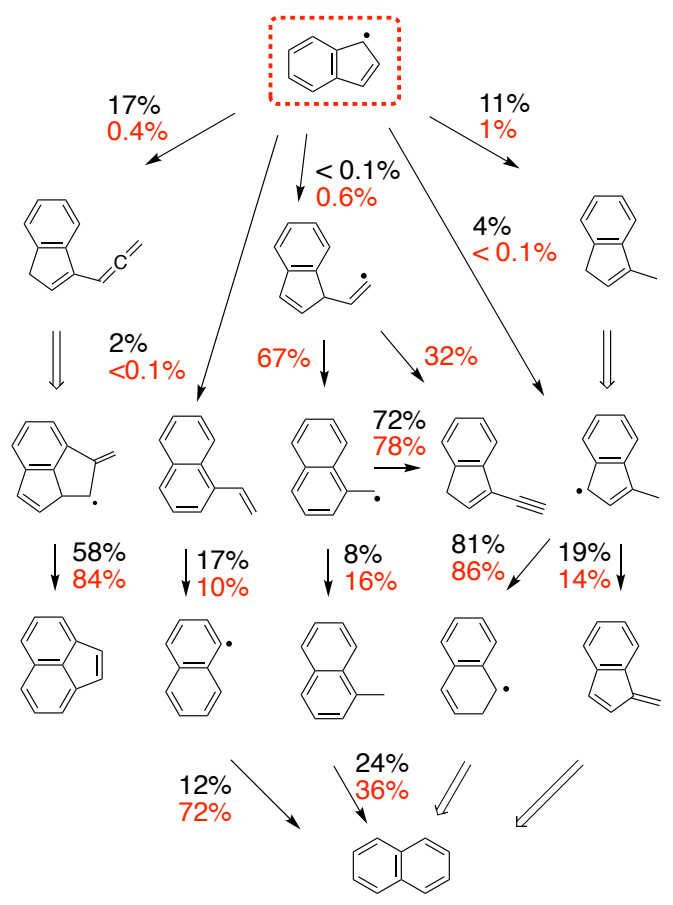

604 Figure 10 ROP analysis performed for $\mathrm{C}_{10}-\mathrm{C}_{12}$ species at $70 \%$ indene conversion. The blue frame 605 highlights larger RSR generated by indenyl radical. Numbers beside the arrows are the contributions of this reaction step in the consumption of the precursors. Black and red colors separate 30 and 760 Torr conditions, respectively. Bold arrows indicate those reaction steps that consume $>99.5 \%$ of the precursors.

611 On one hand, indenyl is mainly formed via R1 at low pressure, while on the other hand, R1 provides

$612 \mathrm{H}$ atoms that enhance the formation of indanyl. It leads to small intermediates which are the source for 613 addition reactions to PAHs. R2 has positive sensitivity for 3,3-bi-indene and chrysene, but negative 614 for fluoranthene, phenanthrene, acenaphthylene and naphthalene. This is because H-abstraction of 615 indene (R2) is competing with $\mathrm{H}$-addition of indene (R3). The formation of latter four PAHs not only 616 needs indenyl but also corresponding small intermediates. 


\subsubsection{Uncommon PAHs formation}

Figure 4 shows some PAH species that are not commonly included in previous modeling studies.

621 They are normally odd C-number species that are ignored in traditional HACA mechanism but are 622 considered to be critical in this study via radical combination PAH pathways. $\mathrm{C}_{17} \mathrm{H}_{12}$ is is speculated 623 to be benzo-fluorene [65], and its mole fraction increases significantly with the pressure. $\mathrm{m} / \mathrm{z}=190$ is $624 \mathrm{C}_{15} \mathrm{H}_{10}$, and its molecular structure is proposed to be $4 H$-cyclopenta[def]phenanthrene in literature $625[112,113]$. ROP analysis shows that its formation consumes less than $0.1 \%$ of indenyl. Since bi-indene 626 is detected in this study, indenyl-cyclopentadiene, having similar reactivity, is also detectable by the 627 same technique. It could be a candidate of $\mathrm{C}_{14} \mathrm{H}_{12}$ species. Other possible $\mathrm{C}_{14} \mathrm{H}_{12}$ isomers are methyl628 fluorene and methyl-benzindene. Quantitative modelling results show that the mole fractions of the 629 latter two isomers are higher than that of indenyl-cyclopentadiene. The reactions of $\mathrm{C}_{9} \mathrm{H}_{8}+\mathrm{C}_{5} \mathrm{H}_{5}$ and $630 \mathrm{C}_{9} \mathrm{H}_{7}+\mathrm{C}_{5} \mathrm{H}_{6}$ are not favored to produce indenyl-cyclopentadiene, but are more likely to form fluorene 631 and benz-indene $\left(\mathrm{C}_{13} \mathrm{H}_{10}\right)$, as shown by the ROP analysis in Fig. 9. We also observe from ROP analysis 632 that $\mathrm{C}_{9} \mathrm{H}_{7}+\mathrm{iC}_{4} \mathrm{H}_{3}$ reaction has considerable contribution in fluorene formation. $\mathrm{C}_{11} \mathrm{H}_{8}$ is calibrated as 633 ethynyl-indene in the experiments $[53,96,114]$. In general, the proposed formation pathways in the present model reproduce the measured mole fraction quite well at both pressures, as shown in Fig. 4 . formation of $\mathrm{C}_{10} \mathrm{H}_{10}$ in Fig. 4f. It could be because of the unknown methyl-indene formation pathways or the under-prediction of methyl radical at temperatures around $1100 \mathrm{~K}$. An important source of 638 methyl radical is the reaction of indenyl and indene. It produces benzo-fluorene while releasing a 639 methyl radicals. However, this part of the present model is analogized to the kinetics of 640 cyclopentadienyl radical, which may introduce large uncertainty to methyl prediction. Further detailed 641 calculations of this scheme at the potential energy surface of $\mathrm{C}_{18} \mathrm{H}_{15}$ may reveal the kinetics of benzo642 fluorene and methyl-indene. 


\subsubsection{PAHs grown from other RSR radicals}

Generally, PAH species can be divided into different aromatic groups: monocyclic, bicyclic,

647 tricyclic, etc. Five-member-ring RSRs can be formed in every aromatic group as highlighted by the 648 blue rectangles in Figs. 7 and 9. The smallest five-member-ring RSR is cyclopentadienyl radical. 649 Indenyl radical studied in this work is a bicyclic five-member-ring RSR. With the addition of other 650 five-member-ring species, the five-member-ring RSRs could grow bigger through self-incentive 651 reaction processes. For example, indene is yielded from cyclopentadienyl radical $[49,86,115]$ while 652 fluorene and benz-indene are yielded via $\mathrm{C}_{9} \mathrm{H}_{7}+\mathrm{C}_{5} \mathrm{H}_{6}$ or $\mathrm{C}_{5} \mathrm{H}_{5}+\mathrm{C}_{9} \mathrm{H}_{8}$ [50]. Therefore, similar 653 reactions are also possible for fluorenyl, benz-indenyl, benzo-fluorenyl and even larger five-member654 ring RSRs. This efficient PAH growth moiety agrees with the radical chain reaction, recommended recently by Johansson et al. [59].

Besides the five-member-ring RSRs, the contribution of other RSRs is not so remarkable in indene pyrolysis, which can be ascribed to the fuel selectivity of PAH growth pathways. Propargyl, benzyl, 658 tropyl, vinyl-cyclopentadienyl, and other large RSRs with similar structures are potential contributors. 659 For example, in Figs. 9 and 10, the addition reactions of propargyl on indenyl and fluorenyl are quite 660 efficient to build larger PAHs. Detailed chemical kinetics for some of these were investigated in 661 previous studies [31,42,45,47,54,85,116-120]; however, there is a lack of knowledge about more other 662 RSRs, especially large ones, which may be comparatively as important as indenyl in PAH growth.

\subsection{Indene pyrolysis in shock tube}

Laskin and Liftshitz studied indene pyrolysis in a shock tube at a pressure of 3.7 atm over a temperature range of $1150-1900 \mathrm{~K}$ [62]. Quantitative species measurements were provided by gas

667 chromatography (GC). We adopted this dataset to validate the present model. Comparisons between 668 experimental data and simulations are presented in Figs. 11 and 12. Note that simulated temperatures 669 were shifted by $80 \mathrm{~K}$ to match indene conversion [121,122] in order to compare experimental and 670 simulated branching fractions for an equivalent conversion. The present model adequately captures 
the mole fractions of most species; only a few species, like naphthalene, methylene-indene and benzene, are slightly over-predicted. Predictions by literature models are also compared in Figs. 11 and 12 .

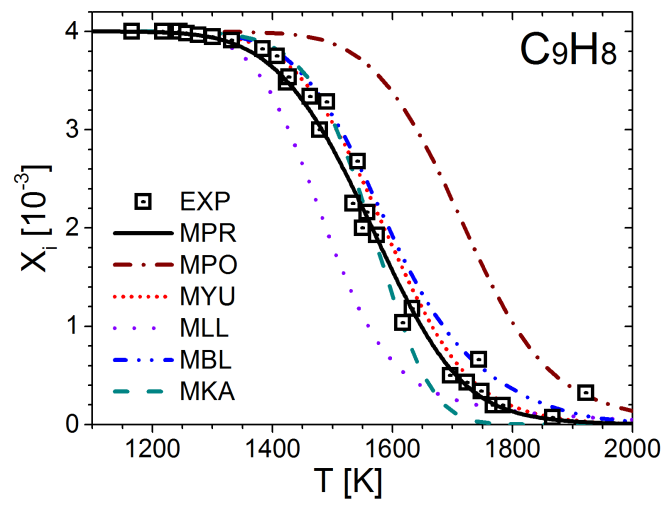

675

676

677

678

679

680

Figure 11 Modeling results of indene decomposition in a shock tube; experimental data are taken from [62]. Simulated results were shifted by $80 \mathrm{~K}$ according to the literature [121,122]. MPR: present model; MPO: Polimi model [81]; MYU: Yuan et al.'s model [9]; MLL: Laurence Livermore model [58]; MBL: Blanquart et al.'s model [7]; MKA: Wang et al.'s model [8].
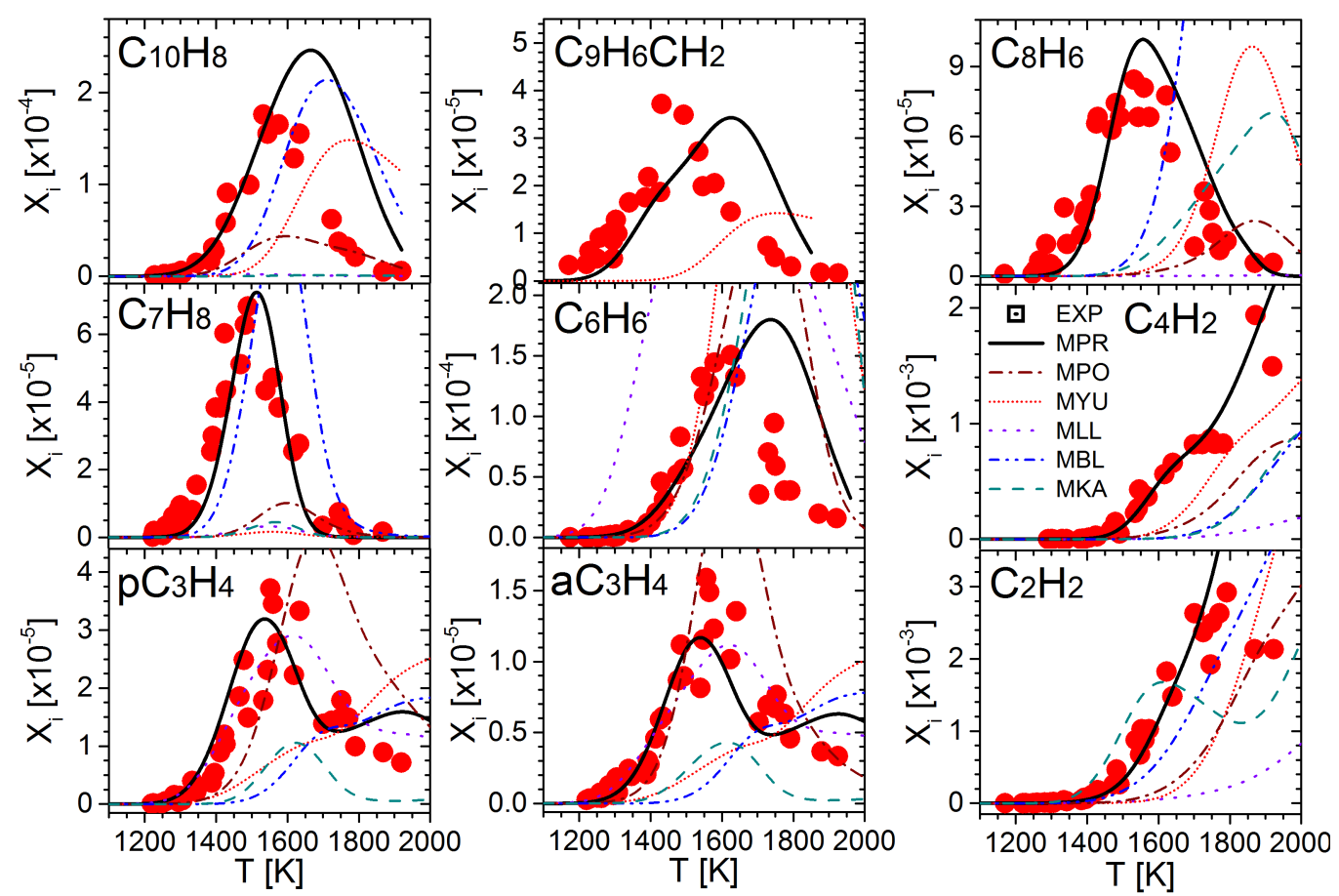

Figure 12 Predicted mole fraction profiles of the species measured in shock tube indene pyrolysis [62]. Simulated results were shifted by $80 \mathrm{~K}$ according to the literature [121,122]. MPR: present model; MPO: Polimi model [81]; MYU: Yuan et al.'s model [9]; MLL: Laurence Livermore model [58]; MBL: Blanquart et al.'s model [7]; MKA: Wang et al.'s model [8]. 


\section{Conclusions}

Pyrolysis of indene was investigated in a flow reactor at 30 and 760 Torr over the temperature range of 975 - $1450 \mathrm{~K}$ using SVUV-PIMS. A number of pyrolytic species were quantified, such as

689 free radicals, various small hydrocarbons and PAHs. Their mole fraction profiles were measured as a 690 function of reactor temperature. Based on these experimental observations, an improved kinetic model 691 was developed based on previous PAH models [7,9,10,13-15,56,57,66,77]. We adopted recent 692 theoretical progress and added some necessary reaction schemes in our model. The present model was 693 then validated against the flow reactor data from this study and datasets reported in literature [62]. By 694 performing ROP and sensitivity analysis, critical reaction pathways were identified for indene 695 pyrolysis, particularly those leading to PAH formation from indenyl radical.

Under the pyrolysis condition, indene is mainly consumed by unimolecular C-H bond dissociation

697 and $\mathrm{H}$-abstraction reactions. Both of these pathways have comparable contribution from low to 698 atmospheric pressure. Subsequent reactions of indenyl favor the PAH channels, since indenyl is not 699 easy to decompose at the conditions of this study. The dominant PAH formation pathways in this study 700 are the bi-molecular addition reactions of indenyl radical with indene or a series of other intermediates. 701 PAH growth also has particular contribution from the reactions of other resonantly stabilized radicals 702 with five-member ring in their molecular structures, such as cyclopentadienyl, fluorenyl, benz-indenyl 703 and benzo-fluorenyl. Radical chain reactions provide huge passage for PAH growth form one RSR to 704 larger ones. Typically, the cyclical addition of acetylene on indenyl yields vinyl-indenyl or benz705 indenyl, while the cyclical addition of indene on indenyl yields benzo-fluorenyl and even larger RSRs, 706 although these RSRs are not detected in this study. Further PAH modeling studies should refine the 707 reaction network of other typical RSRs besides indenyl radical, which may help provide prediction of 708 not only the concentration but also the chemical and/or physical structures of PAHs and soot. 
Research reported in this publication was funded by the Office of Sponsored Research at King Abdullah University of Science and Technology (KAUST), Key Science Foundation of Higher Education of Henan (19A480002) and National Natural Science Foundation of China (91541201 and $51622605)$.

\section{References}

[1] A. Ebenstein, M. Fan, M. Greenstone, G. He, M. Zhou, New evidence on the impact of sustained exposure to air pollution on life expectancy from China's Huai River Policy, Proc. Natl. Acad. Sci. U. S. A. 114 (2017) $720 \quad 10384-10389$.

[2] I.C. Pope, R.T. Burnett, M.J. Thun, et al., Lung cancer, cardiopulmonary mortality, and long-term exposure to fine particulate air pollution, JAMA 287 (2002) 1132-1141.

[3] H. Richter, J.B. Howard, Formation of polycyclic aromatic hydrocarbons and their growth to soot - a review of chemical reaction pathways, Prog. Energy Combust. Sci. 26 (2000) 565-608.

[4] C.S. McEnally, L.D. Pfefferle, B. Atakan, K. Kohse-Hoinghaus, Studies of aromatic hydrocarbon formation mechanisms in flames: Progress towards closing the fuel gap, Prog. Energy Combust. Sci. 32 (2006) 247-294.

[5] H. Wang, Formation of nascent soot and other condensed-phase materials in flames, Proc. Combust. Inst. 33 (2011) 41-67.

[6] K. Kohse-Höinghaus, Clean combustion: Chemistry and diagnostics for a systems approach in transportation and energy conversion, Prog. Energy Combust. Sci. 65 (2018) 1-5.

[7] G. Blanquart, P. Pepiot-Desjardins, H. Pitsch, Chemical mechanism for high temperature combustion of engine relevant fuels with emphasis on soot precursors, Combust. Flame 156 (2009) 588-607.

[8] Y. Wang, A. Raj, S.H. Chung, A PAH growth mechanism and synergistic effect on PAH formation in counterflow diffusion flames, Combust. Flame 160 (2013) 1667-1676.

[9] W.H. Yuan, Y.Y. Li, P. Dagaut, J.Z. Yang, F. Qi, Investigation on the pyrolysis and oxidation of toluene over a wide range conditions. II. A comprehensive kinetic modeling study, Combust. Flame 162 (2015) 22-40.

[10] N.A. Slavinskaya, P. Frank, A modelling study of aromatic soot precursors formation in laminar methane and ethene flames, Combust. Flame 156 (2009) 1705-1722.

[11] H. Richter, W.J. Grieco, J.B. Howard, Formation mechanism of polycyclic aromatic hydrocarbons and fullerenes in premixed benzene flames, Combust. Flame 119 (1999) 1-22.

[12] N.M. Marinov, W.J. Pitz, C.K. Westbrook, M.J. Castaldi, S.M. Senkan, Modeling of aromatic and polycyclic aromatic hydrocarbon formation in premixed methane and ethane flames, Combust. Sci. Technol. 116-117 (1996) 211-287.

[13] J. Appel, H. Bockhorn, M. Frenklach, Kinetic modeling of soot formation with detailed chemistry and physics: Laminar premixed flames of C2 hydrocarbons, Combust. Flame 121 (2000) 122-136.

[14] H. Wang, M. Frenklach, A detailed kinetic modeling study of aromatics formation in laminar premixed acetylene and ethylene flames, Combust. Flame 110 (1997) 173-221. 
[15] H. Jin, A. Frassoldati, Y. Wang, X. Zhang, M. Zeng, Y. Li, F. Qi, A. Cuoci, T. Faravelli, Kinetic modeling study of benzene and PAH formation in laminar methane flames, Combust. Flame 162 (2015) 1692-1711.

[16] H. Wang, M. Frenklach, Calculations of Rate Coefficients for the Chemically Activated Reactions of Acetylene with Vinylic and Aromatic Radicals, J. Phys. Chem. 98 (1994) 11465-11489.

[17] F. Xu, K.C. Lin, G.M. Faeth, Soot formation in laminar premixed methane/oxygen flames at atmospheric pressure, Combust. Flame 115 (1998) 195-209.

[18] N.E. Sánchez, A. Callejas, Á. Millera, R. Bilbao, M.U. Alzueta, Polycyclic Aromatic Hydrocarbon (PAH) and Soot Formation in the Pyrolysis of Acetylene and Ethylene: Effect of the Reaction Temperature, Energy Fuels 26 (2012) 4823-4829.

[19] C. Saggese, N.E. Sánchez, A. Frassoldati, A. Cuoci, T. Faravelli, M.U. Alzueta, E. Ranzi, Kinetic Modeling Study of Polycyclic Aromatic Hydrocarbons and Soot Formation in Acetylene Pyrolysis, Energy Fuels 28 (2013) 1489-1501.

[20] S.A. Skeen, B. Yang, H.A. Michelsen, J.A. Miller, A. Violi, N. Hansen, Studies of laminar opposed-flow diffusion flames of acetylene at low-pressures with photoionization mass spectrometry, Proc. Combust. Inst. 34 (2013) 1067-1075.

[21] J.D. Bittner, J.B. Howard, Composition profiles and reaction mechanisms in a near-sooting premixed benzene/oxygen/argon flame, Proc. Combust. Inst. 18 (1981) 1105-1116.

[22] J. Griesheimer, K.H. Homann, Large molecules, radicals ions, and small soot particles in fuel-rich hydrocarbon flames: Part II. Aromatic radicals and intermediate PAHs in a premixed low-pressure naphthalene/oxygen/argon flame, Proc. Combust. Inst. 27 (1998) 1753-1759.

[23] R.P. Lindstedt, G. Skevis, A.R. Burgess, F.L. Dryer, Benzene formation chemistry in premixed 1,3butadiene flames, Proc. Combust. Inst. 26 (1996) 703-709.

[24] J.A. Miller, C.F. Melius, Kinetic and thermodynamic issues in the formation of aromatic compounds in flames of aliphatic fuels, Combust. Flame 91 (1992) 21-39.

[25] E.W. Diau, M.C. Lin, C.F. Melius, A theoretical study of the $\mathrm{CH}_{3}+\mathrm{C}_{2} \mathrm{H}_{2}$ reaction, J. Chem. Phys. 101 (1994) 3923-3927.

[26] A.M. Dean, Detailed kinetic modeling of autocatalysis in methane pyrolysis, J. Phys. Chem. 94 (1990) $1432-1439$.

[27] M.B. Colket, D.J. Seery, Reaction mechanisms for toluene pyrolysis, Proc. Combust. Inst. 25 (1994) 883891.

[28] C.F. Melius, M.E. Colvin, N.M. Marinov, W.J. Pitz, S.M. Senkan, Reaction mechanisms in aromatic hydrocarbon formation involving the C5H5 cyclopentadienyl moiety, Proc. Combust. Inst. 26 (1996) 685-692.

[29] N.M. Marinov, W.J. Pitz, C.K. Westbrook, A.M. Vincitore, M.J. Castaldi, S.M. Senkan, C.F. Melius, Aromatic and Polycyclic Aromatic Hydrocarbon Formation in a Laminar Premixed n-Butane Flame, Combust. Flame 114 (1998) 192-213.

[30] A. D'Anna, J.H. Kent, Aromatic formation pathways in non-premixed methane flames, Combust. Flame 132 (2003) 715-722.

[31] C.L. Rasmussen, M.S. Skjøth-Rasmussen, A.D. Jensen, P. Glarborg, Propargyl recombination: estimation of the high temperature, low pressure rate constant from flame measurements, Proc. Combust. Inst. 30 (2005) 1023-1031.

[32] N. Hansen, J.A. Miller, P.R. Westmoreland, T. Kasper, K. Kohse-Höinghaus, J. Wang, T.A. Cool, Isomerspecific combustion chemistry in allene and propyne flames, Combust. Flame 156 (2009) 2153-2164.

[33] M.R. Djokic, K.M. Van Geem, C. Cavallotti, A. Frassoldati, E. Ranzi, G.B. Marin, An experimental and kinetic modeling study of cyclopentadiene pyrolysis: First growth of polycyclic aromatic hydrocarbons, Combust. Flame 161 (2014) 2739-2751.

[34] R.K. Robinson, R.P. Lindstedt, On the chemical kinetics of cyclopentadiene oxidation, Combust. Flame 158 (2011) 666-686. 
[35] R.G. Butler, I. Glassman, Cyclopentadiene combustion in a plug flow reactor near 1150 K, Proc. Combust. Inst. 32 (2009) 395-402.

[36] A. Lifshitz, C. Tamburu, A. Suslensky, F. Dubnikova, Decomposition and ring expansion in methylcyclopentadiene: single-pulse shock tube and modeling study, Proc. Combust. Inst. 30 (2005) 1039-1047.

[37] J. Filley, J.T. McKinnon, Dimerization of cyclopentadienyl radical to produce naphthalene, Combust. Flame 124 (2001) 721-723.

[38] R.P. Lindstedt, K.A. Rizos, The formation and oxidation of aromatics in cyclopentene and methylcyclopentadiene mixtures, Proc. Combust. Inst. 29 (2002) 2291-2298.

[39] N.A. Slavinskaya, U. Riedel, S.B. Dworkin, M.J. Thomson, Detailed numerical modeling of PAH formation and growth in non-premixed ethylene and ethane flames, Combust. Flame 159 (2012) 979-995.

[40] B. Shukla, M. Koshi, Comparative study on the growth mechanisms of PAHs, Combust. Flame 158 (2011) 369-375.

[41] N. Hansen, M. Schenk, K. Moshammer, K. Kohse-Höinghaus, Investigating repetitive reaction pathways for the formation of polycyclic aromatic hydrocarbons in combustion processes, Combust. Flame 180 (2017) $250-261$.

[42] J.A. Miller, S.J. Klippenstein, The Recombination of Propargyl Radicals and Other Reactions on a $\mathrm{C}_{6} \mathrm{H}_{6}$ Potential, J. Phys. Chem. A 107 (2003) 7783-7799.

[43] V.V. Kislov, A.M. Mebel, The formation of naphthalene, azulene, and fulvalene from cyclic C-5 species in combustion: An ab Initio/RRKM study of 9-h-fulvalenyl (C5H5-C5H4) radical rearrangements, J. Phys. Chem. A 111 (2007) 9532-9543.

[44] A.M. Mebel, V.V. Kislov, Can the C5H5 + C5H5 $\rightarrow$ C10H10 $\rightarrow$ C10H9 + H/C10H8 + H2 Reaction Produce Naphthalene? An Ab Initio/RRKM Study, J. Phys. Chem. A 113 (2009) 9825-9833.

[45] A. Matsugi, A. Miyoshi, Computational study on the recombination reaction between benzyl and propargyl radicals, Int. J. Chem. Kinet. 44 (2012) 206-218.

[46] C. Cavallotti, D. Polino, On the kinetics of the $\mathrm{C}_{5} \mathrm{H}_{5}+\mathrm{C}_{5} \mathrm{H}_{5}$ reaction, Proc. Combust. Inst. 34 (2013) 557564 .

[47] S. Sinha, R.K. Rahman, A. Raj, On the role of resonantly stabilized radicals in polycyclic aromatic hydrocarbon $(\mathrm{PAH})$ formation: pyrene and fluoranthene formation from benzyl-indenyl addition, Phys. Chem. Chem. Phys. 19 (2017) 19262-19278.

[48] A.E. Long, S.S. Merchant, A.G. Vandeputte, H.-H. Carstensen, A.J. Vervust, G.B. Marin, K.M. Van Geem, W.H. Green, Pressure dependent kinetic analysis of pathways to naphthalene from cyclopentadienyl recombination, Combust. Flame 187 (2018) 247-256.

[49] D. Wang, A. Violi, D.H. Kim, J.A. Mullholland, Formation of Naphthalene, Indene, and Benzene from Cyclopentadiene Pyrolysis: A DFT Study, J. Phys. Chem. A 110 (2006) 4719-4725.

[50] J.A. Mulholland, M. Lu, D.-H. Kim, Pyrolytic growth of polycyclic aromatic hydrocarbons by cyclopentadienyl moieties, Proc. Combust. Inst. 28 (2000) 2593-2599.

[51] S. Sharma, W.H. Green, Computed Rate Coefficients and Product Yields for c-C5H5 + CH3 $\rightarrow$ Products, J. Phys. Chem. A 113 (2009) 8871-8882.

[52] R.S. Tranter, S.J. Klippenstein, L.B. Harding, B.R. Giri, X.L. Yang, J.H. Kiefer, Experimental and Theoretical Investigation of the Self-Reaction of Phenyl Radicals, J. Phys. Chem. A 114 (2010) 8240-8261.

[53] A.M. Mebel, A. Landera, R.I. Kaiser, Formation Mechanisms of Naphthalene and Indene: From the Interstellar Medium to Combustion Flames, J. Phys. Chem. A 121 (2017) 901-926.

[54] A. Raj, M.J. Al Rashidi, S.H. Chung, S.M. Sarathy, PAH Growth Initiated by Propargyl Addition: Mechanism Development and Computational Kinetics, J. Phys. Chem. A 118 (2014) 2865-2885.

[55] W. Yuan, Y. Li, P. Dagaut, J. Yang, F. Qi, Experimental and kinetic modeling study of styrene combustion, Combust. Flame 162 (2015) 1868-1883. 
[56] W.H. Yuan, Y.Y. Li, P. Dagaut, J.Z. Yang, F. Qi, Investigation on the pyrolysis and oxidation of toluene over a wide range conditions. I. Flow reactor pyrolysis and jet stirred reactor oxidation, Combust. Flame 162 (2015) 3-21.

[57] A. Matsugi, A. Miyoshi, Modeling of two- and three-ring aromatics formation in the pyrolysis of toluene, Proc. Combust. Inst. 34 (2013) 269-277.

[58] H. Nakamura, D. Darcy, M. Mehl, C.J. Tobin, W.K. Metcalfe, W.J. Pitz, C.K. Westbrook, H.J. Curran, An experimental and modeling study of shock tube and rapid compression machine ignition of n-butylbenzene/air mixtures, Combust. Flame 161 (2014) 49-64.

[59] K.O. Johansson, M.P. Head-Gordon, P.E. Schrader, K.R. Wilson, H.A. Michelsen, Resonance-stabilized hydrocarbon-radical chain reactions may explain soot inception and growth, Science 361 (2018) 997-1000.

[60] H. Jin, G. Wang, Y. Wang, X. Zhang, Y. Li, Z. Zhou, J. Yang, F. Qi, Experimental and kinetic modeling study of laminar coflow diffusion methane flames doped with iso -butanol, Proc. Combust. Inst. 36 (2017) 12591267.

[61] A. Ajaz, A.C. Voukides, K.J. Cahill, R. Thamatam, S.L. Skraba-Joiner, R.P. Johnson, Microwave Flash Pyrolysis: C9H8 Interconversions and Dimerisations, Aust. J. Chem. 67 (2014) 1301-1308.

[62] A. Laskin, A. Lifshitz, Thermal decomposition of indene. Experimental results and kinetic modeling, Proc. Combust. Inst. 27 (1998) 313-320.

[63] C. Wentrup, H.-W. Winter, D. Kvaskoff, C9H8 Pyrolysis. o-Tolylacetylene, Indene, 1-Indenyl, and Biindenyls and the Mechanism of Indene Pyrolysis, J. Phys. Chem. A 119 (2015) 6370-6376.

[64] C. Cavallotti, S. Mancarella, R. Rota, S. Carrà, Conversion of C5 into C6 Cyclic Species through the Formation of C7 Intermediates $\dagger$, J. Phys. Chem. A 111 (2007) 3959-3969.

[65] M. Lu, J.A. Mulholland, Aromatic hydrocarbon growth from indene, Chemosphere 42 (2001) 625-633.

[66] A. Lifshitz, C. Tamburu, A. Suslensky, F. Dubnikova, Decomposition, Isomerization, and Ring Expansion in 2-Methylindene: Single-pulse Shock Tube and Modeling Study, J. Phys. Chem. A 108 (2004) 3430-3438.

[67] G.M. Badger, R.W.L. Kimber, The formation of aromatic hydrocarbons at high temperatures Part VII The pyrolysis of indene, J. Chem. Soc., (1960) 2746-2749.

[68] F. Qi, Combustion chemistry probed by synchrotron VUV photoionization mass spectrometry, Proc. Combust. Inst. 34 (2013) 33-63.

[69] Z. Zhou, X. Du, J. Yang, Y. Wang, C. Li, S. Wei, L. Du, Y. Li, F. Qi, Q. Wang, The vacuum ultraviolet beamline/endstations at NSRL dedicated to combustion research, J. Synchrot. Radiat. 23 (2016) 1035-1045.

[70] J. Cai, W. Yuan, L. Ye, Z. Cheng, Y. Wang, L. Zhang, F. Zhang, Y. Li, F. Qi, Experimental and kinetic modeling study of 2-butanol pyrolysis and combustion, Combust. Flame 160 (2013) 1939-1957.

[71] Y. Zhao, D.G. Truhlar, The M06 suite of density functionals for main group thermochemistry, thermochemical kinetics, noncovalent interactions, excited states, and transition elements: two new functionals and systematic testing of four M06-class functionals and 12 other functionals, Theor. Chem. Acc. 120 (2008) 215-241.

[72] B.J. Lynch, Y. Zhao, D.G. Truhlar, Effectiveness of Diffuse Basis Functions for Calculating Relative Energies by Density Functional Theory, J. Phys. Chem. A 107 (2003) 1384-1388.

[73] R. Krishnan, J.S. Binkley, R. Seeger, J.A. Pople, Self-consistent molecular orbital methods. XX. A basis set for correlated wave functions, J. Chem. Phys. 72 (1980) 650-654.

[74] M.J.T. Frisch, G. W.; Schlegel, H. B.; Scuseria, G. E.; M.A.C. Robb, J. R.; Scalmani, G.; Barone, V.; Mennucci,, G.A.N. B.; Petersson, H.; Caricato, M.; Li, X.; Hratchian, H., A.F.B. P.; Izmaylov, J.; Zheng, G.; Sonnenberg, J. L.; Hada, M.; M.T. Ehara, K.; Fukuda, R.; Hasegawa, J.; Ishida, M.; Nakajima,, Y.K. T.; Honda, O.; Nakai, H.; Vreven, T.; Montgomery, J. A., Jr.; J.E.O. Peralta, F.; Bearpark, M.; Heyd, J. J.; Brothers, E.; Kudin,, V.N.K. K. N.; Staroverov, R.; Normand, J.; Raghavachari, K.; A.B. Rendell, J. C.; Iyengar, S. S.; Tomasi, J.; Cossi, M.; Rega,, J.M.K. N.; Millam, M.; Knox, J. E.; Cross, J. B.; Bakken, V.; C.J. Adamo, J.; Gomperts, R.; Stratmann, R. E.; Yazyev, O.; A.J.C. Austin, R.; Pomelli, C.; Ochterski, J. W.; Martin, R. L.; K.Z. Morokuma, V. G.; Voth, G. A.; Salvador, P.; J.J.D. Dannenberg, S.; Daniels, A. D.; Farkas, O.; J.B.O. Foresman, J. V.; Cioslowski, J.; Fox, D. J. Gaussian 09, revision C.01; Gaussian, Inc.: Wallingford, CT, 2009. 
[75] Y. Zhao, R. Peverati, K. Tang, S. Luo, H.S. Yu, X. He, D.G. Truhlar, Minnesota Gaussian Functional Module MN-GFM 6.7, https://comp.chem.umn.edu/MN-GFM/, Department of Chemistry, Universtity of Minnesota, Minneapolis, MN USA2015.

[76] L. Xing, J.L. Bao, Z. Wang, F. Zhang, D.G. Truhlar, Degradation of Carbonyl Hydroperoxides in the Atmosphere and in Combustion, J. Am. Chem. Soc. 139 (2017) 15821-15835.

[77] E. Pousse, Z.Y. Tian, P.A. Glaude, R. Fournet, F. Battin-Leclerc, A lean methane premixed laminar flame doped with components of diesel fuel part III: Indane and comparison between n-butylbenzene, npropylcyclohexane and indane, Combust. Flame 157 (2010) 1236-1260.

[78] F. Dubnikova, A. Lifshitz, Ring expansion and isomerization in methyl indene and methylene indene radicals. Quantum chemical and transition-state theory calculations, Isr. J. Chem. 43 (2003) 325-338.

[79] S. Fascella, C. Cavallotti, R. Rota, S. Carrà, The Peculiar Kinetics of the Reaction between Acetylene and the Cyclopentadienyl Radical, J. Phys. Chem. A 109 (2005) 7546-7557.

[80] Y.Y. Li, L.D. Zhang, Z.D. Wang, L.L. Ye, J.H. Cai, Z.J. Cheng, F. Qi, Experimental and Kinetic Modeling Study of Tetralin Pyrolysis at Low Pressure, Proc. Combust. Inst. 34 (2013) 1739-1848.

[81] C. Saggese, A. Frassoldati, A. Cuoci, T. Faravelli, E. Ranzi, A wide range kinetic modeling study of pyrolysis and oxidation of benzene, Combust. Flame 160 (2013) 1168-1190.

[82] H. Wang, E. Dames, B. Sirjean, D. A. Sheen, R. Tango, A. Violi, J. Y. W. Lai, F. N. Egolfopoulos, D. F. Davidson, R. K. Hanson, C. T. Bowman, C. K. Law, W. Tsang, N. P. Cernansky, D. L. Miller, R. P. Lindstedt, A high-temperature chemical kinetic model of n-alkane (up to n-dodecane), cyclohexane, and methyl-, ethyl-, n-propyl and n-butyl-cyclohexane oxidation at high temperatures, JetSurF version 2.0, September 19, 2010 (http://web.stanford.edu/group/haiwanglab/JetSurF/JetSurF2.0/index.html).

[83] G. da Silva, J.A. Cole, J.W. Bozzelli, Kinetics of the Cyclopentadienyl + Acetylene, Fulvenallene + H, and 1-Ethynylcyclopentadiene + H Reactions, J. Phys. Chem. A 114 (2010) 2275-2283.

[84] D. Polino, C. Cavallotti, Fulvenallene Decomposition Kinetics, J. Phys. Chem. A 115 (2011) 10281-10289.

[85] C. Cavallotti, M. Derudi, R. Rota, On the mechanism of decomposition of the benzyl radical, Proc. Combust. Inst. 32 (2009) 115-121.

[86] V.V. Kislov, A.M. Mebel, An ab initio G3-type/statistical theory study of the formation of indene in combustion flames. II. The pathways originating from reactions of cyclic C-5 species - Cyclopentadiene and cyclopentadienyl radicals, J. Phys. Chem. A 112 (2008) 700-716.

[87] K. Narayanaswamy, H. Pitsch, P. Pepiot, A chemical mechanism for low to high temperature oxidation of methylcyclohexane as a component of transportation fuel surrogates, Combust. Flame 162 (2015) 1193-1213.

[88] L. Vereecken, J. Peeters, Reactions of chemically activated $\mathrm{C}_{9} \mathrm{H}_{9}$ species II: The reaction of phenyl radicals with allene and cyclopropene, and of benzyl radicals with acetylene, Phys. Chem. Chem. Phys. 5 (2003) 28072817.

[89] H.A. Gueniche, P.A. Glaude, R. Fournet, F. Battin-Leclerc, Rich methane premixed laminar flames doped by light unsaturated hydrocarbons - III. Cyclopentene, Combust. Flame 152 (2008) 245-261.

[90] M.R. Harper, K.M. Van Geem, S.P. Pyl, G.B. Marin, W.H. Green, Comprehensive reaction mechanism for n-butanol pyrolysis and combustion, Combust. Flame 158 (2011) 16-41.

[91] K. Roy, M. Braun-Unkhoff, P. Frank, T. Just, Kinetics of the cyclopentadiene decay and the recombination of cyclopentadienyl radicals with H-atoms: Enthalpy of formation of the cyclopentadienyl radical, Int. J. Chem. Kinet. 33 (2001) 821-833.

[92] H. Richter, J.B. Howard, Formation and consumption of single-ring aromatic hydrocarbons and their precursors in premixed acetylene, ethylene and benzene flames, Phys. Chem. Chem. Phys. 4 (2002) 2038-2055.

[93] B. Heyberger, N. Belmekki, V. Conraud, P.A. Glaude, R. Fournet, F. Battin-Leclerc, Oxidation of small alkenes at high temperature, Int. J. Chem. Kinet. 34 (2002) 666-677.

[94] B. Sirjean, P.A. Glaude, M.F. Ruiz-Lopez, R. Fournet, Theoretical Kinetic Study of Thermal Unimolecular Decomposition of Cyclic Alkyl Radicals, J. Phys. Chem. A 112 (2008) 11598-11610. 
939 [96] A.M. Mebel, Y. Georgievskii, A.W. Jasper, S.J. Klippenstein, Pressure-dependent rate constants for PAH 940 growth: formation of indene and its conversion to naphthalene, Faraday Discuss. 195 (2016) 637-670.

941 [97] H. Richter, S. Granata, W.H. Green, J.B. Howard, Detailed modeling of PAH and soot formation in a 942 laminar premixed benzene/oxygen/argon low-pressure flame, Proc. Combust. Inst. 30 (2005) 1397-1405.

943 [98] Y. Li, J. Cai, L. Zhang, T. Yuan, K. Zhang, F. Qi, Investigation on chemical structures of premixed toluene 944 flames at low pressure, Proc. Combust. Inst. 33 (2011) 593-600.

[99] F. Schulz, M. Commodo, K. Kaiser, G. De Falco, P. Minutolo, G. Meyer, A. D`Anna, L. Gross, Insights into incipient soot formation by atomic force microscopy, Proc. Combust. Inst. 37 (2019) 885-892.

[100] A. Cristadoro, S.U. Kulkarni, W.A. Burgess, E.G. Cervo, H.J. Räder, K. Müllen, D.A. Bruce, M.C. Thies, Structural characterization of the oligomeric constituents of petroleum pitches, Carbon 47 (2009) 2358-2370.

[101] R. Gredel, Y. Carpentier, G. Rouille, M. Steglich, F. Huisken, T. Henning, Abundances of PAHs in the ISM: confronting observations with experimental results, Astron. Astrophys. 530 (2011) 15.

[102] H. Wang, X. You, A.V. Joshi, S.G. Davis, A. Laskin, F. Egolfopoulos, C.K. Law, USC Mech Version II. High-Temperature Combustion Reaction Model of $\mathrm{H}_{2} / \mathrm{CO} / \mathrm{C} 1-\mathrm{C} 4$ Compounds. http://ignis.usc.edu/USC Mech_II.htm.

[103] C.W. Gao, J.W. Allen, W.H. Green, R.H. West, Reaction Mechanism Generator: Automatic construction of chemical kinetic mechanisms, Comput. Phys. Commun. 203 (2016) 212-225.

[104] G.R. Magoon, W.H. Green, Design and implementation of a next-generation software interface for onthe-fly quantum and force field calculations in automated reaction mechanism generation, Comput. Chem. Eng. 52 (2013) 35-45.

959 [105] J.W. Allen, C.F. Goldsmith, W.H. Green, Automatic estimation of pressure-dependent rate coefficients,

[106] A. Cuoci, A. Frassoldati, T. Faravelli, E. Ranzi, OpenSMOKE++: An object-oriented framework for the numerical modeling of reactive systems with detailed kinetic mechanisms, Comput. Phys. Commun. 192 (2015) 237-264.

[107] A. Lifshitz, A. Moran, S. Bidani, Thermal reactions of acetonitrile at high temperatures. Pyrolysis behind reflected shocks, Int. J. Chem. Kinet. 19 (1987) 61-79.

[108] M. Derudi, D. Polino, C. Cavallotti, Toluene and benzyl decomposition mechanisms: elementary reactions and kinetic simulations, Phys. Chem. Chem. Phys. 13 (2011) 21308-21318.

[109] K.O. Johansson, M.F. Campbell, P. Elvati, P.E. Schrader, J. Zádor, N.K. Richards-Henderson, K.R. Wilson, A. Violi, H.A. Michelsen, Photoionization Efficiencies of Five Polycyclic Aromatic Hydrocarbons, J. Phys. Chem. A 121 (2017) 4447-4454.

[110] B. Shukla, M. Koshi, A highly efficient growth mechanism of polycyclic aromatic hydrocarbons, Phys. Chem. Chem. Phys. 12 (2010) 2427-2437.

[111] B. Shukla, M. Koshi, A novel route for PAH growth in HACA based mechanisms, Combust. Flame 159 (2012) 3589-3596.

[112] T. Miersch, H. Czech, B. Stengel, G. Abbaszade, J. Orasche, M. Sklorz, T. Streibel, R. Zimmermann, Composition of carbonaceous fine particulate emissions of a flexible fuel DISI engine under high velocity and municipal conditions, Fuel 236 (2019) 1465-1473.

[113] H. Lim, T.M. Ahmed, C. Bergvall, R. Westerholm, Automated clean-up, separation and detection of polycyclic aromatic hydrocarbons in particulate matter extracts using a 2D-LC/2D-GC system: a method translation from two FIDs to two MS detectors, Anal. Bioanal. Chem. 409 (2017) 5619-5629.

[114] Y. Li, L. Zhang, T. Yuan, K. Zhang, J. Yang, B. Yang, F. Qi, C.K. Law, Investigation on fuel-rich premixed flames of monocyclic aromatic hydrocarbons: Part I. Intermediate identification and mass spectrometric analysis, Combust. Flame 157 (2010) 143-154. 
984 [115] C. Cavallotti, D. Polino, A. Frassoldati, E. Ranzi, Analysis of Some Reaction Pathways Active during 985 Cyclopentadiene Pyrolysis, J. Phys. Chem. A 116 (2012) 3313-3324.

986 [116] J.D. Savee, T.M. Selby, O. Welz, C.A. Taatjes, D.L. Osborn, Time- and Isomer-Resolved Measurements 987 of Sequential Addition of Acetylene to the Propargyl Radical, The Journal of Physical Chemistry Letters 6 $988 \quad$ (2015) 4153-4158.

989 [117] D. Trogolo, A. Maranzana, G. Ghigo, G. Tonachini, First Ring Formation by Radical Addition of 990 Propargyl to But-1-ene-3-yne in Combustion. Theoretical Study of the C7H7 Radical System, J. Phys. Chem. 991 A $118(2014) 427-440$.

992 [118] P.D.S. N., K.R. I., K. Oleg, A. Musahid, Selective Formation of Indene through the Reaction of Benzyl 993 Radicals with Acetylene, ChemPhysChem 16 (2015) 2091-2093.

994 [119] N.J. Reilly, G. da Silva, C.M. Wilcox, Z. Ge, D.L. Kokkin, T.P. Troy, K. Nauta, S.H. Kable, M.C. 995 McCarthy, T.W. Schmidt, Interconversion of Methyltropyl and Xylyl Radicals: A Pathway Unavailable to the 996 Benzyl-Tropyl Rearrangement, J. Phys. Chem. A 122 (2018) 1261-1269.

997 [120] R. Sivaramakrishnan, R.S. Tranter, K. Brezinsky, High pressure pyrolysis of toluene. 2. Modeling benzyl 998 decomposition and formation of soot precursors, J. Phys. Chem. A 110 (2006) 9400-9404.

999 [121] M. Verdicchio, B. Sirjean, L.S. Tran, P.-A. Glaude, F. Battin-Leclerc, Unimolecular decomposition of 000 tetrahydrofuran: Carbene vs. diradical pathways, Proc. Combust. Inst. 35 (2015) 533-541.

001 [122] K.P. Somers, J.M. Simmie, F. Gillespie, C. Conroy, G. Black, W.K. Metcalfe, F. Battin-Leclerc, P. 002 Dirrenberger, O. Herbinet, P.-A. Glaude, P. Dagaut, C. Togbé, K. Yasunaga, R.X. Fernandes, C. Lee, R. Tripathi, 003 H.J. Curran, A comprehensive experimental and detailed chemical kinetic modelling study of 2,5-dimethylfuran 004 pyrolysis and oxidation, Combust. Flame 160 (2013) 2291-2318.

.005 


\section{Figure Captions}

.008

\section{(Color figures in electronic version only)}

Scheme 1 (a) C-H and (b) C-C bond energies of indene, calculated at $298 \mathrm{~K}$. Unit: kcal/mol.

Scheme 2 Reaction scheme proposed for $\mathrm{C}_{9} \mathrm{H}_{7}+\mathrm{C}_{3} \mathrm{H}_{3}$. Solid arrows represent elementary reaction steps. Blue dashed arrow highlights the key reaction step.

Scheme 3 Reaction scheme proposed for $\mathrm{C}_{9} \mathrm{H}_{8}+\mathrm{C}_{5} \mathrm{H}_{5}$ and $\mathrm{C}_{9} \mathrm{H}_{7}+\mathrm{C}_{5} \mathrm{H}_{6}$. Solid arrows represent elementary reaction steps; dashed arrows represent reactions with multiple steps.

Scheme 4 Reaction scheme proposed for $\mathrm{C}_{9} \mathrm{H}_{7}+\mathrm{C}_{6} \mathrm{H}_{5}$. Solid arrows represent elementary reaction steps; dashed arrows represent reactions with multiple steps.

Scheme 5 Reaction schemes (a) for $\mathrm{C}_{5} \mathrm{H}_{6}+\mathrm{C}_{5} \mathrm{H}_{5}$ calculated by Kislov et al. [86], (b) proposed here for $\mathrm{C}_{9} \mathrm{H}_{8}+\mathrm{C}_{9} \mathrm{H}_{7}$. Solid arrows represent elementary reaction steps; broad arrows represent reactions with multiple steps.

Scheme 6 Reaction schemes (a) for $\mathrm{C}_{5} \mathrm{H}_{5}+\mathrm{C}_{5} \mathrm{H}_{5}$, (b) proposed for $\mathrm{C}_{9} \mathrm{H}_{7}+\mathrm{C}_{9} \mathrm{H}_{7}$. Solid arrows represent elementary reaction steps; dash arrows represent reactions with multiple steps.

Figure 1 Measured mole fraction profiles of indene $\left(\mathrm{C}_{9} \mathrm{H}_{8}\right)$ and its main decomposition products in a flow reactor at 30 (black squares) and 760 (red circles) Torr. Shadows with the data plots represent the uncertainty of the measurements. Lines are predictions of the current model.

Figure 2 Mole fraction profiles of small intermediates measured in indene pyrolysis in a flow reactor at 30 (black squares) and 760 (red circles) Torr. Shadows with the data plots represent the uncertainty of the measurements. Lines are the predictions of the present model. Experimental data and simulations of species with small concentration are scaled with a common factor.

Figure 3 Mole fraction profiles of dominant (common) PAHs measured in indene pyrolysis in a flow reactor at 30 (black squares) and 760 (red circles) Torr. Shadows with the data plots represent the uncertainty of the measurements. Lines indicate the model predictions.

Figure 4 Mole fraction profiles of uncommon PAHs measured in indene pyrolysis in a flow reactor at 30 (black squares) and 760 (red circles) Torr. Shadows with the data plots represent the uncertainty of the measurements. Lines indicate the model predictions.

Figure 5 Reaction flux analysis of indene decomposition at 17.5\% conversion. Arrows indicate reaction steps; the numbers indicate the conversion ratios of each step at 30 (black) and 760 torr (red). 
038 Figure 6 Sensitivity of R1 (a) and R2 (b) to the consumption of indene at 30 and 760 Torr. The dash lines and dotted lines are the simulation results of indene using the rate coefficients of R1 and R2 multiplied or divided by a factor of three.

041 Figure 7 Main PAH formation pathways according to the present model: (a) indenyl reactions to form $\mathrm{C}_{10}-\mathrm{C}_{12}$ species, (b) indenyl reactions to form $\mathrm{C}_{17}$ and $\mathrm{C}_{18}$ species. The blue frame highlights the larger RSR generated by indenyl radical.

Figure 8 Main PAH formation pathways according to the present model from indenyl to $\mathrm{C}_{13}-\mathrm{C}_{16}$ species. The blue frames highlight the larger RSRs generated by indenyl radical.

Figure 9 Modeling results of indene decomposition in a shock tube; experimental data taken from [62]. Simulated results were shifted by $80 \mathrm{~K}$ according to the literature $[121,122]$.

Figure 10 Predicted mole fraction profiles of the species measured in shock tube indene pyrolysis 050 [62]. Simulated results were shifted by $80 \mathrm{~K}$ according to the literature $[121,122]$. 


\section{Supplementary Materials}

053 SMM1. Temperature profiles measured along the flow reactor.xlsx

054 SMM2. Experimental details.docx

055 SMM3. Quantum calculation.docx 